\title{
Quantitative Perfusion \\ Measurements in a Novel \\ Large Animal Stroke Model
}

by

Alexandra Ereni King

\author{
A Thesis \\ submitted to Victoria University of Wellington \\ in fulfilment of the \\ requirements for the degree of \\ Master of Science \\ in Physics.
}

Victoria University of Wellington

2021 



\begin{abstract}
Stroke is a leading cause of death worldwide [1], and is the third leading cause of death and the leading cause of serious adult disability in New Zealand [2]. The aim of this project was to quantify perfusion changes in the brains of 20 sheep that underwent a novel surgical model of transient ischemic stroke.

The sheep, with its large, gyrencephalic brain, presents a promising, potential animal model for stroke that could help to bridge the historical gap in translational research in stroke therapies [3]. However, we require that an animal model can replicate human patterns of disease in order for it to be a meaningful model for research into potential stroke therapies for humans. It was this replication of human patterns of disease, in terms of perfusion, that was under investigation in this project.

Dynamic Contrast Enhanced (DCE) MRI images were obtained from each animal before stroke, and at 24 hours, 3 days, 6 days, and 28 days post-stroke. It was found that perfusion from the DCE-MRI series was quantifiable using the extended Tofts model in the form of the parameters $K_{\text {trans }}, v_{\mathrm{e}}$ and $v_{\mathrm{p}}$. The parameter values calculated from this project reflect known human patterns of disease in terms of global $K_{\text {trans }}$ changes in the affected hemisphere [4], which were found to increase by more than $60 \%$ in the stroke hemisphere, reflecting the increased permeability following blood brain barrier breakdown. In manually selected regions of cytotoxic and vasogenic edema, it was found that the estimated parameters in these regions reflected known perfusion changes in these types of edema in humans [5]. Finally, the peak post-stroke permeability time point, as determined by $K_{\text {trans }}$, was found to align exactly with when we would expect vasogenic edema, a type of cerebral swelling that causes increased barrier permeability, to dominate in humans [5].
\end{abstract}


This thesis is the first time these DCE-MRI datasets have been analysed, and there remains a wealth of physiological and MRI data available for this animal cohort. Avenues for future research include investigation into perfusion-diffusion mismatch in this animal model, further consideration of individual animal characteristics in analysis, and use of these results as a point of comparison for future research into pharmaceutical agents for treatment of stroke, and in new non-contrast perfusion measurement techniques. 


\section{Acknowledgements}

I would first like to thank my supervisors, Sergei Obruchkov, Shieak Tzeng and Paul Teal for their support, kindness and insights throughout this thesis.

A special thanks to Annabel Sorby-Adams for her invaluable contributions to this thesis, her wealth of ovine physiological knowledge, and generosity with her time even with a 12 hour time difference. Thanks also to Renée Turner and her team at the University of Adelaide, without whose tireless work the data under analysis in this thesis would not exist.

I'd like to thank everyone at the NMR lab at Victoria University of Wellington, and Dion Thomas particularly for converting my code to work on the university's supercomputer. The lab was the best place I could have hoped to undertake my Masters degree, and I remain grateful for everyone's warmth, friendship and support.

I would like to thank Sunthara Rajan Perumal for the acquisition of the MRI and to acknowledge the facilities and technical assistance of the National Imaging Facility, a National Collaborative Research Infrastructure Strategy (NCRIS) capability, at LARIF, SAHMRI. I would also like to acknowledge Nawaf Yassi and Daniel Zappia for optimising the scan and sequence protocols used in the animal experiments, and Aoife Reid for insights on the pulse sequences used in these experiments.

I would like to thank and acknowledge Ash King for designing the physiological diagrams throughout Chapter 2. Personal thanks to my partner, my sisters, and my friends for supporting me through all the ups and downs of the past year. 


\section{Contents}

1 Introduction 1

2 Background Information 3

2.1 Stroke and Perfusion . . . . . . . . . . . . . . 3

2.1.1 Physiology of Perfusion . . . . . . . . . . . . 4

2.1.2 Stroke Background . . . . . . . . . . . . 5

2.1.3 Stroke Disease Mechanism . . . . . . . . . . . . 6

2.1.4 Stroke Therapy Literature Review . . . . . . . . . . . 8

2.2 Physics of MRI . . . . . . . . . . . . . . . . . . . . . . 11

$2.2 .1 \quad$ NMR . . . . . . . . . . . . . . . . 11

2.2.2 MRI Pulse Sequences . . . . . . . . . . . . . . 15

2.3 DCE-MRI Acquisition and Qualitative Analysis . . . . . . . . 17

2.3.1 DCE-MRI Acquisition . . . . . . . . . . . . 18

2.3.2 Qualitative and semi-quantitative DCE-MRI analysis . 20

2.4 Quantitative DCE-MRI Analysis . . . . . . . . . . . 23

2.4.1 General Introduction to Tracer Kinetics . . . . . . . . 23

2.4.2 Pharmacokinetic Models of Perfusion . . . . . . . . 26

2.5 DCE-MRI in Stroke Literature Review . . . . . . . . . . . . 30

3 Animal Experiments 33

3.1 Surgical procedure . . . . . . . . . . . . . . . . 33

3.2 Image Acquisition . . . . . . . . . . . . . . . . . . . . . . 34 
4 DCE-MRI Image Processing Methods 37

4.1 Overview of Image Processing Tools . . . . . . . . . . . . . . . 38

$4.2 \quad B_{1}$ Field Inhomogeneity Consideration . . . . . . . . . . . . . 40

4.2.1 $B_{1}$ field inhomogeneities in a second animal cohort . . 41

4.3 T1 Map Generation . . . . . . . . . . . . . . . . . . . . 42

4.3.1 Algebraic $T_{1}$ determination . . . . . . . . . . . . . . 42

4.3.2 Verification of $T_{1}$ map values . . . . . . . . . . . . 43

4.4 Signal to Concentration Conversion . . . . . . . . . . . 45

4.4.1 DCE signal to concentration conversion . . . . . . . . 46

4.4.2 Determination of $R_{\mathrm{Gd}} \ldots \ldots \ldots \ldots$. . . . . . 47

4.5 Pharmacokinetic Model Selection . . . . . . . . . . . . . 48

4.5.1 Statistical Considerations in PK model selection . . . . 49

4.5.2 Temporal Resolution in PK model selection . . . . . . 51

4.5.3 Discussion of PK model selection . . . . . . . . . . . 53

4.6 Development of Curve fitting Techniques . . . . . . . . . . 54

4.6.1 Curve Fitting Algorithm . . . . . . . . . . . . . 54

4.6 .2 Initial Guess . . . . . . . . . . . . . . . . . 54

4.6.3 Treatment of Noise . . . . . . . . . . . . 56

4.6.4 Computational Considerations in Fitting . . . . . . 58

4.6.5 Synthetic data verification of Curve Fitting Techniques 59

4.7 Discussion of DCE-MRI Analysis Experimental Methods . . . 62

4.7.1 Discussion of $T_{1}$ map generation . . . . . . . . . . 62

4.7.2 Discussion of Curve Fitting Techniques . . . . . . . . 63

4.7.3 Exploration of existing software packages . . . . . . . 66

4.7.4 Summary of DCE-MRI Analysis Methods . . . . . . 67

5 AIF Selection Methods $\quad 69$

5.1 AIF Selection Algorithm . . . . . . . . . . . . . . . 70

5.1.1 Desirable AIF Characteristics . . . . . . . . . . 70

5.1 .2 AIF Search Criteria . . . . . . . . . . . 71

5.2 Anatomy Based AIF Selection . . . . . . . . . . . . . . 72

5.2.1 Anatomical Masks . . . . . . . . . . . . . 72 
5.2.2 Three Test Anatomy Based Masks . . . . . . . . . . . 74

5.2 .33 Test AIF Mask Results . . . . . . . . . . . . . . . . 76

5.3 Physiological Validation of AIFs . . . . . . . . . . . . 78

5.3.1 Preparation for further analysis . . . . . . . . . 79

5.4 Discussion of AIF selection methods . . . . . . . . . . . . . . . 80

5.4.1 Discussion of AIF selection algorithm . . . . . . . . . . 80

5.4.2 AIF Selection Improvements for Future Research . . . 82

5.4.3 Summary of AIF selection methods . . . . . . . . . . 84

6 Results and Analysis $\quad 85$

6.1 Perfusion Maps . . . . . . . . . . . . . . . . . . . . 86

6.2 Hemisphere Level Analysis . . . . . . . . . . . . . . . . . . . . 86

6.2.1 AIF Based Exclusion . . . . . . . . . . . . . . 88

6.2.2 Error in Fit Based Exclusion . . . . . . . . . . . . . . . 91

6.2.3 Histogram Level Analysis . . . . . . . . . . . . . . . . 95

6.2.4 Summary of Hemisphere Level Analysis . . . . . . . . . 98

6.3 Small ROI Level Analysis . . . . . . . . . . . . . . . . . . . . 99

6.3.1 Consideration of ischemic tissue heterogeneity . . . . . 99

6.3.2 Small ROI Generation . . . . . . . . . . . . . . . 102

6.3.3 Vasogenic, cytotoxic and healthy tissue results. . . . . 102

7 Conclusion and Future Research $\quad 107$

7.1 Conclusions . . . . . . . . . . . . . . . . . . . . 107

7.2 Future Research . . . . . . . . . . . . . . . . . . . . . 109

7.2.1 Animal characteristic based analysis . . . . . . . . 109

7.2 .2 Summary . . . . . . . . . . . . . . . . . 110

8 Appendices $\quad 111$

8.1 Two compartment exchange model impulse response function . 111

8.2 Table of Abbreviations . . . . . . . . . . . . . . . . . . . . . . 112

8.3 Infarct Size Table . . . . . . . . . . . . . . . . . . . . . 113

8.4 CDF Analysis . . . . . . . . . . . . . . . . . . . . 114 


\section{Chapter 1}

\section{Introduction}

The aim of this project was to quantify the perfusion changes in the brains of 20 sheep.

These animals each underwent a novel surgical procedure that simulated a stroke, and the sheep themselves were metaphorical guinea pigs for this model of stroke and how well it can replicate the myriad of physiological complexities that occur post-stroke in humans.

Replication of human patterns of disease is the primary desirable characteristic for any animal model in translational research. While rodents are frequently used in all sorts of behavioural and physiological research designed for future application to humans, the post-stroke human brain is a highly complex, closed system in terms of pressure and perfusion changes. As such, the sheep's large, gyrencephalic brain, susceptible to intracranial pressure changes and fed by a complex series of arteries presents a potential animal model for stroke that could help to bridge the gap in translational research in stroke therapies.

However, in order to prove that the anatomical features that appear to lend support to the use of this animal model do in fact result in patterns of disease similar to that in humans, we need to show that what happens in humans can happen in sheep. That is, known post-stroke patterns of disease can be replicated in this animal model. Without this, the use of this animal 
model in testing of pharmaceutical agents designed for human application would be worthless.

This project specifically aims to quantify the perfusion changes seen in the sheep's brain at the time points for which DCE-MRI images were acquired for this animal cohort: prestroke, 24 hours, 3 days, 6 days and 28 days post-stroke. By quantifying these changes we can

1. determine quantitative, measurable perfusion parameters directly from the affected ischemic tissue in each animal,

2. measure changes in perfusion in the sheep's brain and compare to known post-stroke perfusion changes in humans for validation of this animal model, and

3. provide a quantitative foundation for future research using this animal model.

Relevant background information including details on the physiology of stroke, the physics of NMR and the particular MRI technique under analysis in this project are given in Chapter 2. The following Chapters detail the experimental methods used in the animal experiments, divided into methods used in DCE-MRI analysis and in AIF selection. Chapter 6 presents results, progressing through hemisphere level analysis down to small ROI level analysis to isolate perfusion changes in both cytotoxic and vasogenic edema. Results from small ROI analysis demonstrated that the perfusion changes associated with vasogenic edema formation occur at the exact time point we would expect to see according to the human pattern of disease. Finally, conclusions and avenues for future research are given in Chapter 7 . 


\section{Chapter 2}

\section{Background Information}

The main problem under investigation in this thesis can be written simply as:

How does each animal's cerebral perfusion change over time as a result of the induced stroke?

When written this way, the problem could be interpreted purely physiologically. However in this project we wish to approach it quantitatively, through modelling, fitting and image analysis, and drawing on knowledge from several areas of physics, including nuclear magnetic resonance, magnetic resonance imaging, image processing, and multi-compartmental pharmacokinetic models of perfusion.

Relevant background information is presented in this chapter. The first two sections introduce important concepts in stroke, physiology and perfusion, and NMR and MRI physics respectively. The third Section onwards then draws on information from both of these areas and introduces DCE-MRI analysis techniques, and a literature review of past stroke research utilising DCE-MRI.

\subsection{Stroke and Perfusion}

The aim of this Section is to give an overview of basic physiology pertaining to perfusion and the mechanism of disease of stroke. 


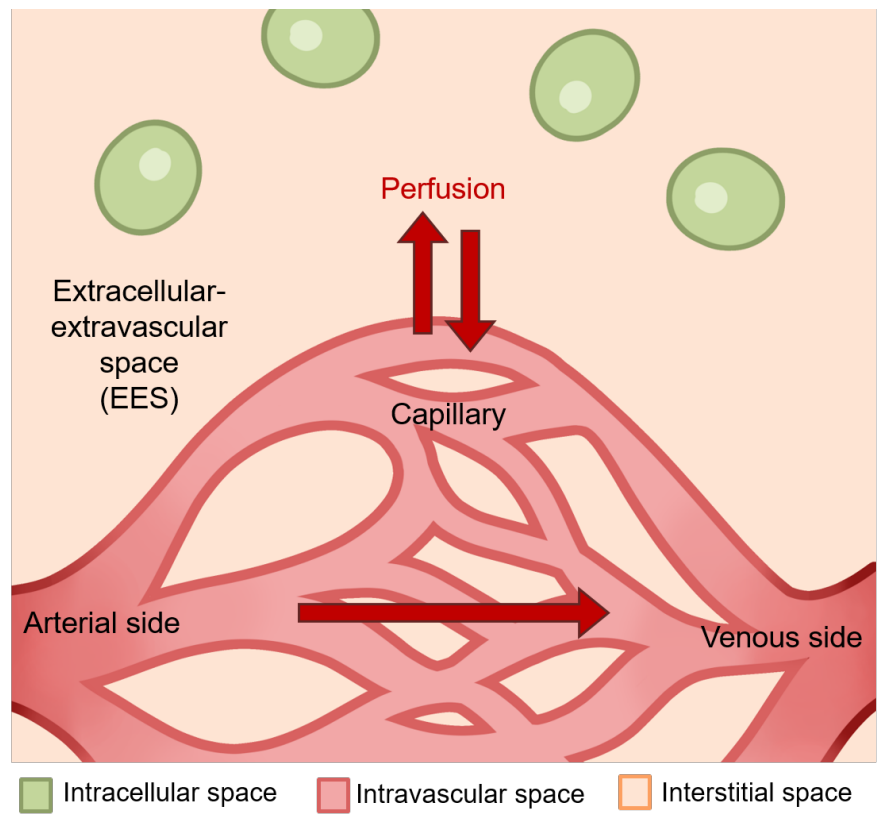

Figure 2.1: Diagram of perfusion at the capillary level. Oxygen and nutrients carried by the blood are delivered to tissue while waste products are carried away.

\subsubsection{Physiology of Perfusion}

\section{Perfusion}

Perfusion takes place everywhere in the body where there is a blood supply, from cortical bone [6] to the liver [7], and is a process of critical importance for all bodily functions. The cerebrovascular system is made up of a system of arteries, capillaries and veins that transport blood to and from the brain.

The arteries feeding the brain can be divided into the posterior cerebral arteries, anterior cerebral arteries, and internal carotid artery which bifurcates into the middle cerebral artery and posterior communicating artery. This network of arteries is linked by the posterior and anterior communicating arteries which form a ring at the base of the skull called the circle of Willis [8].

The capillaries are characterised by their structure of a single outer layer of 
endothelial cells, which allows for the passage of blood into the extravascular space in order to deliver oxygen and essential nutrients to a tissue. The process by which blood passes through the capillary bed within tissue is called perfusion. This process is important as it regulates the flow of ions, oxygen, and waste products across the capillary walls.

\section{Cerebral Perfusion}

Cerebral perfusion refers to the blood supplied to the brain tissue parenchyma via a series of blood vessels making up the cerebral circulatory system. Perfusion within the brain differs from that in the rest of the body due to the specialised structure of the capillary walls, which form a thicker, less permeable membrane called the blood-brain barrier (BBB) [9]. This specialised structure is specially adapted for capillaries in the brain due to the increased sensitivity of neurons to oxygen deprivation due to their high metabolic rate [9]. As a result, the brain is highly susceptible to irreversible cell damage arising from changes in blood supply.

\subsubsection{Stroke Background}

A stroke is the sudden onset of a neurological deficit, such as localised loss of sensation, as a result of disruption of blood flow to part of the brain. As outlined in Section 2.1.1, the brain is particularly susceptible to cell damage and death arising from blood supply change due to the high metabolic rate of neurons [9]. As such, within a very short amount of time from symptom onset, stroke can have devastating and irreversible consequences.

Stroke is a leading cause of death and disability worldwide [1]. In New Zealand, stroke is the third leading cause of death and the leading cause of serious adult disability [2]. It is estimated that hospitalisation, rehabilitation, burden on family and caregivers, and the increased disability risk and early mortality associated with stroke costs New Zealand approximately $\$ 1$ billion NZD a year. Due to the ageing population, the number of people experiencing 
stroke in New Zealand is predicted to increase by $40 \%$ in the next 10 years, increasing this figure to $\$ 1.7$ billion NZD by 2038 [10]. Aside from the economic cost, the cost to quality of life, and the emotional and physical toll on the affected and their family members can be immense. Just over half of stroke survivors will regain functional independence, but the majority will not return to their prestroke level of functioning [11]. Measures of recovery post-stroke, and even the definition of "stroke recovery", remains ambiguous, and as such measurements of patient outcomes following stroke can vary greatly depending on how this is defined [11]. In some contexts, recovery will imply a complete recovery of prestroke functionality, whereas in other cases recovery may mean only the improvement in some measure of stroke related impairment [12]. There exist many clinical scales for assessment of such impairments, such as the modified Rankin score, Barthel index or the NIH Health Stroke Scale [11]. Each of these scales use practical measurements such as the patient's ability to walk, dress, use the stairs or answer questions correctly [11].

\subsubsection{Stroke Disease Mechanism}

Ischemic stroke is caused by the blockage of a cerebral blood vessel. This blockage may be either a dislodged bolus such as a blood clot, as in embolic stroke, or more commonly, the atherosclerotic build up in the artery [13]. The blockage, if sustained, can lead to cerebral infarction and cause what is commonly known as an ischaemic stroke. Epidemiologically, ischemic strokes are the most common type of stroke $(87 \%$ [1]) and the type of stroke that will be the focus of this project.

The blockage of an artery feeding the brain can lead to many complex, interacting effects, one of the most dangerous of which involves the swelling, and subsequent increase in intracranial pressure in the affected hemisphere.

In humans, the intracranial contents are incompressible, and the parenchyma is in a state of equilibrium with the blood and cerebrospinal fluid [14]. Changes in intracranial pressure in turn affect cerebral perfusion pressure and the 
(a)

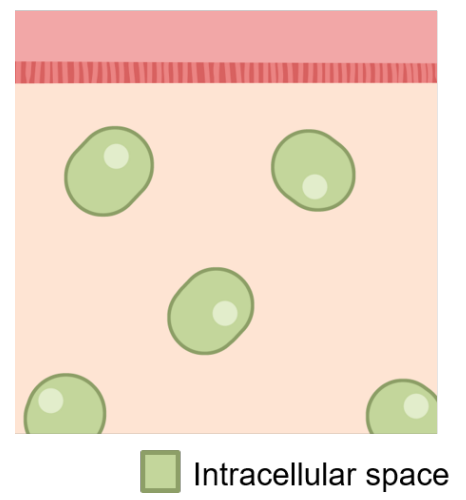

(b)

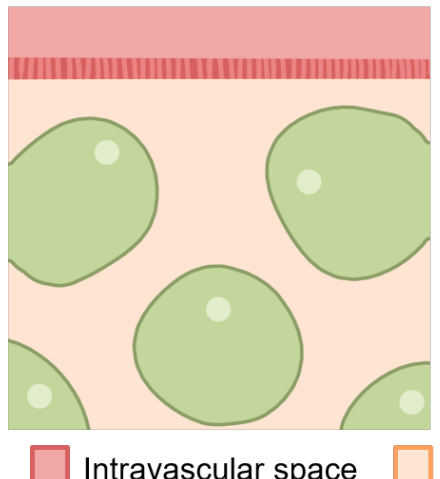

(c)

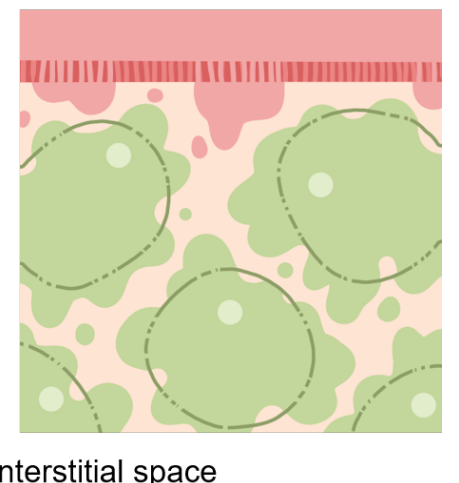

Figure 2.2: Time progression of (a) healthy tissue (b) cytotoxic edema at 24 hours post-stroke and (c) vasogenic edema between 3 and 5 days post-stroke.

normally carefully governed equilibrium of fluid exchange throughout the brain can be quickly compromised.

Following a middle cerebral artery (MCA) occlusion, an area of the brain will have been deprived of oxygen from the blood for a period of time. This tissue will be damaged following oxygen starvation, due to the high metabolic demands of cells in the brain. This damage has many dangerous effects, including the breakdown of the endothelial cells forming the blood brain barrier, leading to further fluid build up and a vicious cycle of intracranial hypertension and compromised cerebral perfusion, and cell injury [15].

MCA infarction occurs in $10 \%$ of ischemic stroke patients but is associated with a mortality rate of up to $80 \%$ as a result of this dangerous cycle [16]. Cerebral edema also accounts for $5 \%$ of all deaths in patients with cerebral infarction [17].

This build up of fluid is called cerebral edema, or swelling, and is known to significantly impact the degree of neurological damage that will be sustained by the patient [18]. The two main types of edema associated with ischemic stroke are cytotoxic and vasogenic edema [19]. Cytotoxic edema occurs up to 
24 hours following stroke as cell membrane pumps fail and cells accumulate fluid within the cell membrane, causing them to swell and have restricted diffusion. Vasogenic edema follows between 3 and 5 days post-stroke and involves the breakdown of the cell membranes and the blood brain barrier, causing water and plasma from the intravascular space to leak into the interstitial space [5].

\section{Clinically Relevant Timepoints}

Clinically relevant post-stroke timepoints have been defined to categorise typical onset times of post-stroke complications. These are the hyperacute phase, up to 24 hours post-stroke, the acute phase, 1-7 days post-stroke, the subacute phase, one week to six month post-stroke, and finally the chronic phase, post six months [20].

The above definitions are taken from the Stroke Recovery and Rehabilitation Roundtable, but there remains much variation in the definition of each of the phases, particularly in the cutoff point between the subacute and chronic phase.

\subsubsection{Stroke Therapy Literature Review}

Pharmacological agents investigated as potential stroke treatments range from thrombolytics, designed to dissolve a clot causing occlusion, to neuroprotectants, designed to minimise damage to healthy cells following ischemia. Over 1000 such drugs have been considered in preclinical trials, however only one has been approved for routine use in stroke patients; a thrombolytic agent called tissue plasminogen activator (tPa) [16][21]. This poor translation from experimental setting to clinical trials is the subject of debate in many recent publications, and has been attributed to factors such as the choice of animal model [22], the drugs selected to progress from experimental setting to clinical trial [21] and study design and publication bias in neuroprotective publications [23]. 


\section{Challenges of Using Animal Models in Stroke Research}

A roundtable was formed in 1999 to address the translational failure from preclinical studies to clinical trials, and to provide recommendations to improve the quality of preclinical research into potential stroke therapies. The Stroke Therapy Academic Industry Roundtable has gone on to publish recommendations periodically, the most recent of which includes revisions in the context of the latest developments in thrombectomy technology [3].

Previous STAIR recommendations pertaining to animal models in preclinical research remained unchanged in the 2019 publication, and included guidelines such as recommending the use of both male and female animals, investigation in at least two independent laboratories, and appropriate use of randomisation and blinding [3].

\section{Small Animal Models}

Small animal models are generally more abundant, cost-effective, and are associated with fewer ethical restrictions than larger animal models. Rodent models are the most commonly used animal model in preclinical stroke research for these reasons, and the rabbit model remains the only animal model that has successfully yielded a treatment approved for use in stroke patients; tissue plasminogen activator (tPa) [24].

The use of rodent models has many advantages over other models, such as their relative cost effectiveness and availability compared to larger animal models, their genetic homogeneity and fewer public welfare concerns associated with testing on rats and mice [25]. However, the rodent's brain differs structurally and functionally from the human's due to its relative lack of white matter [26]. Like other small animal models such as the rabbit, the rodent's brain is lissencephalic, or smooth, lacking the sulci and gyri found in larger animals and humans [26]. Consequently, differences in mortality rates and intracranial pressure (ICP) changes following middle cerebral artery occlusion (MCAO) have been observed in studies of rodent models [27] as well 
as high rates of spontaneous reperfusion ( $80 \%$ within 3 hours post MCAO), contrary to humans [28][3]. These differences are a result of the differing structure of the rodent brain, such as its comparatively weak tentorium cerebelli, the superior covering of the cerebellum, which allows for reduction in ICP following ischemic event [29]. This may be a significant difference due to the high mortality rate in humans associated with ICP increases.

As such, the rodent brain's ability to counter ICP increases following MCA infarction does not align with known human patterns of brain injury. It has been argued that the differences in neuroanatomy, mechanism of injury and post MCAO pattern of damage in rodents could be a cause of the poor translation human clinical trials [22].

\section{Large Animal Models}

Large animal models such as sheep and non-human primates possess gyrencephalic brains, more comparable in size and structure to the human's than small animal models [25]. Animal models such as the sheep or pig also have a more comparable white to grey matter ratio and cerebrovascular diameter, better approximating the anatomy of the human brain [22].

It has been shown that the ovine brain demonstrates increased ICP following an MCA occlusion, even following the loss of cerebrospinal fluid (CSF) during surgery [27], which is closer to the human pattern of damage following ischemic stroke than the rodent's more adaptable brain. Larger animal models have also been demonstrated to allow for easier physiological monitoring following surgery than smaller animal models [25].

However, while larger animals' gyrencephalic brains are closer to the anatomy of the human brain, providing opportunity for increased translational ability, the surgical procedures required in order to induce a simulated ischemic event are often more complex, labour intensive and time consuming than those required for rodent models [22]. Additionally, the maintenance and monitoring of the animals during the procedure is financially costly and labour intensive, often involving prolonged periods under anaesthesia [25]. 


\section{Ongoing areas of debate}

Aside from the outlined differences in neuroanatomical structure, a fundamental issue with the use of animal models remains: animal models simulate the stroke event, not the underlying pathology that would have given rise to the stroke in a human [26]. It can also be challenging to quantify the neurological deficit following ischemic stroke in the animals, and a variety of tests have been investigated for various animal models [26]. In experiments requiring anaesthesia, the effect of the anaesthetic agent on results also remains an area of uncertainty [22]. Despite the ongoing debates and areas of uncertainty in preclinical stroke research involving animal models, the use of such models has led to the important discoveries of critical cerebral blood flow thresholds for irreversible cell death, mechanisms of injury in the minutes, hours and days following MCAO, and the development of models of permanent and transient MCAO [30].

\section{$2.2 \quad$ Physics of MRI}

Magnetic resonance imaging takes advantage of differences in $T_{1}$ and $T_{2}$ within tissues to provide contrast in images. These differences can be accentuated by the sequence of pulses applied to the sample, and the setting of two acquisition parameters: $T_{E}$ and $T_{R}$, the echo time and repetition time respectively. The basic physics of NMR is presented in Section 2.2.1, and pulse sequences and acquisition methods relevant to this project are covered in the following sections.

\subsubsection{NMR}

Nuclear magnetic resonance refers to manipulation of intrinsic properties of nuclei in a magnetic field in order to gather information about those nuclei.

The property that is manipulated in NMR is called spin, which can be considered to give the nucleus a magnetic moment and an angular momentum. 
Due to the magnetic moment property of spin, a proportion of spins in a magnetic field will align with the applied magnetic field, $B_{0}$. Due to the angular momentum property of spin, in the situation described above, the spins precess about the direction of $B_{0}$.

In NMR we are interested in the net magnetisation of these spins rather than individual spins, and so we introduce the concept of the magnetisation vector, $\mathbf{M}$, to describe the direction of the net magnetisation of a sample.

In the case of proton NMR, the target nucleus is hydrogen, or a single proton, which is perturbed with a radio frequency pulse equal to that of the resonant precessional frequency of the nucleus. This frequency is defined by the Larmor equation to be

$$
\omega_{0}=\gamma \mathrm{B}_{0}
$$

In this equation, $\omega_{0}$ is the precessional frequency of the nucleus, in rad $\sec ^{-1}, \gamma$ is the gyromagnetic ratio of the nucleus- which for protons is $2.675 \times$ $10^{-8} \mathrm{rads}^{-1} \mathrm{~T}^{-1}$, and $B_{0}$ is the magnetic field strength. If we set the frequency of a pulse to be approximately equal to that of the nucleus' precessional frequency, we can detect nuclear spin excitations following application of the pulse. Depending on the pulse length, the angle of this excitation may be manipulated, and intrinsic properties of the nucleus can be determined from the relaxation following excitation.

\section{Free induction decay}

Free induction decay refers to the signal detected following spin excitation due to application of a radio frequency pulse of frequency equal to or close to the Larmor frequency of a nucleus. This phenomenon is illustrated in Figure 2.3 .

Following spin excitation the magnetisation vector precesses about the $\mathrm{z}$ axis and the component of magnetisation in the transverse plane is a decaying sinusoidal curve. This changing magnetic field in the transverse plane will, according to Faraday's law of induction, induce a voltage change in a coil 


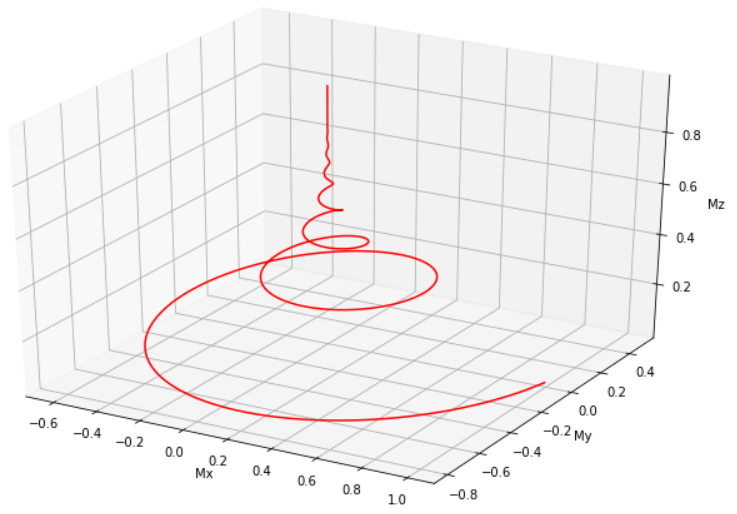

(a)
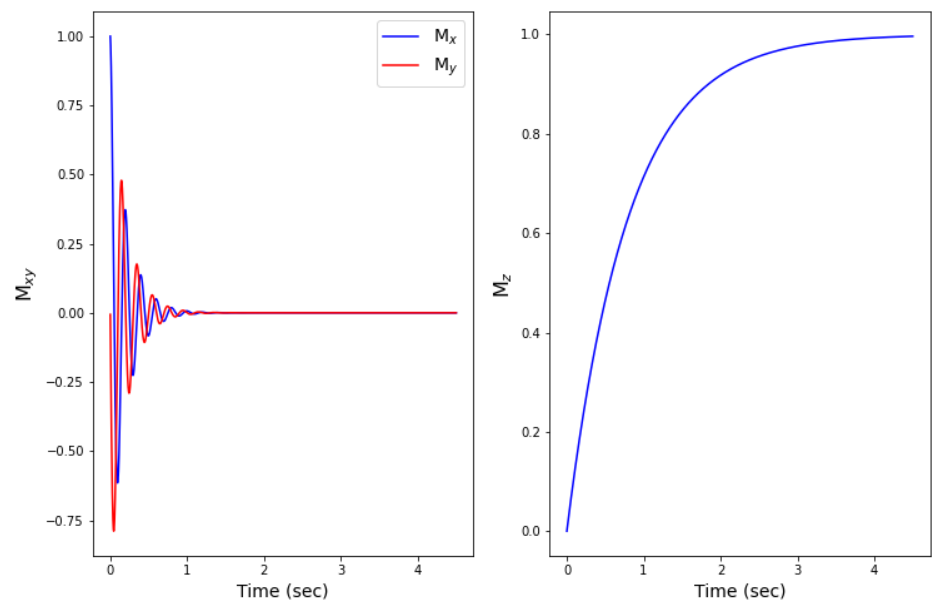

(b)

Figure 2.3: (a) Diagram of relaxation of magnetisation vector following application of a 90 degree pulse (b) Free Induction Decay measured in transverse plane (c) relaxation of longitudinal component of magnetisation, $M_{\mathrm{z}}$ 
that can be placed near the sample, and the decay signal can be observed as an FID curve.

In this example, the pulse applied is a 90 degree pulse, meaning that all initial magnetisation in the longitudinal direction is flipped into the transverse plane. The flip angle can be manipulated by adjusting characteristics of the applied RF pulse and is governed by

$$
\theta=\gamma \int_{0}^{t} B_{1}(t) d t
$$

In the case of an ideal hard pulse, the flip angle induced in the magnetisation vector is simply

$$
\theta=\gamma B_{1} \Delta T
$$

where $\Delta T$ is the duration of the hard pulse.

In order to gain information on the nuclei in a sample, there are several parameters which can be manipulated. The magnitude of the magnetisation vector is governed by a set of equations called the Bloch equations, which can be used to calculate intrinsic properties of a nucleus. These equations, and the intrinsic relaxation times of the nuclei are discussed in the following sections.

\section{Separable solution of Bloch Equations}

The Bloch equations are a set of equations that describe the behaviour of the magnetisation vector of a nucleus in a magnetic field. As described above, the magnetisation vector has both a longitudinal and transverse component. The time evolution of these components following application of a radio frequency pulse is given below:

$$
\begin{gathered}
M_{x y}=M_{x y, 0} e^{-t / T_{2}} \\
M_{z}=M_{z, \mathrm{i}} e^{-t / T_{1}}+M_{z, 0}\left(1-e^{t / T_{1}}\right)
\end{gathered}
$$


where $M_{z, \mathrm{i}}$ is the initial longitudinal magnetisation following excitation, $M_{z, 0}$ is the equilibrium magnetisation in the longitudinal plane, $M_{x y, 0}$ is the initial transverse magnetisation following excitation, and $T_{1}$ and $T_{2}$ are the longitudinal and transverse relaxation times respectively.

\section{$T_{1}$ and $T_{2}$}

$T_{1}$ refers to the time taken for the longitudinal magnetisation of a sample to recover to $1-1 / e$ of its maximum value. That is, a sample with a $T_{1}$ of 1 second, such as water, will take 1 second to recover the longitudinal component by $63 \%$. Similarly for $T_{2}$, the transverse component of the sample will take one $T_{2}$ length of time to decay by $63 \%$.

\subsubsection{MRI Pulse Sequences}

The work in this project involved images obtained using several common MRI pulse sequences. These pulse sequences and acquisition techniques involved in reconstructing these images are outlined in this section, including a general description of inversion recovery and gradient echo images, and Siemens VIBE and TWIST images.

\section{GRE and SPGR}

Gradient echo (GRE) refers to a pulse sequence in which two gradients are applied in order to 'reverse' an FID through dephasing and rephasing.

Spoiled gradient echo refers to a gradient echo pulse sequence in which the transverse components of magnetisation are 'spoiled', or destroyed by an RF spoiling pulse, in order to remove the transverse component of the magnetisation before each RF pulse. The benefit of this is in producing $T_{1}$ weighted images through selection of $T_{R}, T_{E}$ and the flip angle.

The signal from a spoiled gradient echo pulse sequence is governed by the SPGR signal equation: 


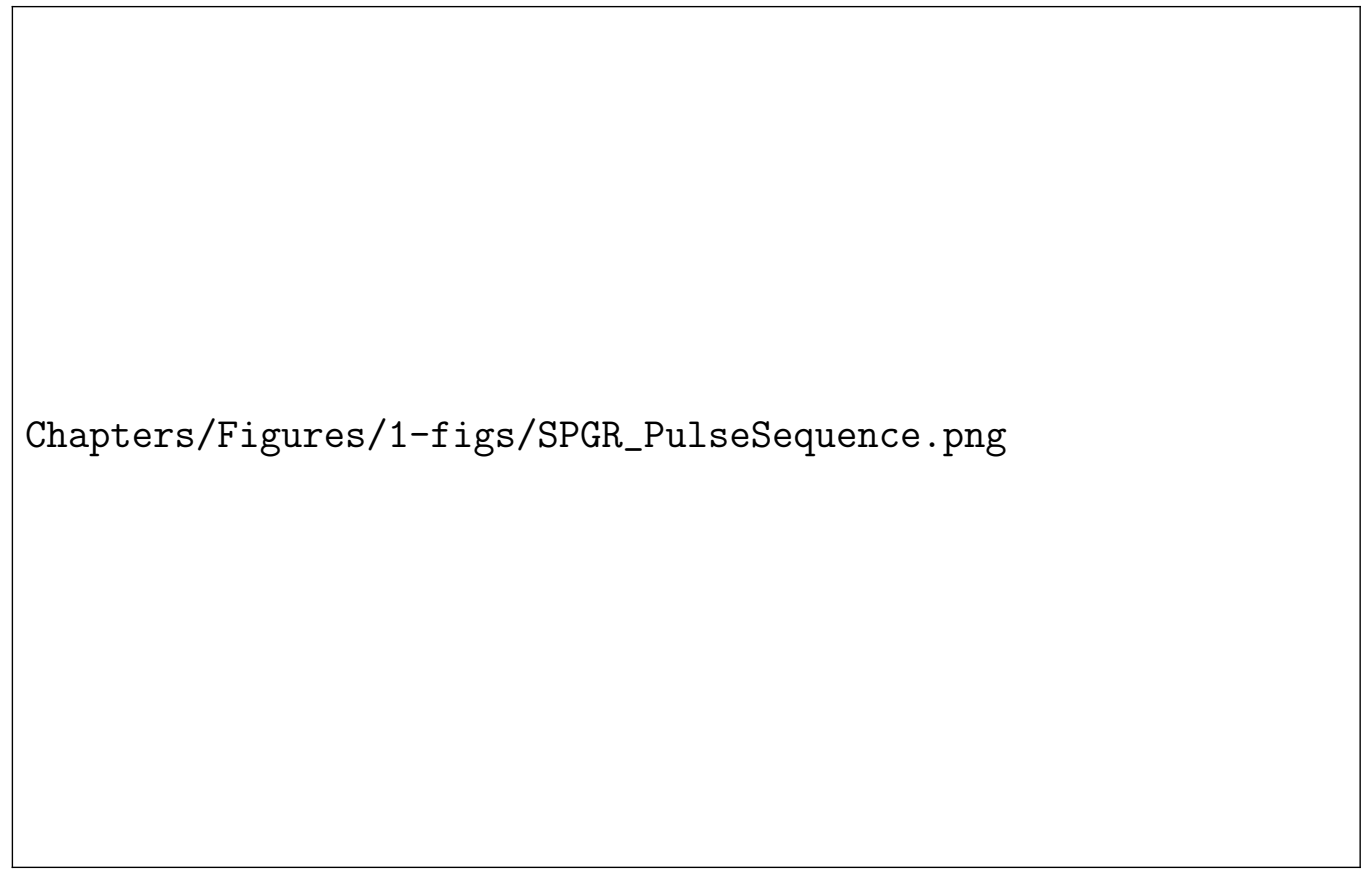

Figure 2.4: Pulse sequence diagram of spoiled gradient echo [31]

$$
S=S_{0} \frac{\sin \alpha\left(1-e^{\frac{-T_{R}}{T_{1}}}\right)}{1-(\cos \alpha) e^{\frac{-T_{R}}{T_{1}}}} e^{\frac{-T_{E}}{T_{2}^{*}}}
$$

where $\alpha$ is the flip angle of the DCE series. Through selection of sufficiently small $T_{E}$, the $e^{\frac{-T_{E}}{T_{2}^{*}}}$ term approaches unity and its effects can be neglected.

\section{Inversion Recovery}

The inversion recovery sequence in MRI is a pulse sequence used with varying inversion times, $T_{I}$ from which $T_{1}$ of a sample can be measured.

Through the use of different inversion times, the longitudinal magnetisation is allowed to relax by different amounts prior to inversion and measurement, and with knowledge of the inversion times at each acquisition, $T_{1}$ can be accurately determined with an inversion recovery sequence. 


\section{VIBE}

A Volumetric Interpolated Breath-hold Examination (VIBE) is the vendor name given to Siemens' fast gradient echo pulse sequence. This sequence is a $3 \mathrm{D}$ spoiled gradient echo sequence which produces $T_{1}$ weighted images. In this project, the VIBE sequence was used for acquisition of variable flip angle images from which $T_{1,0}$ maps were calculated.

\section{TWIST}

TWIST (Time-resolved angiography With Interleaved Stochastic Trajectories) is the vendor name for Siemens' high temporal resolution spoiled gradient echo pulse sequence, designed for fast acquisition of temporally resolved, contrast enhanced angiography. The underlying pulse sequence in TWIST is the spoiled gradient echo pulse sequence described previously, and the implementation of this sequence in this project is described in Section 3.2.

The k-space sampling method used in TWIST is a type of keyhole imaging that allows for fast imaging of angiography. This sequence has temporal resolution capabilities of sub one second, however, due to the trade-off between spatial and temporal resolution, temporal resolution in this project was considerably slower.

\subsection{DCE-MRI Acquisition and Qualitative Anal- ysis}

Perfusion can be mapped through the acquisition of spatio-temporal MRI data. Dynamic Contrast Enhanced (DCE) is one imaging method for perfusion MRI, which allows for the extraction of quantitative haemodynamic parameters associated with perfusion [32]. In this Section the acquisition of DCE-MRI is detailed, followed by an introduction to qualitative and semi-quantitative DCE-MRI analysis techniques. 


\subsubsection{DCE-MRI Acquisition}

DCE-MRI consists of a time series of $T_{1}$ weighted images obtained before, during, and after the injection of a Gadolinium based contrast agent. A power injector is typically used for the injection of contrast agent, with a standard contrast agent concentration for a subject being $0.1 \mathrm{mMol}$ per $\mathrm{kg}$ [33]. The specific acquisition parameters of the DCE-MRI series analysed in this project are provided in Section 3.2, whereas the present Section will cover the general requirements and constraints of DCE-MRI acquisition in order to obtain images that will allow for quantitative analysis.

\section{Relationship between signal and concentration}

The contrast agent typically used in DCE-MRI has a very short relaxivity, $R_{1}$, which affects the signal detected in the $T_{1}$ weighted images as the bolus of contrast passes through the vasculature over time. The relationship between signal enhancement in the DCE-MRI images and the concentration of contrast agent $(\mathrm{mMol} / \mathrm{L})$ can only be approximated to be linear at low concentrations [34], as the MRI signal data, $S(t)$, is a measure of the contrast agent's effect on the relaxation times rather than the concentration of contrast agent.

Depiction of the error that arises in measurements of magnetisation magnitude signal at higher contrast agent concentrations is given in Figure 2.5.

As such, although linear approximations exist in converting DCE-MRI signal to contrast agent concentration [34], in this project a non-linear conversion method was used, and is detailed further in Section 4.4.1. A second consequence of the non-linearity between signal and concentration is that at high concentrations, measurements of signal will eventually saturate, a factor of particular relevance in AIF estimates.

\section{Spatio-temporal Resolution Considerations}

The pulse sequences in DCE-MRI are generally a gradient echo sequence allowing for acquisition of $T_{1}$ weighted images [36]. As is the case in most 
Chapters/Figures/1-figs/PLACEHOLDER nonlinearRegime.png

Figure 2.5: Depiction of linear and non-linear regime between measured and actual concentrations of gadolinium based contrast agent [35] 
MRI acquisitions, a balance must be struck between spatial and temporal resolution.

Here, temporal resolution refers to the temporal spacing between subsequent images in a DCE-MRI time series. The temporal resolution of the DCE-MRI time series impacts which perfusion parameters may be extracted. The precise definitions of these perfusion parameters are provided in Section 2.4.1, however in general if we wish to measure flow of plasma, which is on the order of a few seconds, the temporal resolution of the images must be lower than this [37]. A reference point for the minimum temporal resolution required for measurement of plasma flow is 2 seconds or lower [38]. Conversely, slower physiological processes such as the uptake and washout of a bolus of contrast agent, which in this project was on the order of 25 seconds from bolus arrival time (BAT) to peak concentration, have lower demands on temporal resolution. However, for these slower processes we require that the total acquisition time is sufficient to capture the signal enhancement effects of the process of interest [37].

In terms of spatial resolution, we require sufficient resolution such that:

1. we are able to distinguish tissues of interest, and

2. there is a voxel entirely situated within an artery to serve as an AIF with negligible partial volume effects.

There are many techniques allowing for better compromises between spatial and temporal resolution, such as parallel imaging, zero padding, or limiting the field of view to only the slices of interest [37].

\subsubsection{Qualitative and semi-quantitative DCE-MRI anal- ysis}

As the contrast agent is carried in the blood plasma, cerebral perfusion can be measured through analysis of the uptake and washout of contrast agent within a tissue of interest. The curve produced by plotting a voxel's signal 


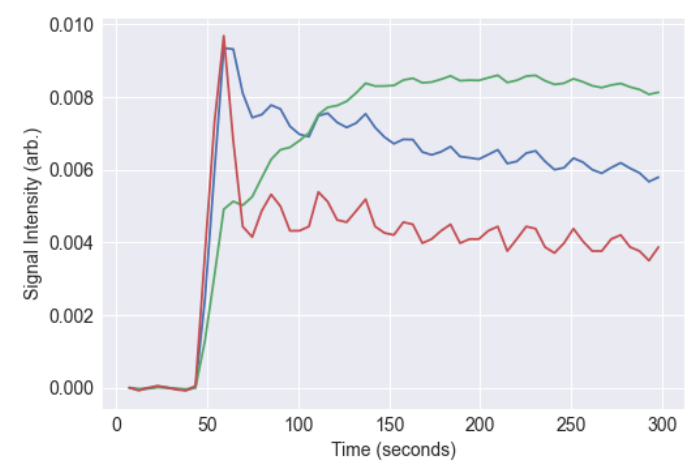

Figure 2.6: Examples of different shapes of signal enhancement curves.

intensity, $S(t)$, over time is called the signal enhancement curve and analysis of these curves can give us information on the type of tissues present within a voxel.

There exist several qualitative and semi-quantitative methods of analysing these signal enhancement curves to extract information on the underlying tissue. The goal of this project was to quantify perfusion to allow for interanimal comparison and quantitative characterisation of the animal model under consideration. As such, the qualitative and semi-quantitative methods presented in this Section were not applied in this project, but these methods allow for quick, model-free estimation of tissue characteristics and as such are presented here, with a full explanation of the quantitative analysis techniques detailed in Section 2.4.

\section{Qualitative Analysis Techniques}

Qualitative methods for analysis of signal enhancement curves typically involve classification of the curve into one of three types, as shown in Figure 2.7.

With prior empirical knowledge of the typical signal enhancement behaviour of different types of tissue, physiological information can be gained simply from classifying the curve shape. For example, in the classification of breast lesions, a type I curve indicates a benign lesion whereas a type III 


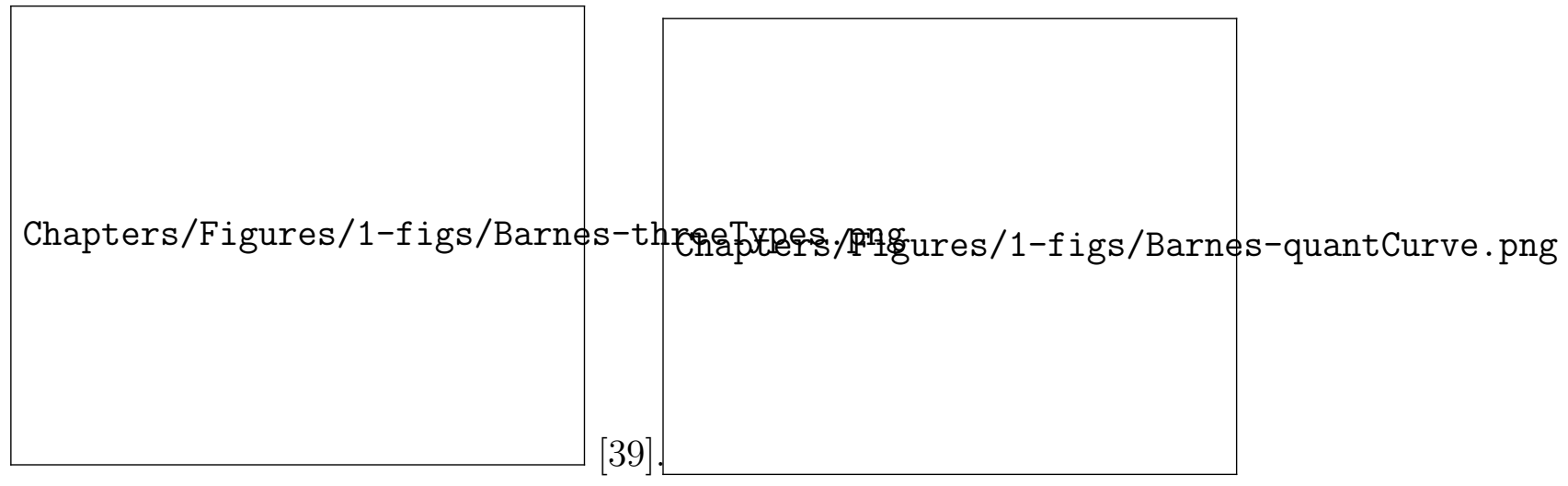

(a)

(b)

Figure 2.7: (a) Three types of curve classification, Type I- persistent, II- plateau and III- washout (b) semi-quantitative measurements from signal enhancement curve [40]

curve is a strong indicator for malignancy [41].

\section{Semi-quantitative Analysis Techniques}

Semi-quantitative analysis methods involve measurement of curve shape parameters such as time to peak, wash-in or wash-out slope, or area under the curve (AUC). Similarly to qualitative methods, comparison to known values for healthy or diseased tissue allows for fast classification or diagnosis in a clinical setting [42][41].

These methods allow for information on the tissue to be gained simply from the enhancement curve without further processing required, and typical healthy or diseased curve types and semi-quantitative curve measurements in various malignancies are well documented [43]. However, this type of analysis of the signal enhancement curve does not give any information on the underlying physiology of the tissue. For this, quantitative methods are required. 


\subsection{Quantitative DCE-MRI Analysis}

Quantifying perfusion in DCE-MRI is a much more involved process than the qualitative and semi-quantitative approaches detailed in Section 2.3.2, but allows for estimation of physiological parameters associated with perfusion through the fitting of pharmacokinetic model parameters. This process requires conversion from DCE-MRI signal to physiological units of contrast agent $(\mathrm{CA})$ concentration per $\mathrm{mL}$ of blood, described further in Chapter 4. Following this conversion, pharmacokinetic modelling of the tissue is undertaken in order to estimate quantitative perfusion parameters. The tracer kinetic theory behind the formation of pharmacokinetic models, some commonly used models, and the parameters we may estimate from application of these models are detailed in this Section.

\subsubsection{General Introduction to Tracer Kinetics}

Tracer kinetic theory is used to describe the passage of a tracer, in this case a bolus of contrast agent, as it passes through the tissue of interest. This Section will provide a brief introduction to the tracer kinetics, which will be built upon in the following Section on pharmacokinetic models of perfusion.

The Gadolinium based contrast agent used in this project has very small molecular size [44] allowing it to pass out of the intravascular space and into the interstitial space without entering the intracellular space. These different spaces are visually represented in figure 2.8 .

\section{Cerebral blood flow $-F_{\mathrm{p}}$}

Within the intravascular space, the contrast agent is carried in the plasma, the flow of which is described by the haemodynamic parameter $F_{\mathrm{p}}$ [44], referred to in this project as the cerebral blood flow. The fraction of blood that is plasma is defined by the haematocrit, and in humans this value is typically 0.45 [45]. $F_{\mathrm{p}}$ has units of $\mathrm{mL}$ of plasma flowing through $100 \mathrm{~mL}$ of tissue per minute, or $\min ^{-1}$. 


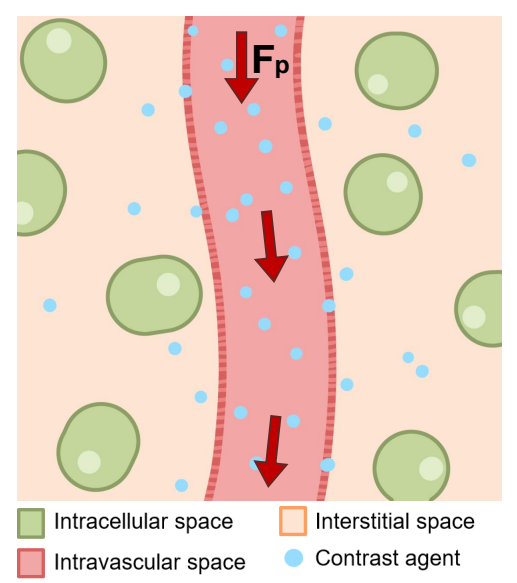

Figure 2.8: Simplified representation of the intravascular space, interstitial space and intracellular space and the passage of a tracer, carried in the plasma by flow $F_{\mathrm{p}}\left[\mathrm{min}^{-1}\right]$.

\section{Permeability surface area product - PS}

The transit of the contrast agent between the intravascular and interstitial space is described by the permeability surface area product, PS [44]. The rate at which tracer moves out of the intravascular space and into interstitial space, and the rate of backflow from the interstitial space to the intravascular space are assumed to be symmetric, and this assumption of symmetry is common to all current pharmacokinetic models [37]. This parameter has the same units as $F_{\mathrm{p}}$ of $\min ^{-1}[44]$.

\section{Fractional Volume Parameters}

In DCE-MRI, the parameters associated with the relative volumes of the interstitial and intrasvascular space, as depicted in Figure 2.8, are generally estimated as a fraction of total tissue volume [44]. That is, the parameter $v_{\mathrm{e}}$ describes the fraction of unit tissue volume that is made up of interstitial space, the subscript 'e' denoting extracellular-extravascular space or EES, another name for the interstitial space. Similarly, the fractional plasma volume, $v_{\mathrm{p}}$, describes the fraction of unit tissue volume that is intravascular 
plasma space (IVPS) [44].

\section{The Tissue Concentration Curve}

The concentration of contrast agent within the tissue (in mMol of contrast agent per $\mathrm{mL}$ of tissue) is referred to as a voxel's tissue concentration curve and is denoted $C_{\mathrm{t}}(t)[46]$. An alternative expression of the amount of contrast agent within tissue over time is given by the residue function.

\section{The Residue Function}

The residue function, $R(t)$ describes the fraction of the contrast agent bolus remaining within the tissue at time $t$ [46]. This function is therefore zero at any time before the bolus arrival time (BAT), and reaches $100 \%$ or 1 following the arrival of the bolus [44].

\section{The Impulse Response Function}

The impulse response function, $H(t)$, describes the intrinsic perfusion characteristics of the tissue, or in our case, the response of the tissue to the arrival of the contrast agent bolus. In tissue we assume a linear and stationary system, that is, that the response of the tissue to the tracer arrival is proportional to the input dose of tracer, and that the response of the tissue is time independent, or intrinsic to the tissue [44].

This impulse response function is the function we wish to fit in DCE-MRI analysis. However, as the response of the tissue varies with the input of contrast agent, in order to estimate the impulse response function, we must first obtain an estimate of the input function.

\section{The Arterial Input Function}

The input of the contrast agent bolus to the tissue of interest is described by the arterial input function (AIF). This function is crucial to quantification of DCE-MRI data, as without an estimate of it, we can not remove the 
dependence of our parameters on the shape of the bolus input [42]. That is, the measured tissue concentration curves, $C_{\mathrm{t}}(t)$, are masked by the input function of the bolus to the tissue.

Because of this, in order to calculate the impulse response function parameters that are intrinsic to the tissue and invariable with input function, we need an estimate of this input function. Estimation of the arterial input function (AIF) is a crucial step in DCE-MRI data analysis, as the AIF estimate is used in a deconvolution to obtain the desired impulse response function. There are several different methods for extracting the kinetic parameters of interest from DCE-MRI data, which are covered in detail in Chapter 5.

\subsubsection{Pharmacokinetic Models of Perfusion}

Quantitative DCE-MRI analysis is a model based, parametric technique which requires the use of a multi-compartmental model to represent the perfusion changes that take place at the capillary level. This Section builds upon the tracer kinetic theory concepts introduced in Section 2.4.1.

The parameters that may be estimated depend on the selection of pharmacokinetic model to represent the underlying tissue.

\section{General PK model introduction}

A pharmacokinetic model (PK model) of perfusion is a simplified version of the compartments and movement of fluid involved in perfusion at the capillary level. Different models make different assumptions about the volumes of certain compartments or magnitude of flows in or out of different compartments. Three pharmacokinetic models are presented in this section, each of which was considered for use in this project in Chapter 4.

\section{Two Compartment Exchange Model}

The two compartment exchange model is the most recently developed [42] and the most general [37] of the pharmacokinetic models considered in this 


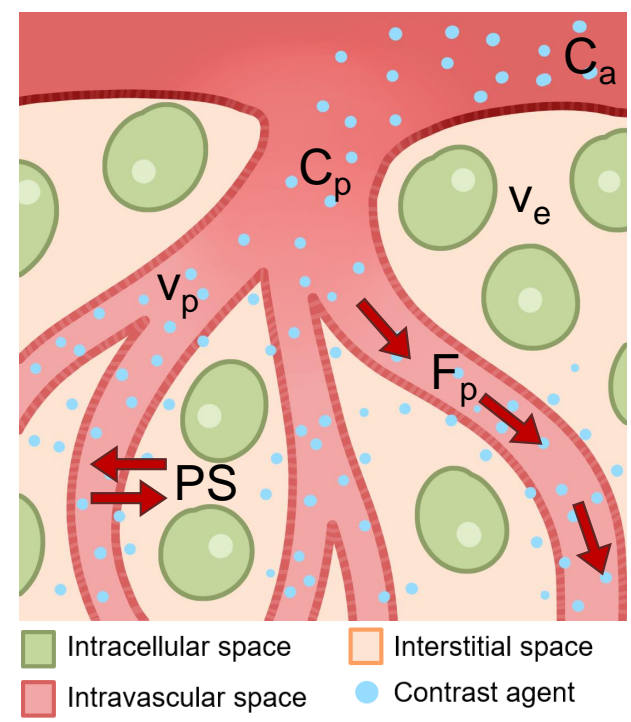

Figure 2.9: Diagram of two compartment model of perfusion with cerebral blood flow, $F_{\mathrm{p}}, \mathrm{PS}, v_{e}$ and $v_{\mathrm{p}}$.

project.

The relationship between the impulse response function and a tissue concentration curve in this model is given by

$$
C_{\mathrm{t}}(t)=F_{\mathrm{p}} H_{2 C X M}(t) * C_{a}(t)
$$

where $*$ denotes convolution, $C_{\mathrm{t}}(t)$ is the tissue concentration curve, $H_{2 C X M}(t)$ represents the impulse function specific to the two compartment model and $C_{\mathrm{a}}(t)$ is the AIF in a feeding artery [42]. It should be noted that in subsequent models, $C_{\mathrm{a}}(t)$ is denoted more generally as $C_{\mathrm{p}}(t)$, as it is assumed that plasma concentration in a feeding artery and in the intravascular plasma is indistinguishable [37].

The two compartment exchange model provides capability to separately estimate the parameters $F_{\mathrm{p}}$ and PS, however we require sufficient temporal resolution in our data to distinguish $C_{\mathrm{p}}(t)$ and $C_{\mathrm{a}}(t)$ for the model's assumptions to hold. The requirements on temporal resolution of the data to be fitted are discussed further in Section 4.5.2. 


\section{Tofts Model}

One of the original pharmacokinetic models of perfusion is the Tofts model [47]. This model simplifies the structures associated with perfusion to two compartments, the interstitial compartment and the plasma compartment, illustrated in Figure 2.10.

The interstitial compartment represents the extracellular extravascular space (EES) and the plasma compartment represents the volume of intravascular space that contains plasma, in which contrast agent is carried. In this model, it is assumed that the volume of this plasma compartment is negligible compared to the interstitial compartment volume. As such the parameters included in this model are $v_{e}$, the interstitial volume fraction, and the parameter $K_{\text {trans }}$. The impulse response function of tissue in this model is

$$
H_{\text {Tofts }}(t)=K_{\text {trans }} e^{\left(-K_{\text {trans }} t / v_{e}\right)}
$$

and the plasma concentration, $C_{\mathrm{p}}$ masks the tissue concentration curves in the form

$$
C_{\mathrm{t}}(t)=H_{\text {Tofts }}(t) * C_{\mathrm{p}}(t)
$$

where $*$ denotes convolution.

In the Tofts model the exchange of contrast agent between these two compartments is governed by the parameter $K_{\text {trans }}$, which is a combination of the effects of cerebral blood flow $\left(F_{\mathrm{p}}\right)$ and the vessel's permeability (PS). $K_{\text {trans }}$ therefore represents a combined estimate of these two parameters, and may reflect either depending on the physiological limitations of the situation. That is- in a permeability limited tissue, where changes in flow are negligible compared to changes in permeability, $K_{\text {trans }}$ reflects vessel barrier permeability. Alternately in flow limited tissue, where changes in vessel permeability are negligible compared to changes in flow, $K_{\text {trans }}$ will reflect changes in cerebral blood flow [48]. 


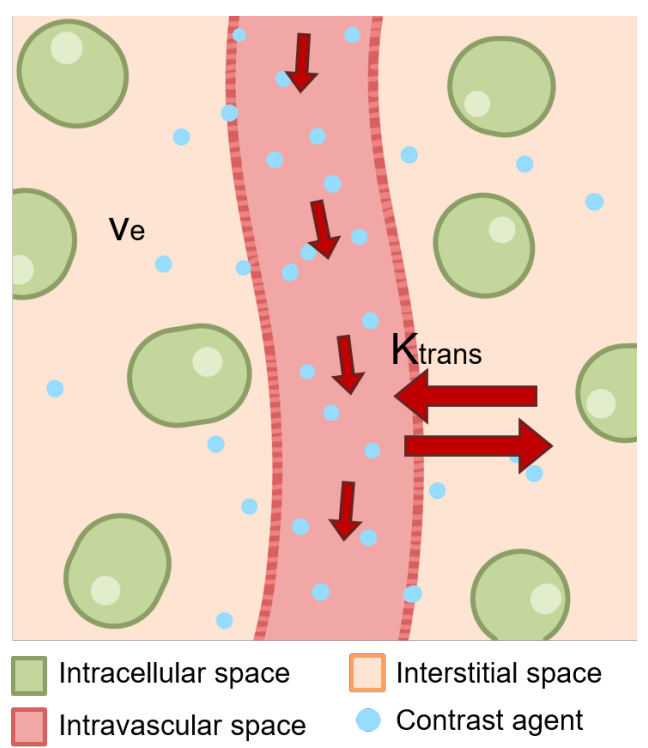

Figure 2.10: Diagram of original Tofts model of perfusion including parameters $K_{\text {trans }}$ and $v_{e}$.

\section{Extended Tofts Model}

An extension of the Tofts model to include the plasma volume fraction term, $v_{\mathrm{p}}$, is referred to as the extended Tofts model, which is one of the most commonly used models in DCE-MRI analysis [37][49]. This model, illustrated in Figure 2.11, allows for calculation of both the interstitial volume fraction and the plasma volume fraction, and perfusion of contrast agent between these compartments is governed by $K_{\text {trans }}$, as in the original Tofts model.

The form of the tissue impulse response function remains unchanged from Equation 2.7, but there is an additional $v_{\mathrm{p}} C_{\mathrm{p}}$ term included in the model of the tissue concentration curve:

$$
C_{\mathrm{t}}(t)=H_{\text {exTofts }}(t) * C_{\mathrm{p}}(t)+v_{\mathrm{p}} C_{\mathrm{p}}(t) .
$$

This $v_{\mathrm{p}} C_{\mathrm{p}}(t)$ term arises due to a key assumption of the extended Tofts model; that the cerebral blood flow is fast enough such that the transit time can be considered instantaneous [37]. Given this assumption, the fraction of 


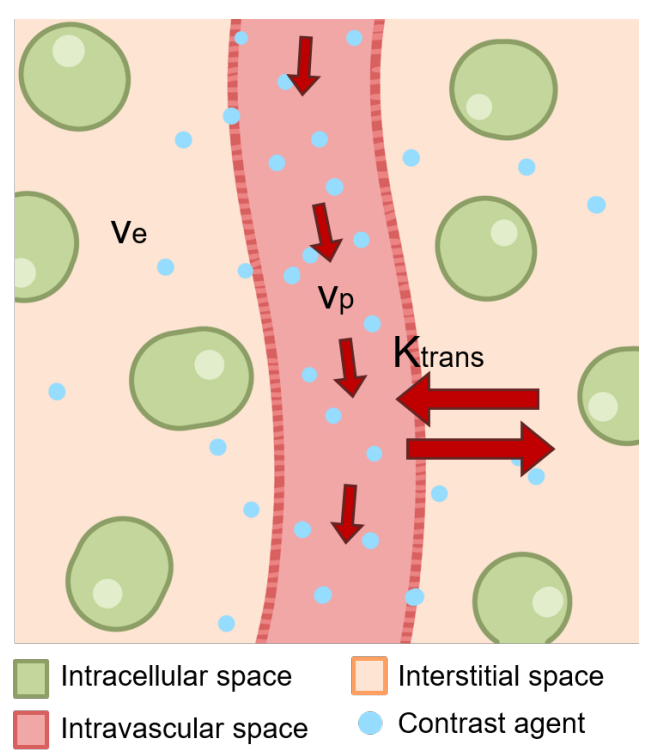

Figure 2.11: Diagram of three parameter extended Tofts model of perfusion including parameters $K_{\text {trans }}, v_{e}$ and $v_{\mathrm{p}}$.

contrast agent that does not pass out of the intravascular space is considered to be proportional to the contrast agent concentration in the plasma, due to the essentially infinite cerebral blood flow corresponding to instantaneous transit across the capillary bed [37].

\subsection{DCE-MRI in Stroke Literature Review}

DCE-MRI analysis techniques have been used in the context of stroke in past research. Estimates of expected perfusion parameter changes in stroke exist in literature, but the parameter estimates calculated as part of this project are the first calculated to characterise this novel ovine model of stroke. This Section presents a literature review of relevant research providing context on the use of these perfusion parameters.

A general baseline of the parameter $K_{\text {trans }}$ in the cerebral white/grey matter of healthy human subjects is $\approx 0 \mathrm{~min}^{-1}$ [50]. This is due to the 
presence of the specialised layer of endothelial cells in the brain forming the semi-permeable blood brain barrier (BBB) that carefully regulates the perfusion across this boundary. When intact, the BBB means that the permeability surface area product is low. For comparison, literature values for $K_{\text {trans }}$ in the healthy lung range from 0.05 to $0.1 \mathrm{~min}^{-1}$ [51], and in diseased lungs, this parameter may increase to $\leq 0.5 \mathrm{~min}^{-1}$ [52].

A rodent model of transient stroke found that the $K_{\text {trans }}$ values calculated from within the infarct at 24 hours post procedure were consistently higher than values in the contralateral hemisphere [53]. This was concluded to be due to the breakdown of the blood brain barrier following the transient middle cerebral artery occlusion.

Due to the combined nature of $K_{\text {trans }}$, many studies have used this parameter as a measure of blood brain barrier (BBB) permeability, such as Villringer et al in their study in human stroke patients. This research found the mean $K_{\text {trans }}$ values in the affected hemisphere, $0.7 \times 10^{-3} \mathrm{~min}^{-1}$, were more than three times as high as mirror $K_{\text {trans }}$ values, $0.2 \times 10^{-3} \mathrm{~min}^{-1}$ [4]. Another study similarly used $K_{\text {trans }}$ as a measurement of BBB permeability in determining if this parameter could predict hemorrhagic transformation following ischemic stroke [54].

Hemorrhagic transformation, or HT, refers to the restoration of cerebral blood flow to blood vessels damaged by ischemic stroke [55], and can be a serious complication following stroke. Hemorrhagic transformation provides an example of how interlinked different physiological processes are within the brain following stroke, as early BBB breakdown has been associated with both early HT and the severity of the HT [55].

Despite the common usage of $K_{\text {trans }}$ as a measure of BBB permeability, it has been noted that the use of $K_{\text {trans }}$ as a measurement of permeability in the context of stroke may produce inaccurate permeability measurements due to the fact that less contrast agent is available in the region of ischemic tissue [56]. As such, permeability measurements using $K_{\text {trans }}$ will reflect the reduced amount of available contrast agent rather than permeability of the vessel. 
The cerebral blood flow $\left(F_{\mathrm{p}}\right)$ takes into account this reduced amount of available contrast agent, as $F_{\mathrm{p}}$ describes the flow of plasma into the region of interest. As such, reduced $F_{\mathrm{p}}$ may affect the ability of $K_{\text {trans }}$ to be used as a measure of permeability.

Although cerebral blood flow in humans is carefully controlled by the process of autoregulation, the underlying mechanisms are known to be frequency dependent, with less buffering capacity against rapid or transient changes in blood pressure [57]. This is supported by studies in humans showing that cerebral blood flow recorded using high resolution transcranial doppler ultrasound exhibit high levels of shorter-term spontaneous fluctuations [58]. As such, it is possible that cerebral blood flow is contributing to measurements of $K_{\text {trans }}$ that were intended to reflect only barrier permeability. Physiological interpretation of $K_{\text {trans }}$ in this project is discussed further in Section 4.5, and estimation of $F_{\mathrm{p}}$ is highlighted as a relevant area of future research in characterising this animal model. 


\section{Chapter 3}

\section{Animal Experiments}

This project uses data that was obtained from animal experiments conducted at the University of Adelaide. This particular surgical model was an adaptation of a model of permanent stroke, which allowed for animal survival post-stroke, and involved a 2 hour transient occlusion. This process is detailed fully in [59] and is summarised briefly here, along with details of the MRI acquisition protocol.

\subsection{Surgical procedure}

This animal cohort consisted of 30 merino sheep (Ovis aries, 15F/15M, 18-36 months). Each animal was anaesthetised and a craniotomy performed at the junction of the parietal and squamous temporal bones. The proximal MCA was located and occluded for 2 hours with a mini aneurysm clip before being released to achieve reperfusion. The surgical site was closed following which animals were removed from anaesthesia and returned to indoor pens to recover. 


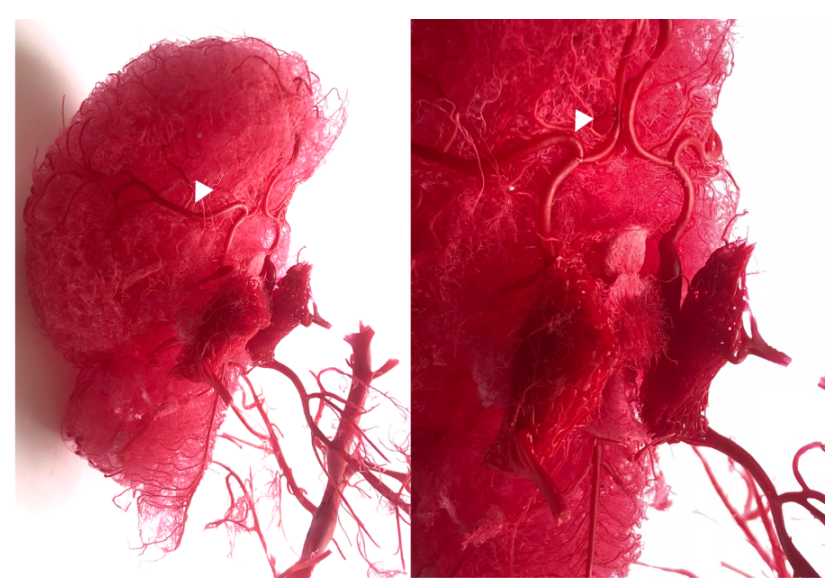

Figure 3.1: Resin cast of ovine cerebrovasculature. Middle cerebral artery is shown with the arrowhead in the left image, and the ideal location of surgical occlusion is denoted by the arrowhead in the right image.

\subsection{Image Acquisition}

21 of the 30 animals underwent MRI prior to surgery, and at 24 hours, 3 days, 6 days and 28 days post-stroke. At each acquisition animals were anaesthetised and scanning was performed on a 48 channel 3T Siemens Magnetom Skyra (Siemens Healthcare, Erlangen, Germany) with a posterior 20 channel head coil. The MRI protocol was a comprehensive, one and a half hour protocol which included acquisition of diffusion weighted images (DWI), $T_{1}$ weighted images, $T_{1}$ weighted DCE-MRI time series, $B_{0}$ field mapping and angiography images (MRA). DCE images were acquired with $T_{E} / T_{R}=1.98 / 5.06 \mathrm{~ms}$ and a flip angle of $12^{\circ}$. Injection commenced after acquisition of the second DCE scan, allowing for the acquisition of baseline signal prior to contrast enhancement. A gadolinium based contrast agent (Gadobutrol, commercial name Gadovist, Bayer, Australia) was administered via an intravenous catheter $(20 \mathrm{G}$, Terumo SURFLO) placed in the jugular vein. A power injector was used to administer gadolinium as a bolus (0.1 $\mathrm{mL} / \mathrm{kg} ; 3 \mathrm{~mL} /$ second) followed by a saline flush $(0.5 \mathrm{~mL} / \mathrm{kg} ; 5 \mathrm{~mL} / \mathrm{second})$. 
1 of the 21 animals that underwent imaging was euthanised prior to sufficient MRI data being obtained, leaving the cohort of 20 animals analysed as part of the present research. 


\section{Chapter 4}

\section{DCE-MRI Image Processing Methods}

The perfusion information of interest was contained within the DCE time series images but required several processing steps and inputs from other images before perfusion parameters could be calculated.

Some of these steps were:

- estimation of $B_{1}$ field inhomogeneity,

- calculation of $T_{1,0}$ maps,

- conversion from DCE signal image to $R_{1}$,

- conversion from $R_{1}$ to contrast agent concentration,

- determination of AIF estimate,

- conversion from AIF to $C_{p}$,

- pharmacokinetic model selection, and

- development of curve fitting techniques to the chosen model. 
Due to the different steps involved within AIF selection, these methods are covered separately in Chapter 5 . The specific methods pertaining to the remaining processing steps listed above are covered in this Chapter, along with the image processing techniques and tools used to process the DCE images.

\subsection{Overview of Image Processing Tools}

There are many existing DCE-MRI analysis tools with functionality to perform several of the image processing tasks presented in this chapter. These programs served as a useful validation technique, particularly for synthetic data results, but due to the number of images and different formats required for each program, ultimately the majority of image processing was conducted using Python written as part of this project.

This Section will briefly outline the different software packages and programming languages used in this project.

\section{Python 3}

The main processing tool used was a series of Jupyter notebooks written in Python 3. The notebook format allowed for quick tests of various methods, batch processing of several animals, and access to various existing Python libraries to process the different image formats used throughout the project. These formats included NIfTI, tif and DICOM stacks.

The Python code written in Jupyter was also converted into a multiprocessing capable Python file which was able to be run using Victoria University of Wellington's high performance computing cluster. This allowed for much more powerful image processing and was used to process all $90+$ $4 \mathrm{D}$ images at the final stages of analysis to output the extended Tofts model parameters. 


\section{ROCKETSHIP}

After evaluating functionality of several software packages, the Matlab plugin ROCKETSHIP ([60]) was the most appealing for use in this project due to the batch processing capability and user friendly GUI. ROCKETSHIP has functions for $T_{1}$ map generation from multi flip angle images, as well as extended Tofts parameter fitting capability, with user defined or automatic AIF selection.

Parameter bounds and fitting parameters can be set in a user adjustable preferences text file, and there are many options for setting an AIF selection method, noise handling, and masking regions of interest.

A downside to the use of ROCKETSHIP in this project was the amount of time taken to fully process a dataset from DCE image to parameter maps. Even using Matlab's parallel pool toolbox, processing times were still unfeasible for the amount of images, and the fact that each image also required a user to set the injection duration in run A meant this process could not be fully automated. The final run in ROCKETSHIP, run D, allowed for batch processing of images, but required a .mat file as input which would be generated as part of earlier runs. It was possible to generate .mat files from the Python results, but this proved more time consuming than keeping all steps in one language and standard format throughout the processing.

Instead, methods from ROCKETSHIP were adapted from Matlab and used in the multiprocessing capable Python file which was run on the high performance computing cluster. ROCKETSHIP was used within Matlab in this project for calculation of three flip angle maps used for validation of $T_{1}$ maps in Section 4.3.2, and conversion from signal to concentration of the synthetic data described in Section 4.6.5.

\section{dcemri.jl}

dcemri.jl ([61]) is an opensource toolkit for DCE-MRI analysis that also has functionality for many of the processing steps described later in this chapter. 
This toolkit was used for validation of synthetic data results, but was not used for processing of any animal images in this project.

\section{ImageJ}

Image J [62] is an image processing program with many capabilities including ROI drawing and analysis, histogram analysis, and conversion to different image types. The Fiji distribution of ImageJ [63] was used at every stage of this project to display, analyse, save and modify data in many different formats. Particularly useful was the hyperstack plugin which allowed for easy processing of the four dimensional datasets, and the nifti1 plugin which allowed for conversion from DICOM to analyse/NIfTI format.

\section{D Slicer}

3D Slicer [64] is predominantly a 3D image visualisation tool which also allows for segmentation, binary mask generation and image registration. 3D Slicer was used in this project for many processing steps, such as coregistering MRA image series with DCE image series, exporting binary masks and visualising structures in 3D.

\section{2 $\quad B_{1}$ Field Inhomogeneity Consideration}

The method for $T_{1}$ map calculation detailed in Section 4.3 relies on the assumption that the flip angle experienced at each point in the image is identical. This assumption requires that the $B_{1}$ field applied for excitation is perfectly homogeneous throughout the imaging volume. In order to verify that this assumption is valid, we require data on $B_{1}$ maps, either in order to confirm that this field was sufficiently homogeneous such that any impact on the $T_{1}$ map would be negligible, or in order to correct for any inhomogeneities seen in the $B_{1}$ maps.

The animals for which perfusion maps were generated as part of this 
project did not have $B_{1}$ field maps obtained as part of the MRI acquisition protocol. Due to the fact that data on the $B_{1}$ field was not available, a decision had to be made between making one of two assumptions.

1. Assume the $B_{1}$ field was effectively homogeneous within the region of interest, and the effect of any inhomogeneities on $T_{1}$ values will be negligible.

2. Assume any inhomogeneities in the $B_{1}$ field during acquisition of the first cohort of animals will be comparable to the $B_{1}$ field inhomogeneities seen in a second cohort of animals for which $B_{1}$ field maps were obtained.

The second assumption listed here was taken to be preferable, on the basis that the second cohort of animals was imaged at the same facility and had identical MRI acquisition procedures for the variable flip angle images as the cohort under investigation in this project. Investigation into $B_{1}$ field inhomogeneities in the second animal cohort was conducted, with the intention that flip angle discrepancies arising from $B_{1}$ inhomogeneity in these images would allow for estimation of uncertainties in the first cohort.

\subsection{1 $\quad B_{1}$ field inhomogeneities in a second animal co- hort}

The animal cohort under investigation in this Section, hereafter referred to as the second animal cohort, is different from the animals for which perfusion parameters are being calculated in this project, the primary cohort.

The second animal cohort were imaged at the same facility as the primary cohort (SAHMRI, Adelaide), but were imaged only once following surgery. The same MRI protocols for acquisition of variable flip angle images (T1_VIBE) was used as the primary cohort, allowing for direct comparison of these values, however these animals also had $B_{1}$ maps included in the protocol, as well as a third variable flip angle image. This extra data was used in this project for validation of methods on the primary cohort of sheep, particularly for the 
comparison of two to three flip angle $T_{1}$ mapping discussed further in Section 4.3 .2 .

For each animal in the second cohort, a $B_{1}$ map was obtained with $T_{E} / T_{R}=1.83 / 5000 \mathrm{~ms}$ and a flip angle of $8^{\circ}$. The change in flip angle with $B_{1}$ is described by

$$
\theta(x, y, z)=\theta_{\text {nominal }} \times B_{1 \text { rel }}(x, y, z)
$$

where $\theta(x, y, z)$ is the flip angle experienced at voxel $(x, y, z), \theta_{\text {nominal }}$ is the expected flip angle given a homogeneous field, and $B_{1 \text { rel }}$ is the value of relative change in $B_{1}$ as measured from the normalised $B_{1}$ map.

Applying this equation to the 2 degree and 15 degree variable flip angle images, it was found that with the relative $B_{1}$ inhomogeneities measured from within the brain in the second cohort of sheep, we would see a change in flip angle of $\pm 8 \%$. This finding gave support to the method of neglecting any $B_{1}$ inhomogeneities in the primary cohort of sheep as being small enough to not significantly influence $T_{1}$ maps.

\subsection{T1 Map Generation}

The first step of DCE-MRI analysis was the generation of a $T_{1,0}$ map for each animal at each time point, which is required as input for the conversion of the DCE-MRI images from signal intensity to concentration.

\subsubsection{Algebraic $T_{1}$ determination}

The images used for calculating $T_{1,0}$ maps were dual flip angle T1 weighted images $\left(T_{E} / T_{R}=4.09 / 1.47 \mathrm{~ms}\right)$ obtained immediately prior to DCE-MRI acquisition. The signal intensity of these images is governed by the spoiled gradient echo signal equation introduced in Section 2.2.2 and reproduced here, 


$$
S=S_{0} \frac{\sin \alpha\left(1-e^{\frac{-T_{R}}{T_{1}}}\right)}{1-(\cos \alpha) e^{\frac{-T_{R}}{T_{1}}}} e^{\frac{-T_{E}}{T_{2}^{*}}}
$$

The chosen echo time was short enough that the $e^{\frac{-T_{E}}{T_{2}^{2}}}$ was sufficiently close to 1 and could effectively be removed from the equation, giving

$$
S=S_{0} \frac{\sin \alpha\left(1-e^{\frac{-T_{R}}{T_{1}}}\right)}{1-(\cos \alpha) e^{\frac{-T_{R}}{T_{1}}}} .
$$

At each MRI acquisition, two such VIBE images were acquired at flip angles $\alpha_{1}=2^{\circ}$ and $\alpha_{2}=15^{\circ}$ with the identical number of slices and slice locations as the DCE-MRI series that followed. As the dual flip angle T1_VIBE images had the same echo and repetition times, the only unknown in this equation aside from $T_{1}$ was the $S_{0}$ term. $S_{0}$ refers to the equilibrium signal obtained using full excitation and signal recovery. As this term depends only on proton density and machine configuration settings which remained constant between acquisitions, $S_{0}$ was assumed to be the same for both flip angle images.

Rearranging of Equation 4.3 written for two flip angle values, $\alpha_{1}$ and $\alpha_{2}$, setting $S_{0,1}$ and $S_{0,2}$ as equal, and cancelling the exponential term in each denominator gave Equation 4.4,

$$
T_{1}=T_{R} \ln \left(\frac{S_{2} \sin \alpha_{1} \cos \alpha_{2}-S_{1} \cos \alpha_{1} \sin \alpha_{2}}{S_{2} \sin \alpha_{1}-S_{1} \sin \alpha_{2}}\right)^{-1},
$$

which allowed for voxel by voxel calculation of $T_{1}$.

\subsubsection{Verification of $T_{1}$ map values}

The range of flip angles used for $T_{1}$ map generation ranges from 2-7 in previous research [65], but the use of 3 or more flip angles allows for a least fit squares method rather than an algebraic method as used in this project. As such, it was desirable to confirm that the method using two flip angles was comparable 
to other methods such as inversion recovery or methods using more than two flip angles. Ideally an inversion recovery sequence would have allowed for the most accurate $T_{1}$ map generation, however due to machine time restrictionsthis sequence was not able to be obtained for all animals, and instead two flip angle images were obtained for each animal.

\section{Gadovist Sample Verification of $T_{1,0}$ Methods}

In order to verify that the $T_{1}$ values generated using the dual flip angle algebraic method given in Section 4.3 were comparable to those found using inversion recovery, dual flip angle $T_{1}$ values were calculated in a simple phantom and compared to the $T_{1}$ values using an inversion recovery sequence on an in-house 9.4T Bruker magnet system (Bruker, Germany). This sequence used 10 different inversion times ranging from 0.01 to 15 seconds. The phantom consisted of four test tubes of Gadovist at four different concentrations (0.05, 0.10, 0.15 and $0.20 \mathrm{mMol} / \mathrm{L})$. A dual flip angle $T_{1,0}$ map was generated with flip angles 2 and 10 degrees and compared to values calculated from the inversion recovery sequence.

From Figure 4.1, we see the $T_{1}$ values calculated using dual flip angle images approximated the values calculated using inversion recovery well. This result gave us confidence to move forward with the use of the dual flip angle algebraic $T_{1,0}$ map method.

\section{Comparison of Dual flip angle to Triple Flip Angle $T_{1,0}$ Maps}

In order to verify that the algebraic $T_{1,0}$ generation method using just two flip angles was equivalent to a fitting method with more flip angle images, three variable flip angle images were obtained in a second cohort of animals and $T_{1}$ maps were generated for these animals using both two and three flip angle images.

Comparison of the algebraic method using two flip angles, and the least squares fitting method using three flip angles was undertaken to validate the 


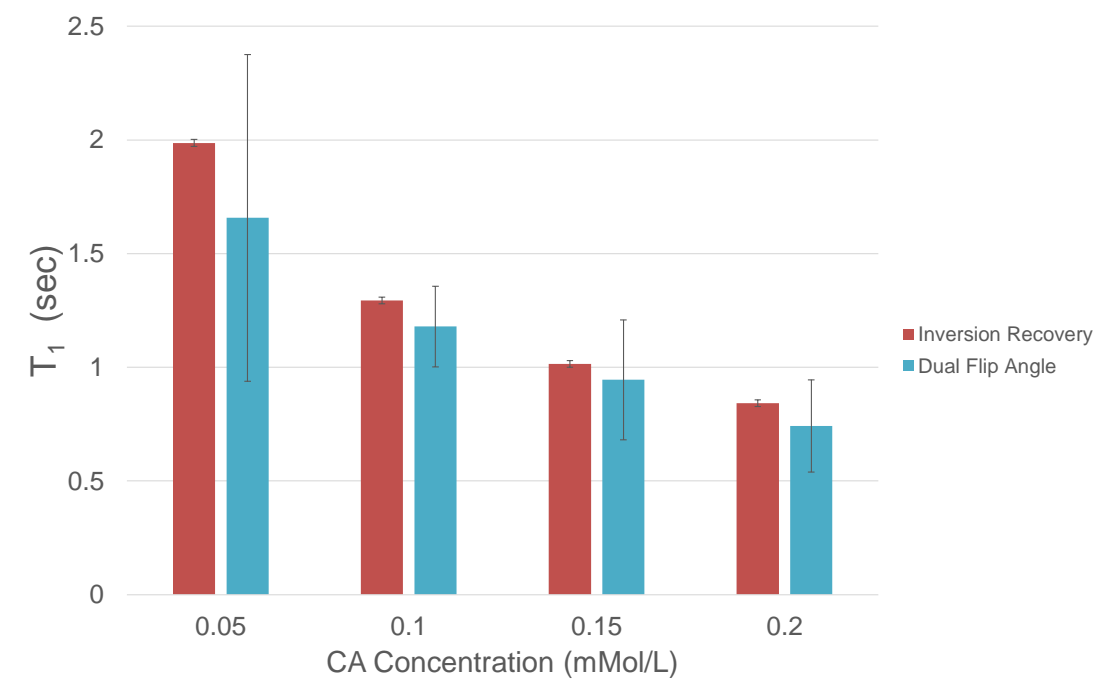

Figure 4.1: T1 values calculated from dual flip angle T1 map $( \pm$ standard error) and from inversion recovery sequence $( \pm$ standard deviation)

algebraic method, and investigate the uncertainty introduced from using two rather than three flip angle images.

As shown in Figure 4.2, the values calculated using the algebraic method used in this project, and the values using three flip angles match well within the region of interest; the cerebrum. This result gave us confidence to move forward in the analysis using the dual flip angle $T_{1,0}$ maps.

\subsection{Signal to Concentration Conversion}

The conversion from arbitrary signal intensity to contrast agent concentration with physical units of $\mathrm{mM} / \mathrm{L}$ involved several steps which are outlined here. 


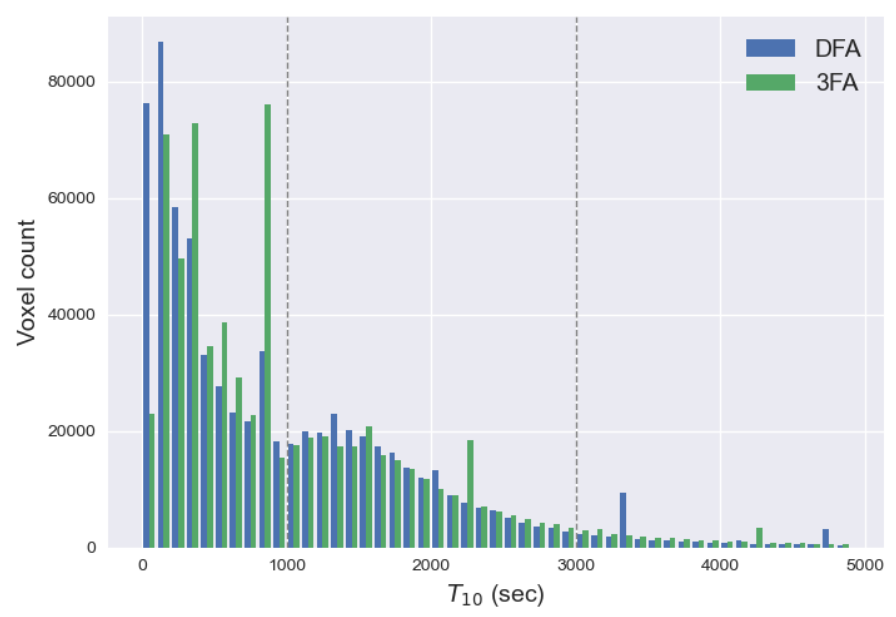

Figure 4.2: Histogram of dual flip angle $T_{1,0}$ map and three flip angle $T_{1,0}$ map generated for the same animal (MB004). The range of values that represent tissue within the animal's cerebrum is shown between the dashed lines.

\subsubsection{DCE signal to concentration conversion}

The DCE images acquired were $T_{1}$ weighted spoiled gradient recalled images (DCE_TWIST), with $T_{E} / T_{R}=5.06 / 1.98 \mathrm{~ms}$ and a flip angle of $12^{\circ}$. Similar to the T1_VIBE images introduced in the previous section, the signal intensity is governed by the spoiled gradient signal equation, but as the DCE images were a time series covering the duration of a contrast agent injection, the signal intensity of a voxel was a function of time, denoted $S(t)$.

Equations to convert DCE signal to contrast agent concentration were taken from the Quantitative Imaging Biomarkers Alliance DCE MRI Quantification Profile Document [65] and are given below:

$$
\begin{gathered}
\frac{1}{T_{1}(t)}=\frac{-1}{T_{R}} \times \ln \left(\frac{1-A}{1-\cos \theta A}\right) \\
A=B \frac{S(t)}{S(0)}
\end{gathered}
$$


where $S(0)$ is the baseline signal before contrast agent injection, and

$$
\begin{gathered}
B=\frac{1-E_{10}}{1-\cos \theta E_{10}}, \\
E_{10}=e^{\frac{-T_{R}}{T_{10}}} .
\end{gathered}
$$

The equation for conversion from $R_{1}(t)$ to concentration, $C(t)$, is given by:

$$
C(t)=\left(\frac{1}{T_{1}(t)}-\frac{1}{T_{1,0}}\right) \frac{1}{R_{G d}},
$$

where $1 / T_{1}(t)$ is the $R_{1}(t)$ as converted from the DCE series, using Equation $4.6,1 / T_{0}$ is the values given by the $T_{1,0}$ map calculated in Section 4.3, and $R_{\mathrm{Gd}}$ is the relaxivity of the Gadolinium based contrast agent used in the experiment.

\subsubsection{Determination of $R_{\mathrm{Gd}}$}

The contrast agent used in the animal experiments analysed in this project was Gd-DO3A-butrol (commercial name Gadovist, Bayer, Australia). Literature values for this contrast agent have been published at $3 \mathrm{~T}$, and range slightly from $4.2 \pm 3$ in water [66] to $5.0 \pm 0.3$ in plasma [67].

Different literature values are shown in Figure 4.3. The value selected for use in this project is also shown in this Figure, and was chosen to be 4.7 $\mathrm{mMol} / \mathrm{L}$. This value was within error for the Szomolanyi et al. experiments [69] shown as data point 4 in Figure 4.3, which best approximated physiological conditions (temperature, mixing of solution), albeit in human plasma rather than ovine plasma.

With the value of $R_{\mathrm{Gd}}$ to be used in this project determined, $R_{1}$ images could be converted to concentration using Equation 4.9. Following this conversion to concentration, the TCCs for each voxel were ready to be fit to the chosen pharmacokinetic model of perfusion. 


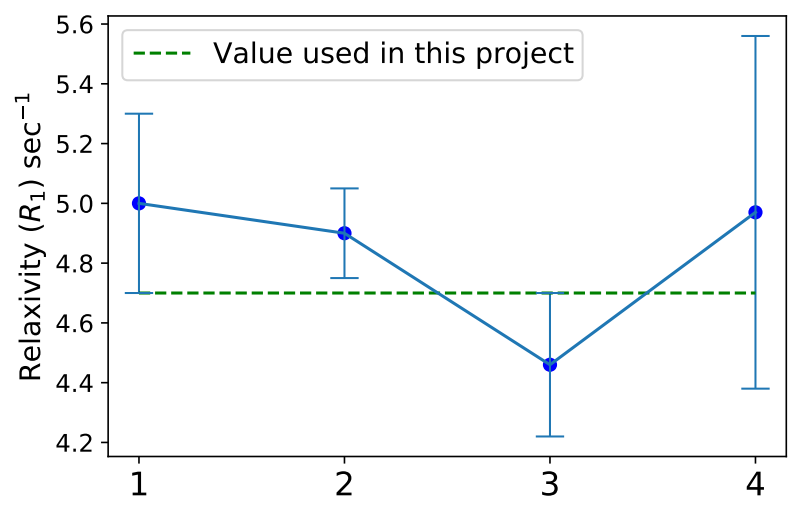

Figure 4.3: Different literature values for $R_{\mathrm{Gd}}$ at $3 \mathrm{~T}$ (from the left) 1 . Rohrer et al. [67] 2.Noebauer-Huhmann et al. [68] 3.Shen et al. [66] and 4. Szomolanyi et al. [69]

\subsection{Pharmacokinetic Model Selection}

There are many pharmacokinetic models of perfusion, some of which are presented in depth in Section 2.4.2, and each making different assumptions about the characteristics of the underlying tissue [37]. While some models allow for calculation of more parameters than others, these models also have higher requirements of the data in terms of signal to noise ratio and temporal resolution.

In this section, statistical and physiological considerations in pharmacokinetic model selection are presented. Statistical methods for pharmacokinetic (PK) model selection involved calculation of the Akaike information criterion (AIC) and Bayesian Information Criterion (BIC) for three potential pharmacokinetic models. Physiological considerations involved a literature review of appropriate acquisition thresholds for accurate estimation of perfusion parameters, and consideration of the physiological changes within the sheep's brain. The literature review proved to be crucial in determining whether a PK model was a valid choice for fitting in this project. This is because of 
the fact that a complex model with more parameters than a simpler model with fewer parameters will fit a concentration curve better, but does not necessarily provide reliable parameter estimates [37].

\subsubsection{Statistical Considerations in PK model selection}

In this section, the pharmacokinetic models under consideration were the Tofts model [70], the extended Tofts model [47] and the two compartment exchange model [36]. These models represent a two parameter, three parameter and four parameter model respectively. Other pharmacokinetic models considered in this project are discussed in Section 4.5.3.

In order to measure which out of these potential models was best, two estimators of error in fit were used. These were the Akaike Information Criterion (AIC) and Bayesian Information Criterion (BIC), the results of which are presented in this Section.

\section{Akaike Information Criterion}

The Akaike Information Criterion (AIC) is a statistical measure for reflecting which out of a set of potential models is the best choice for fitting. This criterion takes into account the number of independent variables, as well as the value of the residual of fit for each model. It provides relative preference for a set of models, but does not allow for calculation of absolute goodness of fit. As such, the model that returns the lowest AIC index can only be considered the best model out of the models tested, not necessarily out of all possible models.

In terms of sum of squared errors, the AIC is written as:

$$
\mathrm{A}=2 k+n \ln (\mathrm{R})
$$

where $k$ is the number of independent parameters in the model, $n$ is the number of samples and $\mathrm{R}$ is the sum of the residuals for each voxel, given by 


$$
\mathrm{R}=\sum_{t=1}^{n}\left(C_{\mathrm{t}}(t)-f(t)\right)^{2}
$$

where $\mathrm{C}_{\mathrm{t}}(t)$ is the tissue concentration curve at a given voxel, $f(t)$ is the fit produced by a given pharmacokinetic model to the tissue concentration curve and $n$ is the number of time points, which in this case was 57 for each voxel.

Taking a sample of 3764 voxels from the affected hemisphere, each with 57 time points, it was found that the Tofts model produced the lowest AIC for $31 \%$ of the voxels, the extended Tofts model produced the lowest AIC for $58 \%$ of the voxels and the two compartment model minimised the AIC for $1 \%$ of the voxels.

\section{Bayesian Information Criterion}

The Bayesian Information Criterion (BIC) is given by

$$
B=n \ln (R / n)+k \ln (n)
$$

where $B$ is the value for the BIC, $R$ is the sum of residuals as given by Equation 4.11, $k$ is the number of model parameters and $n$ is the number of samples, in this case, 57 time samples per tissue concentration curve. The BIC provides a harsher penalty for extra parameters than the AIC, and it was likely due to this reason that the BIC was minimised by the Tofts model for all voxels. The second best model for $100 \%$ of voxels sampled was the extended Tofts model, followed by the two compartment exchange model.

\section{Discussion of Statistical Approach to model selection}

Despite the fact that the 2 compartment model was able to consistently minimise the absolute sum of squared errors, the superiority of fit this model provided was overcome by the penalty applied for the model having four parameters in both the AIC and BIC. This penalty applied arises from the 
fact that if a model has more free parameters it is more likely to provide a good fit to data. This project aimed to extract meaningful physiological parameters from the data, and as such, selection of a model that simply provided a good fit without also allowing for meaningful interpretation of the fitted parameters was not appropriate.

The penalties applied for more parameters by these statistical criteria were valid for this reason, however in order to determine if certain model parameters were reasonable to attempt to extract, the physiological situation and the quality of the data had to be evaluated.

\subsubsection{Temporal Resolution in PK model selection}

The temporal quality of the data under analysis in this project was identified as a potential factor influencing which parameters could reasonably be extracted. The temporal resolution of the DCE-MRI series in this project was one acquisition of the volume of interest every 5.2 seconds.

In general, in order to estimate the parameter of cerebral blood flow, $F_{\mathrm{p}}-$ a parameter included in the two compartment exchange model, a temporal resolution of 2 seconds or less is recommended [37]. The minimum temporal resolution has been estimated to be even lower for methods involving deconvolution, which is all three pharmacokinetic models under consideration here, for which a temporal resolution of any greater than 1.5 seconds per image is insufficient [71].

The need for a minimum temporal resolution for estimation of flow is related to the transit time of a tracer, i.e. the time the tracer takes to cross from the arterial to the venous side of the capillary bed. If this parameter is on the order of 3-5 seconds, the recommended sampling interval for perfusion measurements is less than two seconds [38]. A study measuring transit times in humans found that this parameter in the brain ranged from 3.9-4.3 seconds using positron emission tomography, (PET), and 2.8-3.0 seconds when measured with DSC-MRI [72]. In terms of ovine transit times, one recent study has shown that this parameter in lambs and ewes is faster 


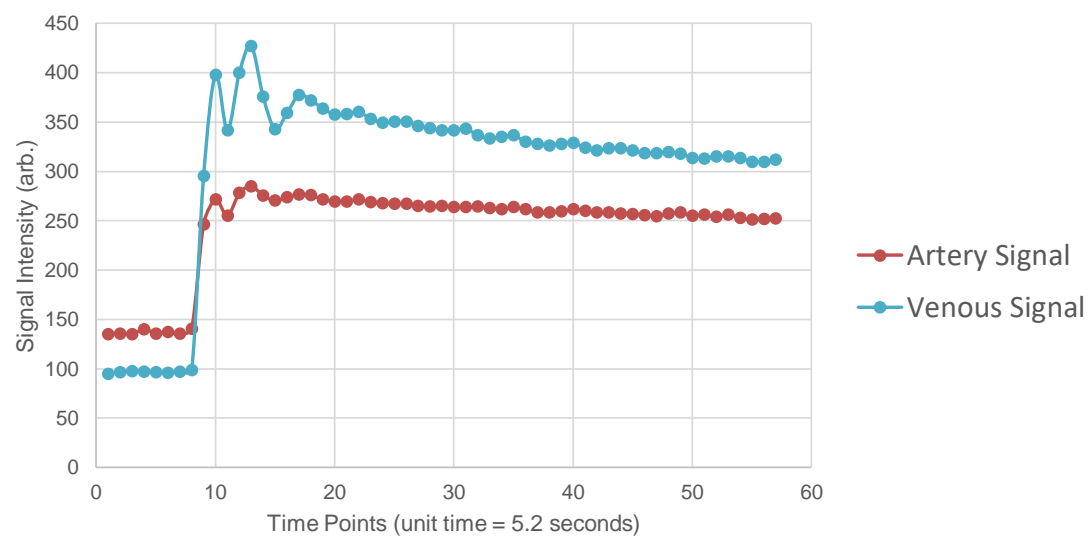

Figure 4.4: Plot of two signal enhancement curves, artery signal obtained from drawing a ROI from within the rete mirabile- upstream of the middle cerebral artery, and venous signal from the sagittal sinus.

than values previously reported in humans, which would demand an even faster temporal resolution for estimation [73]. Therefore, although the two compartment exchange model has the capability to provide estimates of $F_{\mathrm{p}}$ and PS separately, the data under analysis in this project would likely not allow for meaningful estimation of these parameters at its temporal resolution of 5.2 seconds.

This concept can be illustrated through analysis of the bolus arrival time (BAT) of tissue concentration curves obtained from the rete mirabile, located in the arterial tree upstream of the middle cerebral artery, and the large sagittal sinus visible in the posterior of the brain. With a temporal resolution sufficient to measure flow, we would expect a difference in BAT between arterial input and venous output curves, however in these images the BAT is indistinguishable, as shown in Figure 4.4, indicating that information on flow is being lost due to insufficient temporal resolution.

At this temporal resolution of 5.2 seconds, an assumption can be made that the transit time from arterial side to venous side of a capillary bed is effectively instantaneous. This results in a cerebral blood flow, $F_{\mathrm{p}}$, that 
is effectively infinite. It is this exact assumption that the extended Tofts model is based on, and that defines the parameter $K_{\text {trans }}$ [37]. The parameter $K_{\text {trans }}$ is described in more detail in Section 2.4.2, but is most importantly a measurement which provides a joint estimate of permeability and flow effects [42]. With the extended Tofts model's assumption of infinite cerebral blood flow, $K_{\text {trans }}$ reflects permeability changes [37], which, in the context of stroke, are known to occur in humans due to breakdown of the blood brain barrier following ischemic event.

\subsubsection{Discussion of PK model selection}

Between the Tofts and extended Tofts model, although the BIC indicated the Tofts model was preferable to the extended Tofts model, the underlying assumption that $v_{\mathrm{p}}=0$ in the Tofts model is known to be physiologically incorrect in post-stroke, particularly in the 6 days post-stroke and after. As such, taking into account both physiological and statistical considerations, the extended Tofts model was selected as the best model choice out of these three models.

Other pharmacokinetic models considered for use in this project include the Larsson model [74] and the Patlak plot model [75].

The Larsson model requires a gold standard AIF [42] which was not obtained for the animals in this project.

The Patlak plot model is a graphical model which assumes low permeability, which would not hold true following blood brain barrier breakdown in the sheep's brain post-stroke [5]. An extension of the Patlak plot model has been developed by Chen et al [76] and although not considered for use in this project, this model might provide a valid alternative to the extended Tofts model for this data. 


\subsection{Development of Curve fitting Techniques}

There were several variables to be considered in order to optimise curve fitting. This Section describes the methods undertaken to develop reliable, reproducible curve fitting methods and implement these in a computationally efficient way.

\subsubsection{Curve Fitting Algorithm}

The curve fitting package used in this project was from the Python library scipy and called the function optimize.minimize [77]. This function used the L-BFGS-B algorithm, described in full in [78].

A function was written that took a vector of the parameters as input, and output the sum of the squared errors between the fit with these parameters and the tissue concentration curve. By minimising the sum of squared errors using optimize.minimize, the values for the model parameters that best fit each tissue concentration curve were obtained.

\subsubsection{Initial Guess}

The curve fitting function used in this project required as input an initial guess of the parameters for the first iteration of curve fitting.

This initial guess proved an important parameter, as setting this guess too low or too high meant the fit might find a local minima rather than the set of parameters that minimised the entire fit.

A method that would have ensured a good initial guess for each voxel was iterating the initial guess for each voxel, and keeping the parameter values corresponding to the fit with the minimum sum of squared errors.

Due to computational restrictions, the iterative initial guess code in which each voxel was iterated through many initial guesses was not feasible. However, analysis of which initial guesses tended to produce better fits, and how much the fit changed depending on initial guess was undertaken. 
Analysis on initial guess setting was conducted on 7612 voxels from the DCE series of animal RT090 at 3 days post-stroke. This animal was chosen as it was the most severely affected animal from within the cohort, and so was likely to display the widest range of parameter values.

The initial guess for $K_{\text {trans }}$ was iterated over the values [0.0001, 0.001, $0.005,0.1]$, and the initial guesses for $v_{e}$ and $v_{\mathrm{p}}$ were iterated over [0.002, 0.02, $0.2]$. This iterative initial guess code resulted in 36 combinations of initial guess being tested.

For each voxel, the combination of parameters that minimised the sum of squared errors and the combination that maximised the sum of squared errors was output. The $R^{2}$ value corresponding to each of these combinations was output, and the average difference between the best and worst fit $R^{2}$ was found to be 0.14 . Excluding those voxels for which the best $R^{2}$ was less than 0.5 , the difference reduced to 0.11 .

This result indicates that in the worst case, in which the initial guess for each voxel is the worst possible combination of parameters for all voxels, the $R^{2}$ values of all fits would, on average, have an $11 \%$ error.

Taking the subset of 3124 voxels for which the best $R^{2}$ was over 0.5 , the difference between the average best and average worst estimate for the parameter $K_{\text {trans }}$, was found to be $60 \%$. The same average percentage difference between best and worse $v_{\mathrm{e}}$ was found to be more than $90 \%$, and for $v_{\mathrm{p}}$ was $7.5 \%$.

The initial guess for $v_{\mathrm{p}}$ was not further investigated, given the relative insensitivity of this parameter to changes in initial guess, and this value was set to be 0.02 .

It was found that $K_{\text {trans, } 0}$ of 0.1 was the best initial guess for $15 \%$ of the voxels sampled. $K_{\text {trans }, 0}$ of 0.0001 was the best guess for $35 \%$ of the voxels, and the initial guesses of 0.001 and 0.005 combined were the best options for the remaining $50 \%$ of voxels. The proximity of these guesses to eachother indicated that an initial guess for $K_{\text {trans }}$ between 0.001 and 0.005 would be appropriate for the majority of voxels, and so $K_{\text {trans0 }}$ was set to be 0.002 . 
For the parameter $v_{\mathrm{e}}$, the initial guess was set to 0.2 , the default value given in the software ROCKETSHIP, and further analysis was not conducted due to the model's insensitivity to this parameter, discussed further in Section 4.7.2.

The initial guess was therefore set to be [0.002, 0.2, 0.02], and this value was used for the results presented in later Sections.

\subsubsection{Treatment of Noise}

This Section covers methods involving both those voxels that produced a poor fit to the extended Tofts model, and the temporal oscillations seen in most tissue concentration curves across all animals.

\section{Treatment of poorly fit voxels}

At the fitting stage, all voxels were included and voxels that produced a poor fit were not excluded. Instead, all voxels within the region of interest were fitted regardless of the goodness of fit, and in analysis steps, values were excluded based both on sum of squared errors and of coefficient of variation of fit. These methods are further detailed in Section 6.2.2.

\section{Treatment of physiological 'noise'}

In the concentration data we see a periodic oscillation particularly following the uptake of the contrast agent.

While we might expect a curve displaying a high signal to noise ratio would display a smooth washout, the oscillations we see in the tails of each of the TCCs are representative of the natural dilation and constriction of blood vessels within the animal. In Figure 4.5 each time point is 5 seconds apart, and although we see periodicity in these oscillations of about $1 / 5 \mathrm{sec}^{-1}$, this signal is likely being biased and has a natural frequency higher than what we were able to detect with the 5 second temporal resolution. 


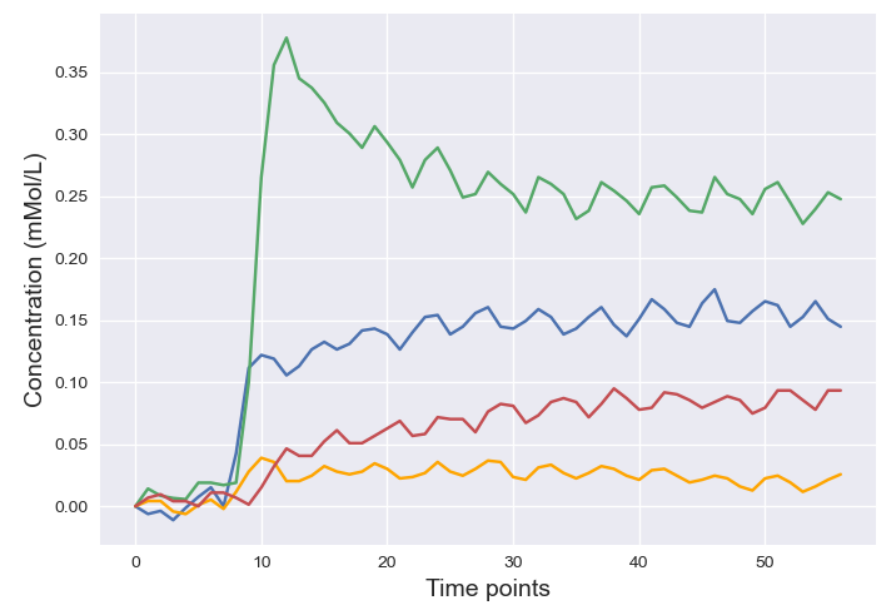

(a)

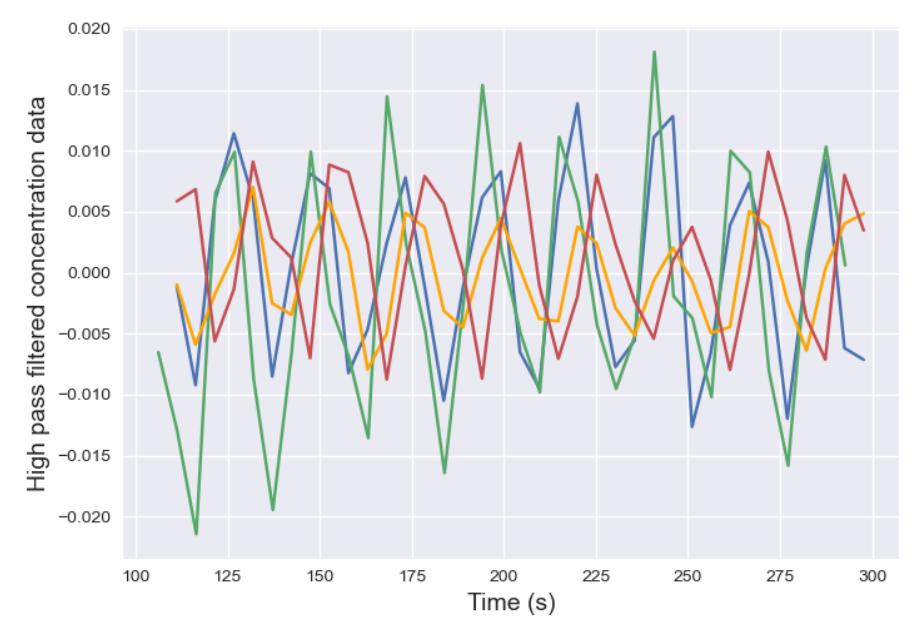

(b)

Figure 4.5: (a) Four example TCC's demonstrating physiological noise and (b) the same TCCs following high pass filtering to isolate the physiological oscillation component from the contrast washout curve. 
Although not technically noise, these periodic oscillations in the signal were considered as a potential source of error in curve fitting, as the pharmacokinetic models to be used did not include a term for these oscillations, and therefore the phase distribution of these oscillations throughout the image was investigated.

The AIF is representative of the concentration curve obtained furthest upstream of the arterial branches, which feed into the tissue of interest. As such, inclusion of the periodicity seen in the TCCs in the AIF would solve the issue of pharmacokinetic models not including this term, but only if the phase shift in these oscillations between TCCs in different regions of the image and the AIF was negligible. The steps taken to ensure that these oscillations would not be excluded in AIF selection are covered in Section 5.1.2.

In order to visualise phase shifts in these oscillations, a sample of 5 TCCs were selected from different places throughout an image and were high pass filtered using a first order Butterworth filter [79] with a cut off frequency of $120 \mathrm{~Hz}$ to reduce them to only the physiological oscillation component, as shown in Figure 4.5(b).

These values were then plotted against the high pass filtered AIF for the same image in order to visualise any phase shift between the AIF and the TCCs. The same values were then shifted incrementally by integer multiples of the time increment, 5 seconds.

As expected due to the temporal resolution of the images, the phase shift between the AIF and TCCs was minimised without any time shift applied.

\subsubsection{Computational Considerations in Fitting}

Performing a voxel by voxel fit of all voxels within the region of interest proved computationally expensive. Fitting times for a single animal frequently were 45 minutes to several hours, and given that there were 90 such images to fit, the limit of the processing power available on a single PC was quickly reached.

As such, the fitting code was rewritten to a multiprocessing capable file 


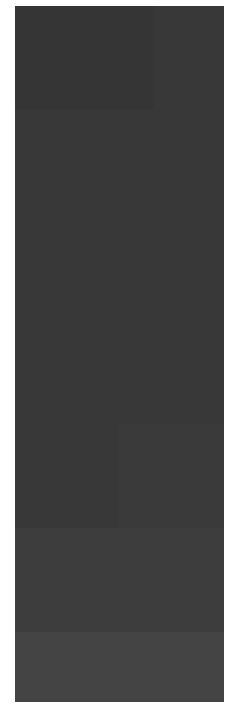

(a)

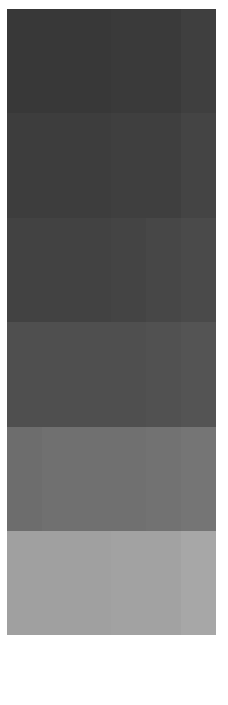

(b)

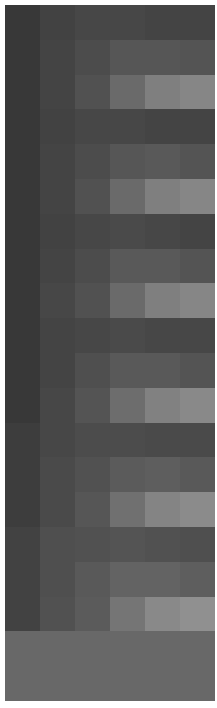

(c)

Figure 4.6: QIBA4 synthetic DCE-MRI data.

where a separate node in a high performance computing (HPC) cluster could fit each row and piece together the fitted image once all rows were fitted. Voxel by voxel fitting was therefore beneficial, as values calculated for one voxel were not dependent on values in surrounding voxels.

\subsubsection{Synthetic data verification of Curve Fitting Tech- niques}

In order to verify that the chosen fitting method was reliable and accurate, validation was performed on synthetic DCE-MRI datasets. These datasets were obtained from Daniel P. Barboriak's synthetic DCE-MRI dataset produced for the Quantitative Imaging Biomarkers Alliance (QIBA) [80].

In these synthetic datasets, the bottom $20 \times 60$ pixels represented a feeding artery. Above this, each $10 \times 10$ square of pixels was simulated to have a different set of parameters corresponding to the extended Tofts model. As shown in Figure 4.6, each of these $10 \times 10$ segments demonstrates different 
contrast uptake and washout characteristics, which resulted in different tissue concentration curves (TCCs) for each segment.

This concentration data was fitted to the extended Tofts model using both the Matlab plugin ROCKETSHIP and the python code written for this project in order to verify these methods were equivalent.

Parameter maps for $K_{\text {trans }}, v_{e}$ and $v_{\mathrm{p}}$ were generated and compared to the simulated values.

From Figure 4.7(a) and (c) we see that $K_{\text {trans }}$ and $v_{\mathrm{p}}$ values calculated using the python code written for this project match the simulated values well, as do the values calculated in ROCKETSHIP.

Due to being dependent on a good estimate for $K_{\text {trans }}$, the estimate of the parameter $v_{e}$ depends on the corresponding simulated value for $K_{\text {trans }}$. As such, in Figure 4.7(b) we see that the HPC and RS values for $v_{e}$ match well when the corresponding $K_{\text {trans }}$ value is above $0.05 \mathrm{~min}^{-1}$, but the estimate is unreliable when $K_{\text {trans }}<0.05 \mathrm{~min}^{-1}$. This unreliability in $v_{e}$ estimate is discussed further in Section 4.7.2, as the values for $K_{\text {trans }}$ estimated in this project were predominantly lower than $0.05 \mathrm{~min}^{-1}$.

Despite the inaccuracies in $v_{\mathrm{e}}$ estimate at corresponding low $K_{\text {trans }}$ values, the results of the python and ROCKETSHIP code provided confidence in moving forward with applying these fitting methods to the sheep images. 


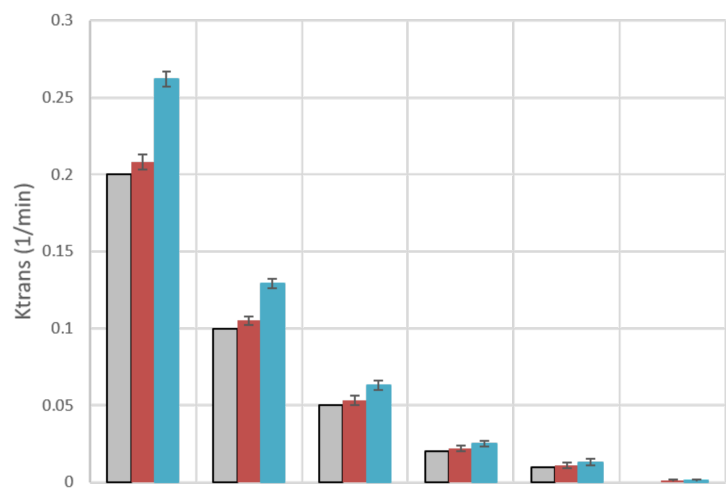

$$
\begin{aligned}
& \text { 口Ktrans simulated } \\
& \text { value } \\
& \text { - Ktrans HPC }
\end{aligned}
$$

(a)

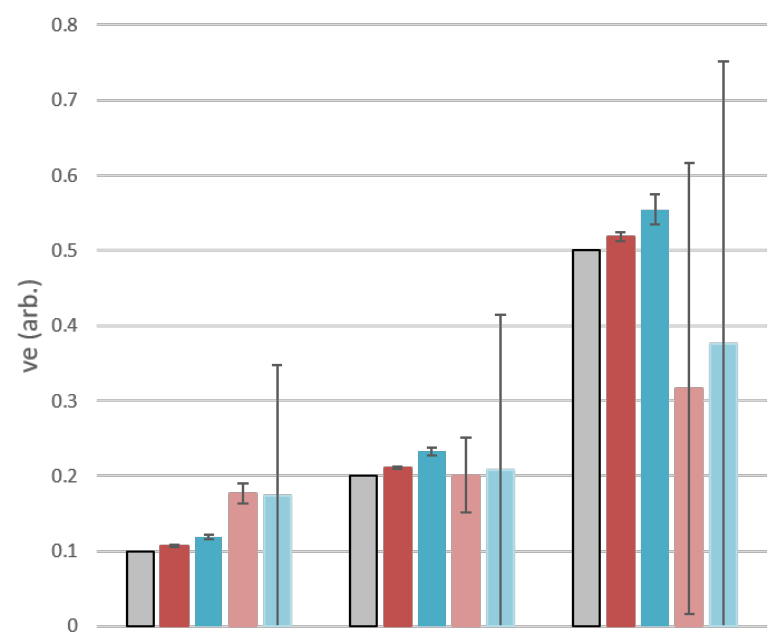

(b)

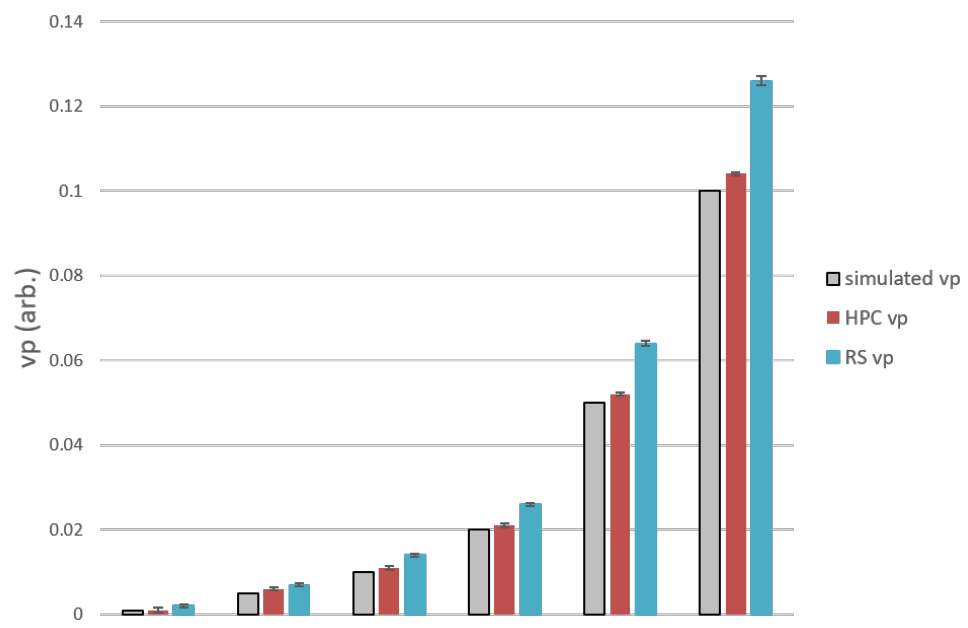

(c)

Figure 4.7: Comparison of synthetic data simulated parameters to values calculated through fitting in Python code (HPC) and in the Matlab plugin ROCKETSHIP (RS). 


\subsection{Discussion of DCE-MRI Analysis Exper- imental Methods}

The methods presented in this Section demonstrate the varied range of approaches that were required in extracting kinetic parameters from the DCE data, including the selection of pharmacokinetic model, the validation of $T_{1,0}$ map methods, determination of the best initial guess for fitting and consideration of physiological noise. Discussion of the methods presented in this chapter, and consideration of future improvements to these methods is presented here.

\subsubsection{Discussion of $T_{1}$ map generation}

A concern in the generation of $T_{1}$ maps in this project from the dual flip angle images was that if any machine settings, such as reciever gain, were altered between the acquisition of the two images, the assumption that $S_{0}$ remained unchanged between the 2 and 25 degree flip angle images would be incorrect.

A representative from Siemens was contacted and confirmed that the parameters identified from the DICOM tags as changing between acquisitions were related to the number of phase encoding steps rather than receiver gain settings. The 2 degree image uses eight averages, while the 15 degree uses six averages.

Differences in the RF excitation voltage between the 2 and 15 degree images were also identified, however these differences were identical to the default Siemens library sequence values and so were not considered as a potential source of error in $T_{1}$ mapping from these dual flip angle images.

In order to confidently verify the $T_{1}$ map generation method used in this project, running the same dual flip angle and DCE protocol on a look up table based phantom [65] containing several different samples with known $T_{1}$ times would be ideal. This would allow for verification of both $T_{1}$ maps, but also provide confidence in the $R_{1}$ values calculated off DCE images, and of 
the expected range of values for concentration of contrast agent.

As this data was not available for this project, validation was performed through analysis of images and independent experiments conducted on the 9.4T system available at Victoria University of Wellington, and a second cohort of animals imaged at SAHMRI in Adelaide in 2020.

\subsubsection{Discussion of Curve Fitting Techniques}

\section{Sensitivity of sum of squared errors}

As mentioned in this section, the sensitivity of the extended Tofts model to changes in each parameter was not equal between the three perfusion parameters under consideration.

In order to investigate the sensitivity of the model to changes in each parameter, the sum of squared errors was calculated as a function of each perfusion parameter.

Several of these Figures are shown in Figure 4.8. Figure 4.8(a) is the finding which most influenced future decisions in experimental techniques. This plot shows that as $K_{\text {trans }}$ approaches 0 , the estimate of $v_{\mathrm{e}}$ becomes less and less sensitive, to the point when $K_{\text {trans }}=0, v_{\mathrm{e}}$ can not be estimated.

The effect of the $v_{\mathrm{e}}$ estimate's dependence on $K_{\text {trans }}$ can be understood from the extended Tofts model impulse response function, reproduced here:

$$
C_{\mathrm{t}}(t)=K_{\mathrm{trans}} e^{\frac{K_{\mathrm{trans}} t}{v_{\mathrm{e}}}} * C_{\mathrm{p}}(t)+v_{\mathrm{p}} C_{\mathrm{p}}(t)
$$

In the case where $K_{\text {trans }}=0$, no information can be gained about $v_{\mathrm{e}}$. Physiologically, this can be interpreted as for an intact vessel with effectively zero permeability, where $K_{\text {trans }}=0$, the contrast agent will remain in the plasma space only and no leakage to the interstitial space will occur. As such, we can not know the fraction of tissue that is interstitial space when $K_{\text {trans }}$ $=0$, and this effect continues at low values of $K_{\text {trans }}$ in the form of a broad estimate of $v_{\mathrm{e}}$ only. 
In terms of sum of squared errors, which is the optimisation criteria for curve fitting in this project, this means that a wide range of $v_{\mathrm{e}}$ values will be equivalent to eachother at lower values of $K_{\text {trans }}$.

This statement does not hold for the parameter $v_{\mathrm{p}}$ or $K_{\text {trans }}$ to the same extent. Figure 4.8(a) shows as the true value for $K_{\text {trans }}$ increases, the sum of squared errors for $K_{\text {trans }}$ becomes more and more permissible of a wider range of values. In this project, the values for $K_{\text {trans }}$ were expected to be within the lower range for which the sum of squared errors remained relatively precise.

Figure 4.8(c) shows the effect of the parameter $v_{\mathrm{e}}$ on estimates of the same true value of $K_{\text {trans }}$. Compared to Figure 4.8(a), this estimation, even for the lowest value of $v_{\mathrm{e}}$, remains relatively precise.

Finally, the estimate for $v_{\mathrm{p}}$ is unaffected by estimates for $K_{\text {trans }}$ and $v_{\mathrm{e}}$, as shown in Equation 4.13, the estimate of this parameter depends only on the AIF estimate.

In order to exactly quantify the sensitivity of the sum of squared errors to changes in each parameter, further investigation involving the Cramér-Rao bound could be undertaken [81].

\section{Discussion of Initial Guess}

Setting of the initial guess was an analysis step that took significant investigation into how much the initial guess could impact fit results, and to determine the best method of setting initial guess in this project.

Although it would have been preferable to iterate every voxel through a range of initial guesses, the results on synthetic data indicated that for some voxels, this would not always have resulted in an improved parameter estimate. An iterated initial guess was conducted on QIBA synthetic data [80] and the results are shown in Figure 4.9. 


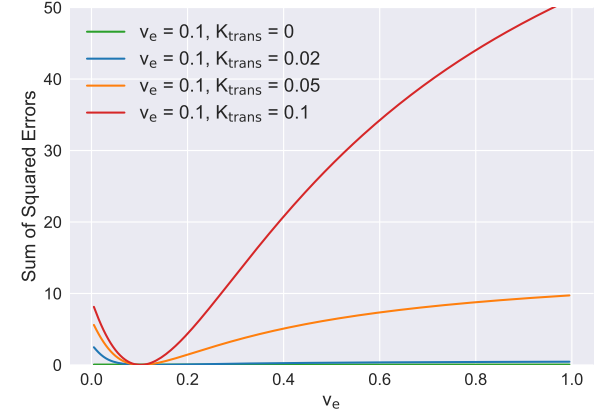

(a)

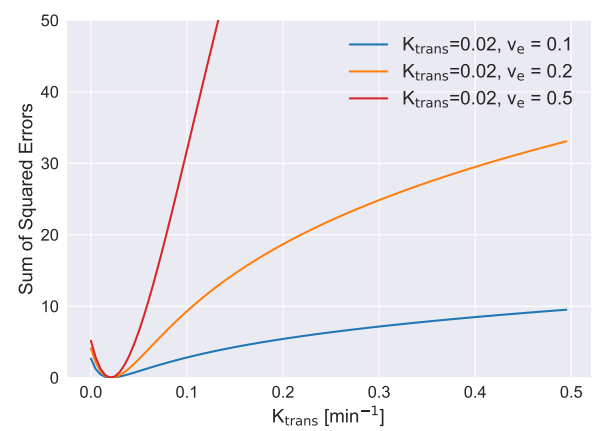

(c)

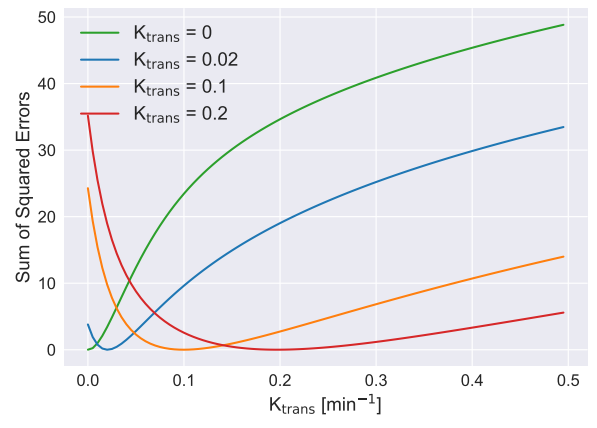

(b)

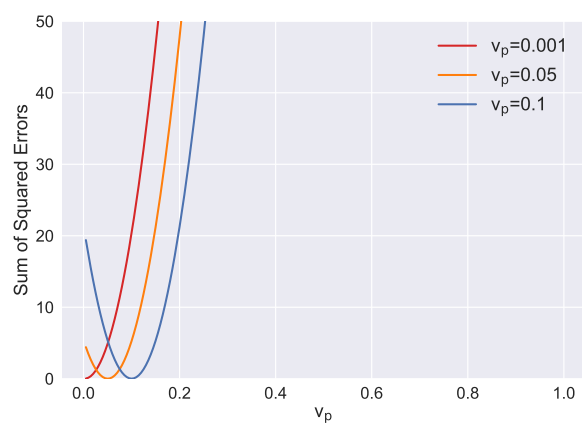

(d)

Figure 4.8: (a) sum of squared errors of $v_{\mathrm{e}}$ with different values of $K_{\text {trans }}$ (b) sum of squared errors of $K_{\text {trans }}$ with different values of $K_{\text {trans }}$ (c) sum of squared errors of $K_{\text {trans }}$ with different values of $v_{\mathrm{e}}$, (d) sum of squared errors of $v_{\mathrm{p}}$ with different values of $v_{\mathrm{p}}$ 


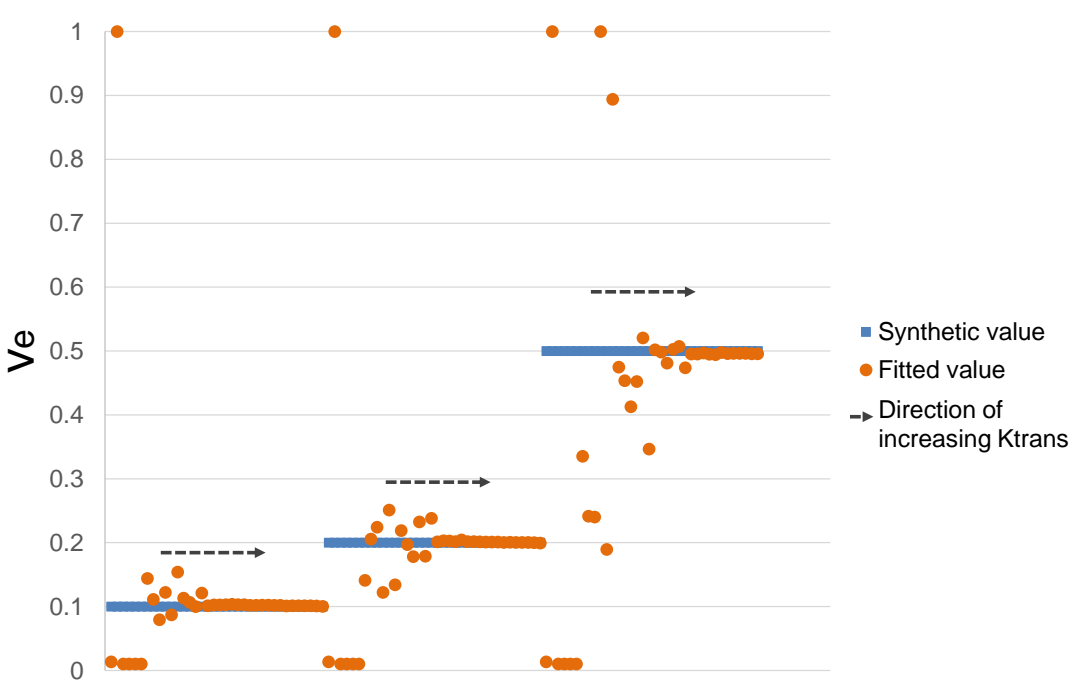

Figure 4.9: Comparison of synthetic $v_{\mathrm{e}}$ value with fitted value, computed with iterated initial guess.

\subsubsection{Exploration of existing software packages}

The multitude of image processing steps described in this project meant that fully processing a single animal from DCE data to perfusion parameters was a time consuming and computationally expensive process. The methods used in this project were not chosen to reduce time spent on each animal, as this entire thesis was based around developing best techniques for the processing and analysis of this particular DCE-MRI dataset. Instead, future research could focus on exploring usage of existing DCE-MRI processing software, such as ROCKETSHIP [60], which was used for part of this project, or other software packages such as dcemri.jl [61].

The downsides of these software packages included

- user interaction was required at some/all processing steps, which was not feasible for $90+$ image series.

- any code customisation (such as implementing an iterated initial guess or custom AIF selection tool) required learning the programming language 
of the respective software package (e.g. julia for dcemri.jl)

- data needed to be in different formats for different software packages (e.g. NIfTI and MAT files for ROCKETSHIP)

As such, in this project, keeping all code in the same language and files in the same format proved more valuable in saving time and computational resources than adjusting the experimental methods to be inline with an existing software package.

\subsubsection{Summary of DCE-MRI Analysis Methods}

In this Section we have seen methods for reliable $T_{1,0}$ map generation from dual flip angle $T_{1}$ weighted images, comparison of this method to inversion recovery and three flip angle $T_{1}$ map generation, a method for conversion from DCE signal to concentration, curve fitting techniques, and validation of these methods against synthetic DCE-MRI data. As the first research to analyse these DCE-MRI datasets, care was taken at each step to ensure best possible approaches were being undertaken, and to ensure the generated perfusion maps were a reliable foundation for analysis detailed in the following Sections. 


\section{Chapter 5}

\section{AIF Selection Methods}

Selection of one AIF over another can greatly impact the results of the extended Tofts model. The importance of best AIF selection in robust parameter determination therefore cannot be overlooked.

The gold standard in AIF estimation involves drawing blood from the subject throughout the contrast injection and image acquisition, and analysing the contrast agent concentration within each blood sample [39]. Obtaining a gold standard AIF from each animal would evidently have been a highly involved process, and was not feasible in these animal studies. Each animal also displayed vastly different responses to the surgical procedure, implying that even if a gold standard AIF had been obtained from a single animal, this AIF would not be representative of the inter-animal differences in cerebral contrast agent uptake.

As the gold standard AIF measurement method described here is an invasive and time consuming process, there exist many alternative methods of estimating an AIF.

These include:

- Using an automated search to return an AIF of the desired shape from the image data [35]. The algorithm used in this project is described in Section 5.1.2. 
- Selection of an AIF based on knowledge of the subject anatomy. Application of this method is covered in Section 5.2.

- Using an averaged population based AIF [82]. This method was not feasible in this project due to the lack of published data measuring gold standard AIFs in sheep.

- Blind estimation from the tissue concentration curves [83]. This method was not able to be successfully implemented in this project, but the methods attempted are described in Section 5.4.2.

The goal of each of these AIF estimation methods was to determine the voxel(s) from which the best AIF estimate could be selected. In order to compare one potential AIF candidate to another, we first must understand the desirable characteristics of an AIF. Following this, several of the AIF selection methods were implemented and the best AIF estimates for each animal on each day were determined.

\subsection{AIF Selection Algorithm}

\subsubsection{Desirable AIF Characteristics}

With knowledge of the physiological requirements for an AIF, analysis of tissue concentration curves can determine if a curve is a good AIF estimate.

As the AIF estimate is designed to model input of a contrast bolus feeding into surrounding tissue, it is evident that compared to signal enhancement curves from within the local tissue of interest, the AIF should demonstrate the earliest signal enhancement and the highest peak concentration.

These characteristics can be measured from the concentration time curves in terms of the time to peak $\left(t_{\max }\right)$ or bolus arrival time $(\mathrm{BAT})$, and the concentration curve maximum. We also expect the AIF concentration to drop rapidly following the first peak as the bolus passes through the artery without perfusing into the surrounding tissue. This will result in a narrow 


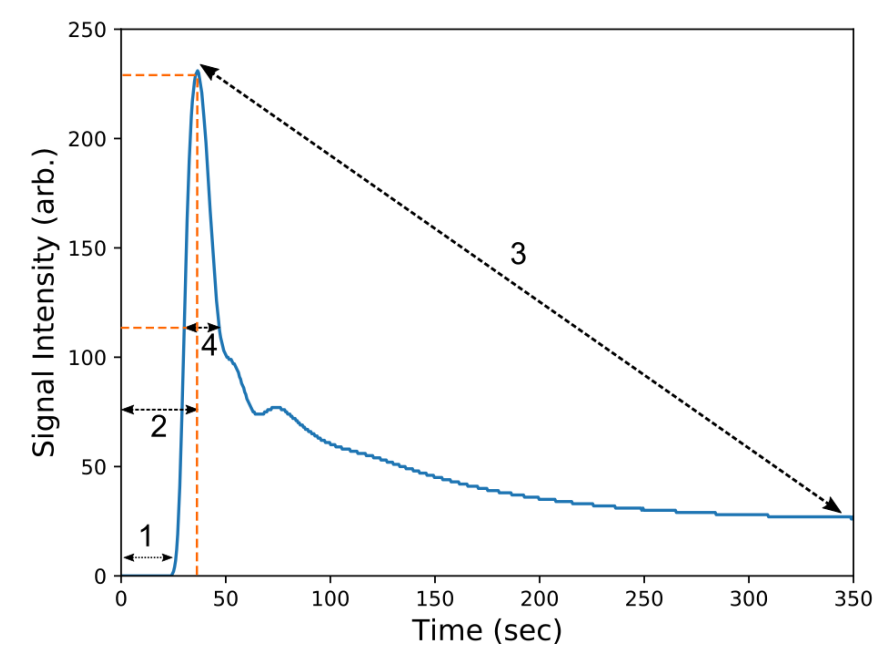

Figure 5.1: A synthetic, ideal AIF generated using the programme JSim [84] showing 1 . bolus arrival time 2. time to peak 3. washout slope 4 . full width half maximum

peak, which can be quantified from the full width half maximum (FWHM) of the first pass peak, or the wash in and wash out slopes.

\subsubsection{AIF Search Criteria}

While in synthetic data, it is simple to implement a search for the voxel with the best values for each of these semi-quantitative measures, for the sheep data, several factors made direct calculation of some of these parameters, such as bolus arrival time or FWHM, impractical.

Physiological noise, the treatment of which is discussed further in 4.6, frequently masked the wash out slope values, and the difference in bolus arrival time in the arteries and veins could not be distinguished due to temporal resolution, discussed previously in Section 4.5.2.

Instead, an algorithm was developed to find the best AIF candidate based on the criteria described in Section 5.1.2 


\section{Algorithm Pseudocode}

This Section provides a simplified description of the AIF selection algorithm steps.

1. Apply chosen mask to look only within certain anatomical region of interest.

2. From within these voxels, return the concentration curve with highest peak concentration

3. ...where the peak concentration occurs within the injection time frame

4. ... and the standard deviation of points following the peak is below a user set value (used 0.15 to neglect noise voxels, but include physiological oscillations. Discussed further in Section 5.4.2.

5. ... and the end signal drops by user set percentage of maximum value.

\subsection{Anatomy Based AIF Selection}

Along with the DCE-MRI series for each animal at each time point, there was a wealth of other physiological information available within the various other image data sets taken as part of the MRI protocol. These include: $T_{2}$ weighted images, diffusion weighted images and angiography images. Of particular interest in AIF selection were the angiography images, as these images are designed to clearly highlight vasculature, which could provide useful information for determining the location of any feeding arteries.

\subsubsection{Anatomical Masks}

In order to narrow down AIF search to physiologically relevant areas, a binary image mask was created. By applying a mask to an image reduced region in which an AIF search was performed, thus significantly reducing computational 


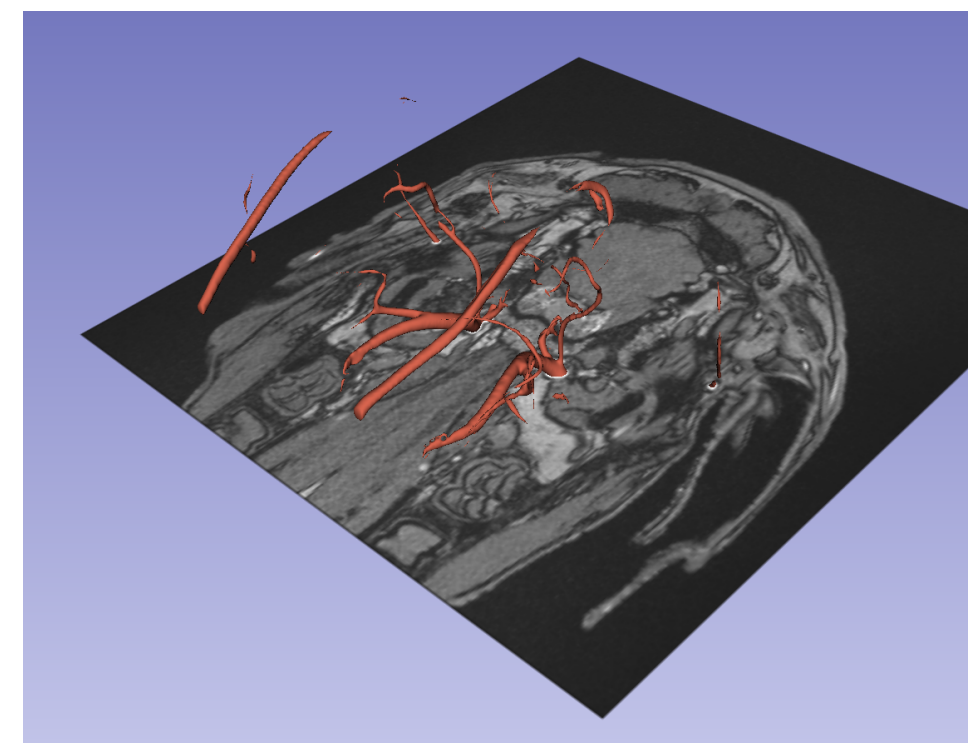

Figure 5.2: 3D representation of MRA thresholded mask highlighting vasculature within the anatomy for animal RT107 at 24 hours poststroke.

time and improving quality of an AIF estimation. In this project there were two methods of anatomical mask creation, both using the program 3D slicer.

\section{MRA Thresholded Masks}

The first type of mask generated in this project used the MRA images and involved segmenting through thresholding of the image. This process involved setting a value above which all voxels were included in the mask. Due to the nature of the MRA images, this resulted in the segmentation of all major arteries and veins in the image, represented in 3D in Figure 5.2.

\section{Manual Anatomical Segmentation}

The second type of segmentation involved manually painting in areas to be included in a mask. This method was time consuming, but proved the most reliable for segmenting out large areas such as the left and right hemisphere 


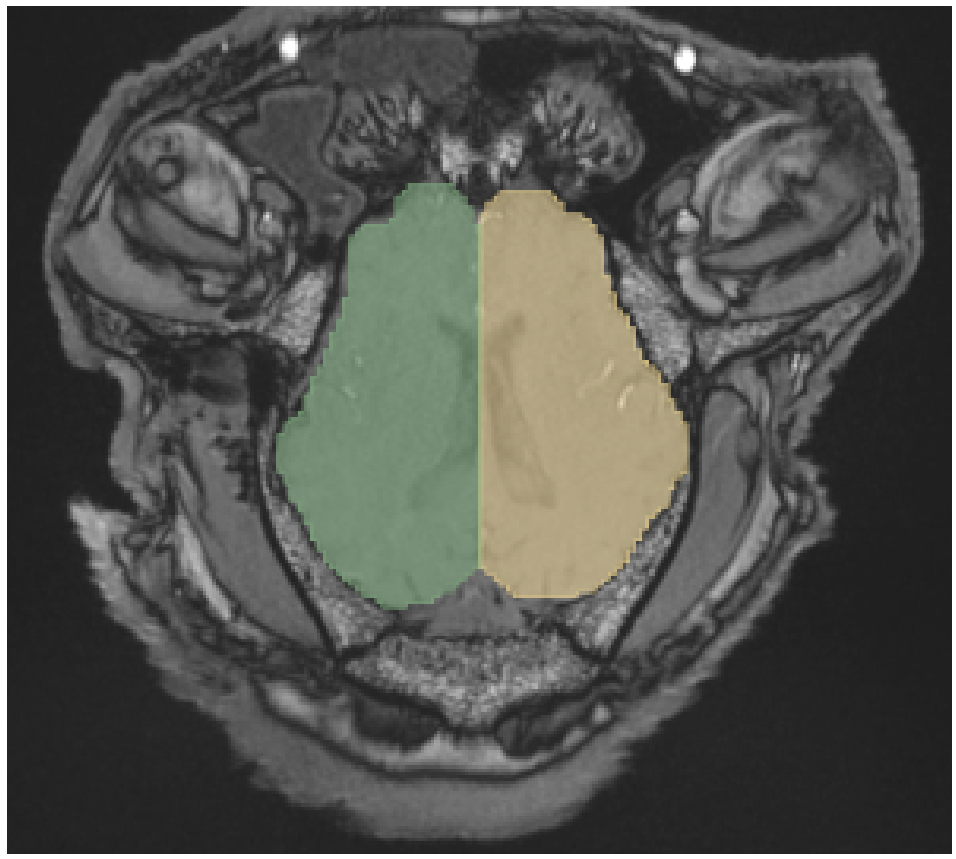

Figure 5.3: Screen capture of a manually segmented slice of animal RT107.

for each sheep. Following manual segmentation of 3-4 slices, the remaining slices could be automatically segmented using the Fill Between Slices function in 3DSlicer [64]. This resulted in a semi-automated hemisphere segmentation process which was used for all animals at all time points.

\subsubsection{Three Test Anatomy Based Masks}

While the mask extracted from the MRA images clearly segments out vasculature, this alone was not ideal for AIF selection due to several factors.

Firstly, this mask included arteries and veins- including the large sagittal sinus. The inclusion of this vein meant that applying the automated search to this area often returned AIFs that were actually from a vein rather than the internal carotid artery. At this stage, AIFs from veins were rejected as invalid, however as discussed in Section 4.5, the temporal resolution meant 
that there could have been an argument to be made for selection of the large sagittal sinus as an AIF, and this technique has been described in literature for the case where temporal resolution means arterial input and venous output are temporally indistinguishable [37]. While the bolus arrival times between arterial input and venous output in this data were indistinguishable, AIF searches in this project were still restricted to only arterial regions due to the uncertainties in bolus dispersion shape that may be introduced using a venous output as an AIF.

Secondly, due to the thresholding applied, the MRA mask selected only the very central portion of vasculature, which would represent the fastest flowing plasma in the cross section, most likely to contribute to partial volume effects [85].

Motivated by the fact that the MRA mask alone may not be ideal for AIF selection, three potential AIF selection masks were created and tested to see which anatomical segmentation yielded the best AIF. This testing process involved applying each type of mask to the prestroke and 6 day DCE image series from a representative subset of four animals, and visually assessing the AIFs detected from each.

\section{Brain Hemisphere Mask}

The first mask under investigation as a potential AIF selection mask was the whole brain mask, manually segmented for each animal on each acquisition day.

The benefit of this mask was that for a local AIF, it is preferable to select from within the tissue of interest, in this project- the left and right brain hemispheres. The results of applying this mask with the algorithm described in Section 5.1.2 are given in Figure 5.4.

While the tissue of interest in parameter value calculations was fully captured by the hemisphere mask, this mask did not always capture the feeding arteries which are located at the base of the brain. This mask also did not exclude veins such as the posterior sinus, and as such some of the 
curves displayed in Figure 5.4 may have been selected from veins.

\section{Rough Artery Segmentation Mask}

The second mask was a wide segmentation over the location of the arteries at the base of the brain. This mask is referred to in this project as a rough artery segmentation, as while it would likely cover the arteries in each of the animals, a new mask was not made for each animal at each time point, and the mask itself covered a large area around each artery.

\section{Dilated MRA Mask}

The third mask that was tested began with using the initial MRA mask, but after applying a binary dilation process in ImageJ [62].

The motivation for testing this mask was that dilating the MRA mask would mean the mask would be able to capture more of artery cross section than simple thresholding.

\subsubsection{Test AIF Mask Results}

Using the algorithm described in Section 5.1.2, each of the three AIF mask candidates were tested on a subset of animals with a range of infarct sizes. (RT090, RT102, RT104 and RT109 from Table 8.3). The results of this test are shown in Figure 5.4.

There are a few points to note from this Figure. The first is in the whole brain mask for the prestroke animals, Figure 5.4(a), we see a wide variation in AIF peak value and shape, whereas the peak concentration value across the different animals appears more similar in both the dilated MRA mask and the rough artery segmentation.

The second is the case of the prestroke animal RT090, shown in red. In 5.4(a), it appears that a better AIF has been found than in 5.4(b) or (c). However, upon investigation of where this AIF was selected from, shown in Figure 5.5 we see that this is in fact a venous output rather than an AIF. As 


\section{Whole Brain Mask}
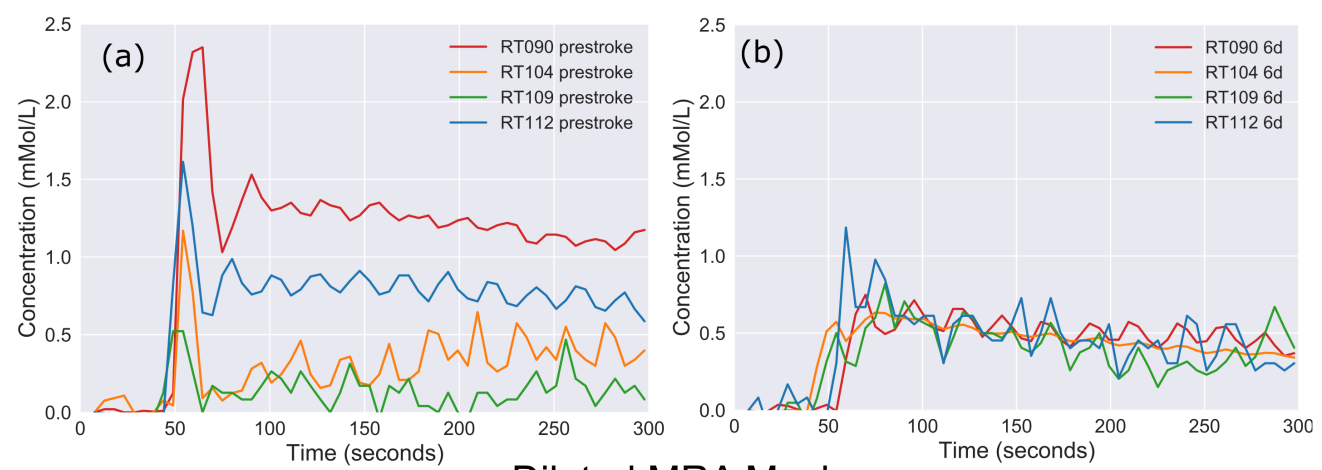

Dilated MRA Mask
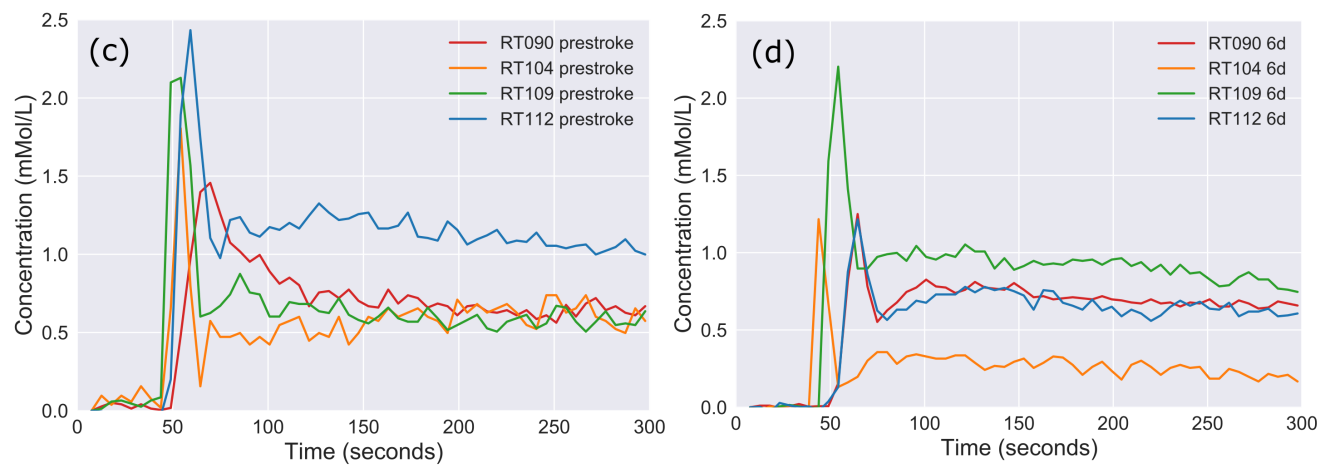

\section{Rough Artery Mask}
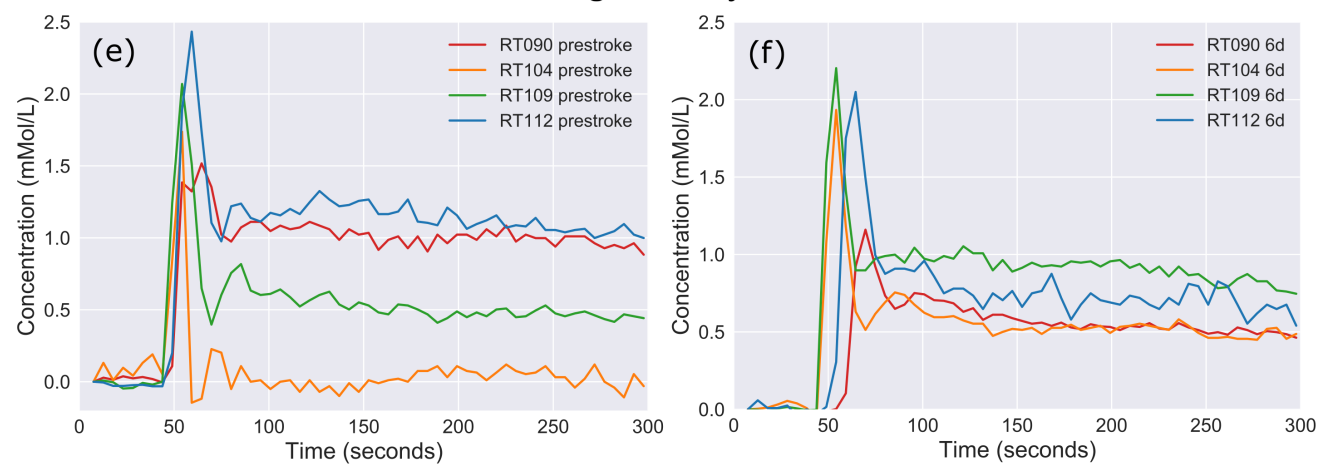

Figure 5.4: Results from AIF search within (a) whole brain mask in healthy animals (b) whole brain mask in $6 \mathrm{~d}$ post-stroke animals, (c) dilated MRA mask in healthy animals (d) dilated MRA mask in $6 \mathrm{~d}$ post-stroke animals (e) rough artery mask in healthy animals (f) rough artery mask in $6 \mathrm{~d}$ post-stroke animals. 


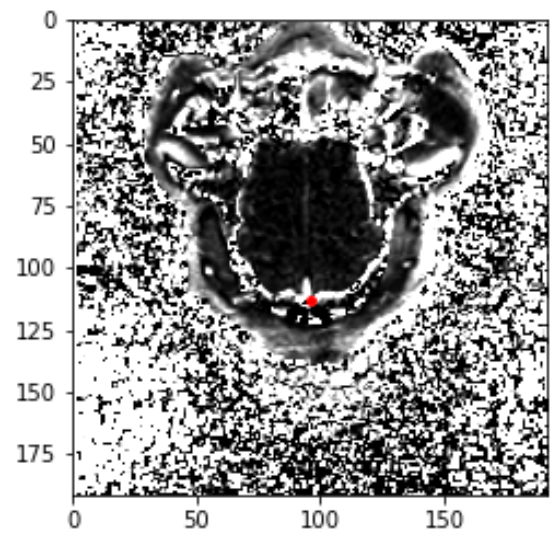

(a)

Figure 5.5: Algorithm found best AIF location for animal RT090 using the whole brain mask. This area is the sagittal sinus, a large vein that drains blood out of the cerebral parenchyma rather than feeding in.

such, while the RT090 AIF in both 5.4(e) and (f) has a lower peak value than that found in (a), these lower peak value AIFs are from a more physiologically sound location. 5.4.2

Upon comparing 5.4(d) and (f), there is little difference in found AIFs, which is expected given these masks cover similar regions of the brain. However, the rough artery segmentation mask appears to detect higher peak value AIFs for animals RT104 and RT112, likely due to the broader segmentation associated with this mask. As such the rough artery segmentation mask was chosen for use in further image processing.

\subsection{Physiological Validation of AIFs}

One of the established methods for AIF selection from DCE images is enlisting a specialist, i.e. a radiologist or similarly trained professional, to determine 
from the anatomy where in the image would be an appropriate AIF selection point. In this project, this exact method was not feasible due to the differences in human and sheep anatomy, and the total number of images for which an AIF estimate was needed $(90+)$.

Following selection of semi-automated AIFs, images highlighting the selected voxel for each AIF were shown to a subject expert (A. J. SorbyAdams, personal communication, 13th April, 2021) and the anatomy from which the AIFs was selected was verified to be the internal carotid artery (ICA). The ICA was confirmed to be the ideal location in the sheep's anatomy from which to select a global AIF, as this location represents a feeding artery to the brain, and is upstream from the location of the transient occlusion in the middle cerebral artery.

\subsubsection{Preparation for further analysis}

Following generation of all AIFs, the final step before curve fitting could begin was conversion of the AIF from blood concentration $C_{\mathrm{b}}$, to plasma concentration, $C_{\mathrm{p}}$.

This step is required to account for the fact that the contrast agent is carried only in the plasma within an artery, and so the concentration of the contrast agent in the plasma of an artery is higher than in the whole blood. This conversion from blood to plasma concentration used a simple equation:

$$
C_{\mathrm{p}}=\frac{C_{\mathrm{b}}}{(1-\mathrm{HTC})}
$$

where HTC is the sheep specific haematocrit such that the $1-$ HTC term isolates the plasma fraction of the blood. The value for ovine haematocrit was determined from literature to be 0.28 [86].

Following AIF detection, concentration map generation, and conversion of AIF to $C_{\mathrm{p}}$ all data analysis and preparation was complete and extended Tofts model fitting could begin. 


\subsection{Discussion of AIF selection methods}

Decisions regarding AIF selection methods in this project were made in order to optimise AIF selection specifically within this dataset. For different areas of the anatomy, or for images with an improved temporal resolution, different steps could be taken to optimise AIF selection. An evaluation of strengths and weaknesses of the chosen AIF selection methods in this project is presented here, as well as potential alternative methods that could be used in future research.

\subsubsection{Discussion of AIF selection algorithm}

The AIF selection algorithm used in this project relied on both the anatomical mask and the AIF selection criteria, and the chosen mask was a rough segmentation around both the left and right internal carotid arteries. This mask, however, was not specific to each animal and as such included a wide area of pixels around the internal carotid artery in each image. Ideally, the internal carotid artery would be manually selected in each image and an ROI placed within it for AIF selection. In this project, this was not a possibility due to the number of images that required AIF segmentation (90+) and the availability of experts on sheep anatomy. Instead, the mask used was designed to be broad enough to include both the left and right ICA in each animal, and the final location of AIF selection was output as an image which was then verified as being from a sensible physiological location by an expert in this subject (A. J. Sorby-Adams, personal communication, 13th April, 2021).

Since the anatomical mask applied was designed to be a broad artery segmentation, selection of an AIF voxel was then determined by the algorithm's selection criteria.

The criteria included in the algorithm were time to peak, standard deviation of washout tail, and an interpretation of the washout slope in the form of percentage drop between the peak and the final concentration. 


\section{Percentage concentration drop}

This percentage was set to be $60 \%$, such that AIF voxels were only considered if the final concentration had dropped by at least $40 \%$ of the peak signal. $60 \%$ was used as the initial threshold, and following visual evaluation of the selected AIF, this threshold was relaxed as needed if a suitable AIF meeting this criteria could not be found.

\section{Washout standard deviation}

The criteria of standard deviation of washout peak values proved important in filtering out noise voxels, but this value needed to be set so that it would not preferentially exclude tissue concentration values with overall higher concentration values- as high peak concentration was a desirable AIF characteristic. This value also could not be set so low that the physiological oscillations described in Section 4.6.3 were excluded, as it was desirable for the AIF to retain these oscillations for later curve fitting.

\section{Animals with no suitable AIF}

In the case of some animals, no visually suitable AIF could be found. In these cases, the algorithm and mask were adjusted in order to widen the search scope but either the selected AIFs were too noisy, had too low peak concentration relative to the peak values in surrounding tissue, or would be otherwise visually unsuitable. Examples of these AIFs are given in Figure 6.2 in the following Chapter, as these AIFs were excluded at the analysis stage.

The first alternative to exclusion for an animal with a visually unsuitable AIF was to average the remaining AIFs at each time point. This method, however, introduced issues with selecting of a synthetic bolus arrival time. As the remaining AIFs were estimated from different acquisition days, the variation in bolus arrival time between days meant that in order to average these AIFs a phase shift needed to be introduced to each to align the peak concentration values and washout tails. In order to do this, AIFs were 
synthetically shifted in time and padded. Once averaged, however, the actual bolus arrival time for the AIF was unknown. In this dataset, the difference in bolus arrival time between venous and arterial tissue concentration curves was generally negligible, however the averaged AIF would need a bolus arrival time.

Another alternative to averaging an AIF that was explored was blind AIF estimation from parameter values, which is discussed in the following Section as an option for AIF selection in future research using this dataset.

\subsubsection{AIF Selection Improvements for Future Research}

\section{AIF Blind Estimation}

The method explored was adapted from the methods presented in [83], to simultaneously estimate parameters and AIF from voxels. These methods are described fully in [83], but, briefly, involved:

1. Select a population based AIF as an initial guess.

2. Estimate the perfusion parameters by fitting a subset of TCCs with this initial AIF guess.

3. Calculate the tissue impulse response function for each TCC corresponding to these parameters.

4. Update the AIF initial guess to the deconvolution of the tissue concentration curve and the impulse response function for each TCC, and average this result to obtain a new AIF estimate based on the impulse response functions of all TCCs.

5. Refit the TCCs with the new updated and averaged AIF estimate and obtain new parameter estimates.

6. Update the tissue impulse response function with the new parameter estimates. 
7. Steps 4-6 are repeated until AIF estimate converges.

This method was appealing as it offered simultaneous parameter and AIF estimation, was an objective AIF detection method not relying on subject specific visual interpretation of the AIF, and also did not require that a suitable AIF voxel was present in the image.

Preliminary investigation was conducted to investigate this method as an AIF selection option in this project, using the Schabel-Parker population based AIF as the initial guess for Step 1 [87]. The AIF estimate was determined to have converged if the sum of squared errors between subsequent estimates fell below a set value $\left(1 \times 10^{-6}\right.$. Following this, the AIF estimate was output, and this process was repeated for all animals.

There were several issues that arose in this preliminary investigation that meant this method was not pursued further in this project. The first was that the AIFs generated using this method failed to reproduce reasonable parameter estimates once fit to an entire image, and had peak concentrations much higher than expected with knowledge of the the contrast agent dose. This was perhaps due to the population based AIF used for the initial guess, which was based on human uptake of contrast agent in breast cancer patients [82]. This may have been solved by normalisation of the AIF at each step, allowing for estimate of the shape of AIF alone but not the peak value. However, this was not desirable in this project, as an empirical measure of peak concentration at the feeding artery was not available.

A second issue with this method was that it was not entirely blind in its application, and required prior knowledge of which TCCs were good candidates for inclusion in the sample for fitting. In this investigation, the TCCs included in the sample were selected with prior knowledge of coefficient of variation ( $\mathrm{R}^{2}$ from model fitting with the animal specific AIFs.

While a promising method for simultaneous AIF and parameter estimates, application of this method remains an area of future investigation for analysis of this dataset. 


\section{Physiological Noise in AIF Selection}

An option for AIF selection methods in future research using this dataset could be to include a term in the model used to fit that accounts for the physiological oscillations, and then smoothing could be applied to voxels prior to AIF selection, allowing for a lower standard deviation threshold to be set. Altering existing pharmacokinetic models of perfusion was, however, outside the scope of this project.

\section{BAT Based AIF Selection}

In research using datasets with a higher temporal resolution, a criteria for AIF selection that was not applicable in this project would be bolus arrival time (BAT). A recent paper has shown that this BAT is a good AIF selection criteria for both local and global AIFs [88], however in this project this was not feasible due to low temporal resolution.

\subsubsection{Summary of AIF selection methods}

The method for AIF determination in this project involved an anatomical mask segmenting out a broad region around the left and right internal carotid arteries, and application of an AIF selection algorithm that selected the highest peak, with sufficiently low standard deviation of washout slope, and that dropped to a set percentage of the concentration peak by the end of the acquisition. Several AIF selection methods were attempted, including using different anatomical masks, blind AIF estimation, and using existing software packages. The AIF method eventually selected for use in this project reliably produced visually acceptable AIFs for the majority of animals from a physiologically sound location, although for some images a suitable AIF voxel could not be found, leading to exclusion of some images from consideration in later Sections. Due to the effect the AIF estimate has on the estimated parameters, the development of the methods detailed in this Section proved to be one of the most important steps in this project. 


\section{Chapter 6}

\section{Results and Analysis}

The goal of the analysis presented in this chapter is two-fold. The first is simply to quantify the perfusion changes we see in an animal's brain as a result of the stroke. This takes the form of comparing perfusion measurements over time, and in the infarcted hemisphere to the opposite hemisphere over time. Secondly, this analysis strives to isolate the perfusion changes resulting from only the induced stroke, and reject influences from confounding factors such as ventricle and vasculature signal, noise, outliers, choice of mask and other unforeseen experimental difficulties of working with an animal model.

The first part of this Chapter reports hemisphere level analysis, conducted to obtain global, high level parameter changes following stroke. Following this, parameter histogram level analysis is presented, which allowed for investigation into the value distribution of $K_{\text {trans }}$ within each hemisphere. Finally, due to the heterogeneity of the ischemic tissue in each hemisphere, small ROI based analysis was conducted in order to isolate voxels from areas of cytotoxic and vasogenic edema, and compare the changes in perfusion parameters from within these areas. 


\subsection{Perfusion Maps}

Results presented in this Section were obtained from fitting the extended Tofts model to the concentration maps generated according to the procedure outlined in Section 4.6. A unique AIF was generated for each animal on each acquisition day using the rough artery anatomical segmentation described in Section 5.2.3 and the AIF detection algorithm described in Section 5.1.2.

Figure 6.1 displays perfusion parameter maps for animal RT090, which demonstrates one of the most clearly visible infarcts of all animals processed in this project. Increases in both $K_{\text {trans }}$ and $v_{\mathrm{p}}$ can be observed, particularly in the 6 day post-stroke image. Another feature of these images is the vasculature, which is the brightest feature of the $v_{\text {p }}$ perfusion maps at each time point. In the prestroke $K_{\text {trans }}$ these two symmetrical lobes are instead dark areas, indicating that the highest plasma volume in the prestroke $v_{\mathrm{p}}$ map corresponds to a low $K_{\text {trans }}$ area. According to the extended Tofts model, we would expect vasculature with intact blood vessel wall, in this case the blood brain barrier, to have $K_{\text {trans }} \approx 0$, which is consistent with the prestroke findings.

Finally, the $v_{\mathrm{e}}$ maps give very little information. At 6 days we see a small region of decrease corresponding to the ischemic tissue (as determined from the $K_{\text {trans }}$ map), whereas the remaining area of the $v_{\mathrm{e}}$ map gives no information. This is likely due to the relative insensitivity of the model to changes in $v_{\mathrm{e}}$ at low values of $K_{\text {trans }}$, previously detailed in Section 4.7.2.

\subsection{Hemisphere Level Analysis}

While perfusion parameter maps such as those shown in Figure 6.1 show a clear change both over time and between hemispheres, the goal of the analysis in this Section was to obtain a broad but quantitative overview of the temporal and inter-hemisphere variations in each parameter. As such, results in this Section are presented as a single value per hemisphere with an 


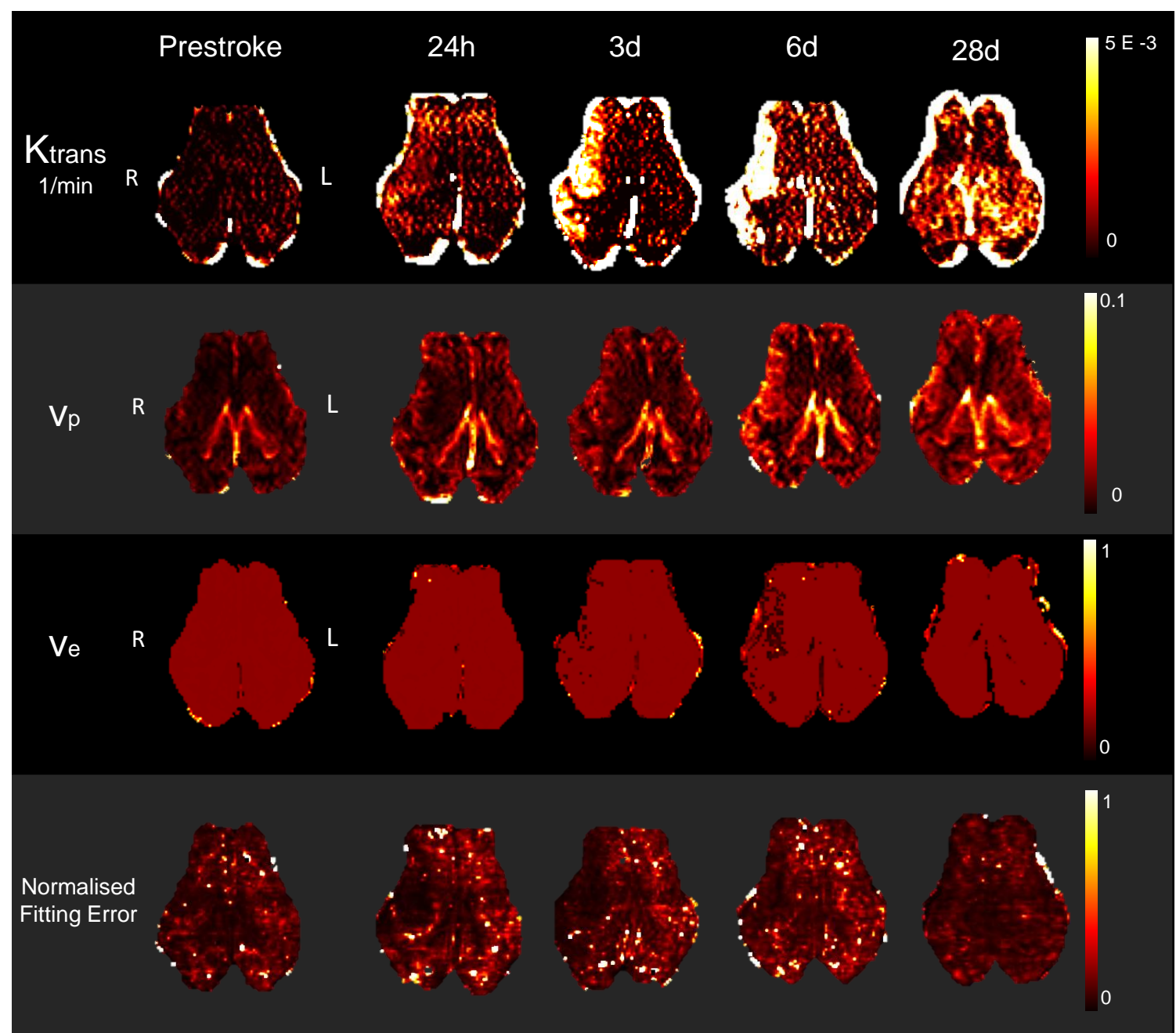

Figure 6.1: Extended Tofts parameter maps for animal RT090. (Top row) $K_{\text {trans }}$, (second row) plasma volume fraction, (third row) interstitial volume fraction and (bottom row) residual of fit normalised to concentration are shown at prestroke and each post-stroke acquisition day. 


\begin{tabular}{|c|c|c|c|c|c|}
\hline Hemisphere & Prestroke & $24 \mathrm{~h}$ & $3 \mathrm{~d}$ & $6 \mathrm{~d}$ & $28 \mathrm{~d}$ \\
\hline Infarcted/Right & 1.00 & 0.56 & 0.69 & 1.19 & 1.77 \\
& $(0.11,0.5,3.92)$ & $(0.09,0.36,1.33)$ & $(0.13,0.40,2.16)$ & $(0.32,0.78,2.39)$ & $(0.08,0.38,8.78)$ \\
\hline Healthy/Left & 1.36 & 0.60 & 0.58 & 0.86 & 2.31 \\
& $(0.12,0.63,4.99)$ & $(0.08,0.38,1.76)$ & $(0.07,0.33,1.12)$ & $(0.25,0.46,2.12)$ & $(0.12,0.36,10.96)$ \\
\hline
\end{tabular}

Table 6.1: Mean infarct and mirror hemisphere values of $K_{\text {trans }}(5$ th percentile, median, 95th percentile) in $\mathrm{min}^{-1} \times 10^{-2}$ at prestroke $(\mathrm{n}=19), 24$ hours $(\mathrm{n}=19), 3$ days $(\mathrm{n}=19), 6$ days $(\mathrm{n}=20)$ and 28 days $(\mathrm{n}=16)$.

indication of spread of values given as the 5th percentile, median, and 95th percentile. These results are summarised in Table 6.1

There are two issues with the data presented in Table 6.1. Firstly, from this Table the values for the comparatively healthy, left hemisphere are higher at 24 hours and 28 days than that of the stroke hemisphere, which is opposite to expected trends from literature [4]. Secondly, the 95th percentile value for several of the acquisition days indicates a very wide spread of values, within which an inter-hemisphere, temporal change can not be discerned at this level of analysis.

These results motivated further analysis of possible confounding factors, the first and most apparent of which was AIF selection.

\subsubsection{AIF Based Exclusion}

The results presented in Table 6.1 were obtained using subject and acquisition day specific AIFs found using the procedure described in Section 5.1.2 with no exclusions. In the present Section, parameter results were included only if the corresponding AIF was visually acceptable. Examples of visually acceptable AIFs are shown in Figure 6.2(a) whereas examples of the AIFs determined to be unsuitable are displayed in Figure 6.2(b).

In the case that the algorithm did not find a visually suitable AIF within the masked anatomy, the fitted parameter values were excluded from the results presented in this Section. 


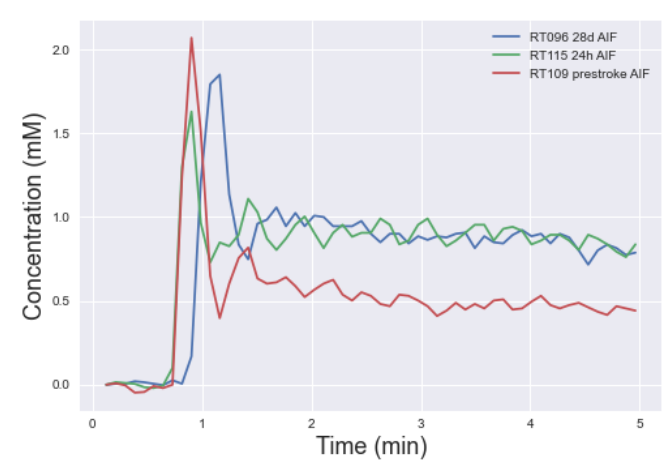

(a)

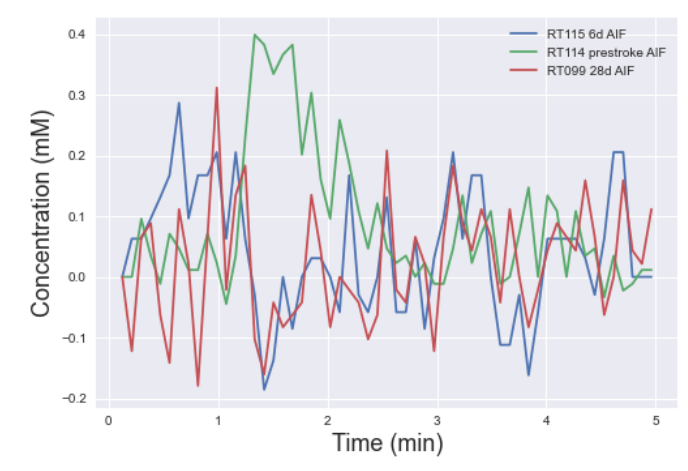

(b)

Figure 6.2: Examples of AIFs deemed to be (a) visually acceptable and (b) visually unsuitable

For those animals where a visually acceptable AIF was found following the above procedure, mean parameter values for the right (stroke) and left (healthy) hemisphere were calculated and are shown in Figure 6.3

From Figure 6.3 we see a difference between the stroke and healthy, contralateral hemisphere on each acquisition day, including on the prestroke day on which we would expect no significant inter-hemisphere difference. The differences seen prior to stroke provide a useful baseline for the level of change that is not due to the stroke, and as such any inter-hemisphere changes of a magnitude lower than the differences we see between the hemispheres at the prestroke timepoint may be rejected as insignificant at this level of analysis.

The 3 day post-stroke values show a small increase in mean $K_{\text {trans }}$ in both the stroke and contralateral hemisphere. The 6 day time point shows the greatest difference between hemispheres, with the mean 6 day $K_{\text {trans }}$ value more than $60 \%$ higher than the equivalent 6 day mirror hemisphere $K_{\text {trans }}$ values; $9.9(3.5,8.3,22) \times 10^{-3} \mathrm{~min}^{-1}$ and $6.1(2.6,4.9,17.1) \times 10^{-3} \mathrm{~min}^{-1}$ respectively.

At 28 days, both hemisphere $K_{\text {trans }}$ values return to levels comparable to the 24 hour and prestroke values. 


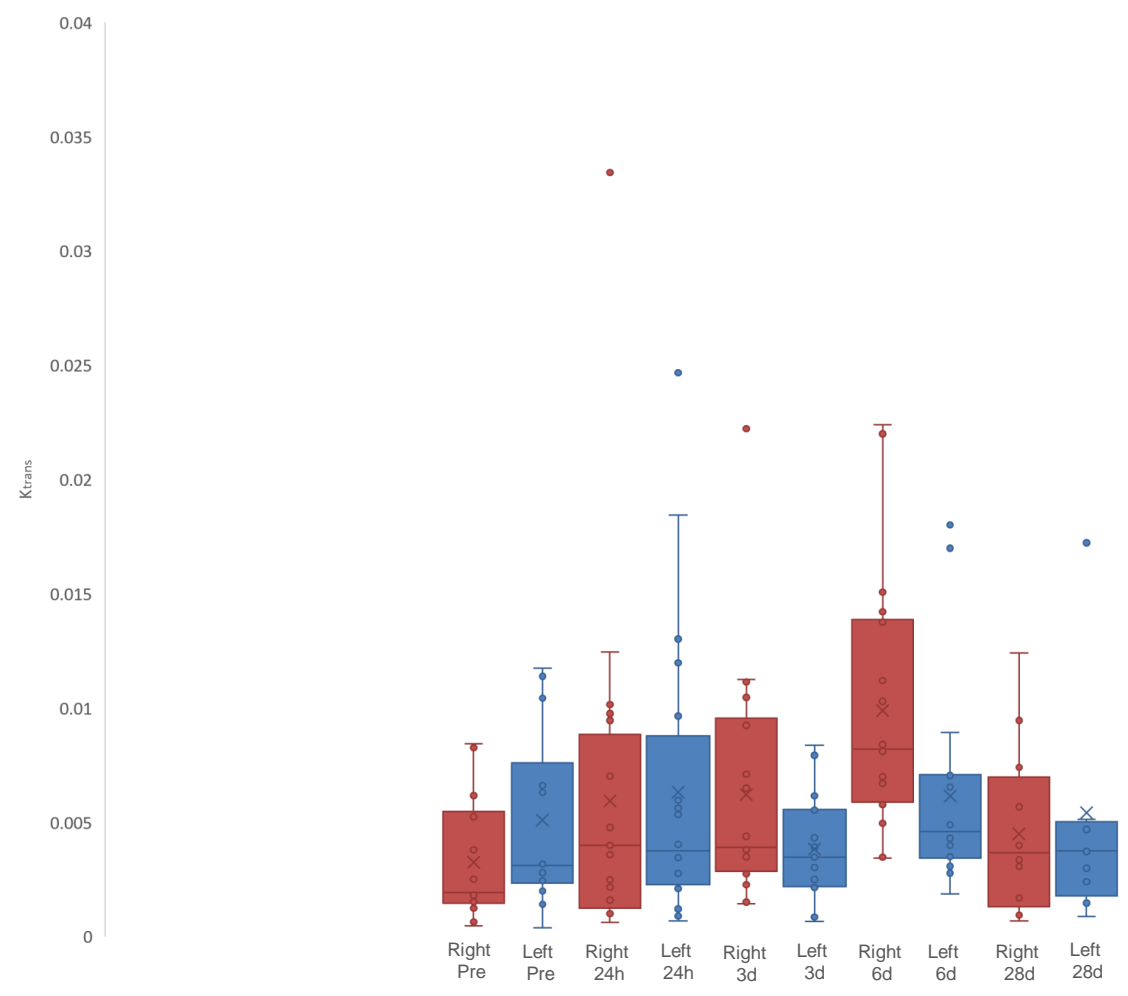

Figure 6.3: Mean $K_{\text {trans }}$ values in right (stroke) and left (opposite) hemisphere on each acquisition day; prestroke $(n=14,6$ animals excluded due to no suitable AIF, 1 animal excluded due to poor quality concentration map), 24 hours post-stroke $(n=20,1$ animal excluded due to no suitable AIF), 3 days post-stroke ( $\mathrm{n}=18,1$ animal excluded due to no suitable AIF, 2 animals had no data for this day), 6 days post-stroke ( $\mathrm{n}=18,2$ animals excluded due to no suitable AIF, 1 animal had no data for this day) and 28 days post-stroke $(\mathrm{n}=12,6$ animals excluded due to no suitable AIF, 3 animals had no data for this day) 
While the trend in $K_{\text {trans }}$ values is more apparent following exclusion of the values corresponding to poor AIFs, the inter-hemisphere difference at the prestroke time point, the presence of many outliers in Figure 6.3, and the high 95th percentile values reported above indicated that isolating the parameter changes within the tissue of interest would require further analysis.

This whole hemisphere level of analysis was useful to determine what would be required in order to obtain more precise parameter estimates and to isolate the range of values over which we see a clear temporal change, if any. The next analysis steps therefore involved:

1. establishing appropriate threshold values for exclusion of outliers and poorly fit voxels from the analysis, and

2. analysis of the $K_{\text {trans }}$ histogram to determine the range over which a significant temporal change may be detected.

These steps are detailed in the following Sections.

\subsubsection{Error in Fit Based Exclusion}

Subsequent analysis at the hemisphere level involved investigating ways to exclude poorly fit voxels from the results. Several physiological phenomena contributed to poorly fit voxels, such as temporal dilation and contraction of blood vessels, signal from ventricles, which are cerebrospinal fluid filled spaces within the brain that did not contribute to meaningful signal, and the inclusion of outliers in the anatomical mask. It was assumed that the signal from the ventricles and other areas where there was no leakage of contrast agent would have a poor fit to the extended Tofts model, as the signal from these areas would not be correlated to the passage of the contrast bolus. As such, exclusion of voxels with a poor fit would potentially remove factors masking the subtle perfusion changes as a result of the transient stroke. Several measures of the goodness of fit between the tissue concentration curve and extended Tofts fit were investigated, including the sum of squared errors, 


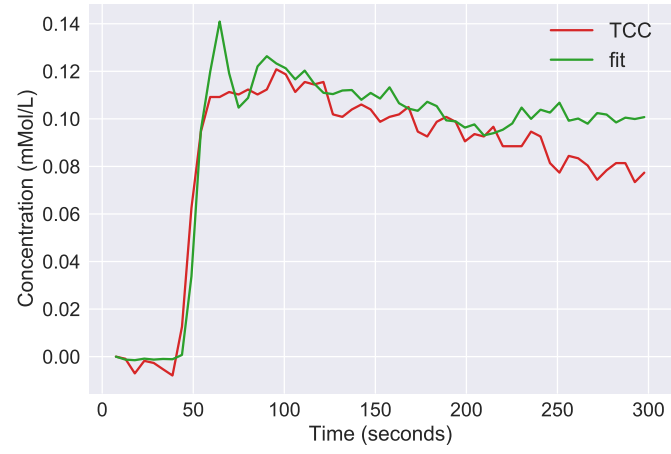

(a)

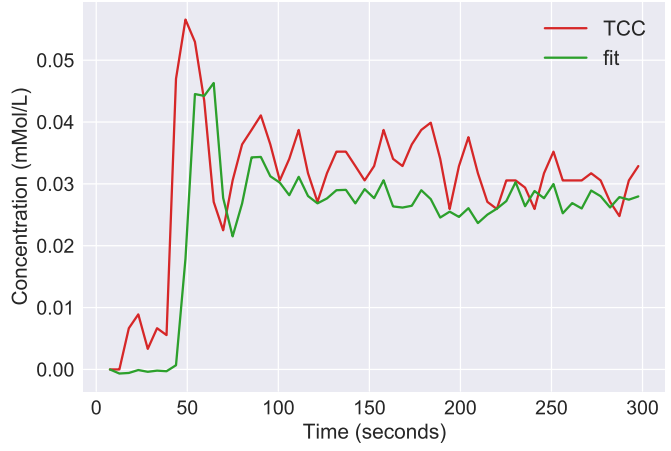

(b)

Figure 6.4: Two tissue concentration curves (red) and extended Tofts parameter fit (green) where (a) $\mathrm{SSE}=0.006, \mathrm{R}^{2}=0.9$ and (b) SSE $=0.004, \mathrm{R}^{2}=0.5$.

a normalised measure of the sum of squared errors, and the coefficient of variation $\left(R^{2}\right)$.

\section{Sum of Squared Errors}

The sum of squared errors was the optimization criteria for fitting of each tissue concentration curve, as described in Section 4.6. However, this measure was not ideal for voxel exclusion due to the relationship between concentration value and sum of squared errors.

This is illustrated with the two example fits given in Figure 6.4. Here, the fit shown in (b) has a lower sum of squared errors than (a), which taking the sum of squared errors as the measure of goodness of fit, would imply that the right plot shows a better fit than the left. However, this is due to the low concentration values of the tissue concentration curve on the right. The plot on the left has a higher sum of squared errors, due to the concentration values being higher in this voxel over time.

To negate this effect, another measure of goodness of fit was investigated. 
$\mathbf{R}^{2}$

The coefficient of variation, $\mathrm{R}^{2}$, provided a measure of how the tissue concentration curve (TCC) and the extended Tofts model fit varied over time, and is given by

$$
\left.R^{2}=1-\frac{\Sigma_{i}\left(C_{\mathrm{t}, i}-f_{i}\right)^{2}}{\Sigma_{i}\left(C_{\mathrm{t}, i}-\overline{C_{\mathrm{t}, i}}\right.}\right)
$$

where $C_{\mathrm{t}, i}$ denotes the tissue concentration curve value at time point $i$, and $f_{i}$ denotes a general fit, in this project, the fit to the extended Tofts model with parameters $K_{\text {trans }}, v_{\mathrm{e}}$ and $v_{\mathrm{p}}$, at time point $i$.

As shown in Figure 6.4, the $\mathrm{R}^{2}$ does not vary with the peak concentration as the sum of squared errors does, making it an attractive choice for exclusion criteria that does not discriminate against higher concentration values.

While in this Section $\mathrm{R}^{2}$ is being presented as an exclusion criteria, in the fitting process applied to the data the optimisation metric was the sum of squared errors. Minimising the sum of squared errors in this case was equivalent to maximising the $\mathrm{R}^{2}$, as shown in equation 6.1. By minimising the numerator of this equation, which is in fact the sum of squared errors, and given that the denominator depends only on the tissue concentration curve and not the extended Tofts fit, $f(t)$, we maximise $R^{2}$.

An interesting result of using $\mathrm{R}^{2}$ as a measurement of the error in fit was in the case of voxels with very low or negative signal enhancement where the best fit found was a straight horizontal line at zero, the $\mathrm{R}^{2}$ returned was NaN (meaning 'not a number'). This was due to the fact that the constant horizontal line of the fit was not varying in time with the tissue concentration curve. By isolating all voxels where $\mathrm{R}^{2}$ returned $\mathrm{NaN}$, the voxels with negative or very low signal enhancement could be detected and excluded from analysis. The anatomical origins of these voxels is discussed further in Section 6.2.3, but at this stage of the analysis these voxels were simply excluded via generation of an $\mathrm{R}^{2}$ map for each animal at each acquisition day and the setting of a threshold $\mathrm{R}^{2}$ value below which a voxel would be excluded. 


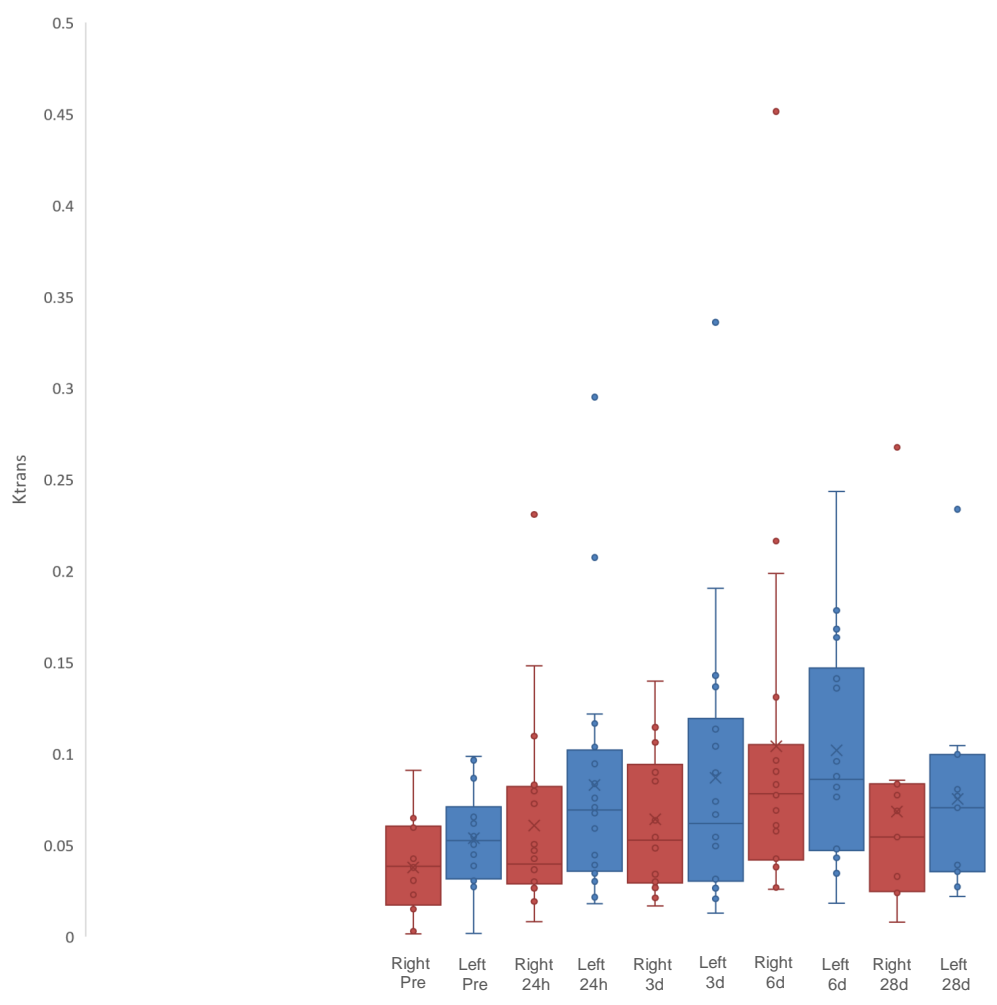

Figure 6.5: Recalculation of data shown in Figure 6.3 with a minimum $\mathrm{R}^{2}$ threshold of 0.8 .

The results presented in Figure 6.3 were recalculated and any voxels below the threshold of $R^{2}=0.8$ were rejected from analysis. These $R^{2}$ based voxel exclusion results are given in Figure 6.5.

Contrary to what was expected, isolation of only the best fit voxels resulted in a vastly different range of parameter values. As seen on the y-axis of Figure 6.5 , the values from the best fit voxels are in the range of $K_{\text {trans }}=0.05 \mathrm{~min}^{-1}$ as opposed to the previous range centred roughly at $K_{\text {trans }}=0.005 \mathrm{~min}^{-1}$ as shown in Figure 6.3.

This result indicated both that the best fit voxels were those with higher $K_{\text {trans }}$ values than the mean values reported previously, and that the broad trend between hemispheres and over time in Figure 6.3 was not present in 
these voxels.

This order of magnitude difference between mean values indicated that there might be a subtlety to the distribution of the parameters which a single mean value could not accurately reflect. Therefore the next analysis step involved investigation of the parameter histogram to determine the spread of values and range over which a significant temporal change might be detected.

\subsubsection{Histogram Level Analysis}

Analysis at the histogram level was conducted to determine the physiologically relevant range of values, i.e. the range of values over which a meaningful change could be measured between post-stroke time points.

The histogram for a typical prestroke animal (RT090 from Table 8.3) is given in Figure 6.6. An example cumulative distribution function is also included for visualisation purposes in Appendix 8.4.

Figure 6.6 shows a natural division in the histogram at $K_{\text {trans }} \approx 0.015 \mathrm{~min}^{-1}$ and the mean values for both the infarct and opposite hemispheres at 0.014 and $0.033 \mathrm{~min}^{-1}$ respectively. This mean value is shown in Figure 6.3, but from the histogram we see that this value is heavily influenced by a small proportion of very high values.

The shape of the histogram in Figure 6.6 indicates that in simply taking the mean value over the entire hemisphere, we are losing information on the more subtle changes within the distribution. A method to account for these two visually distinct segments was splitting the histogram into two segments, 1 and 2 , at $K_{\text {trans }}=0.01 \mathrm{~min}^{-1}$. These segments are visualised in red and blue respectively in Figure 6.7(a).

Following separation of the histogram into two segments, in order to isolate the physiologically relevant changes from the surrounding values, investigation of the anatomical structures relating to each of these segments was undertaken. 


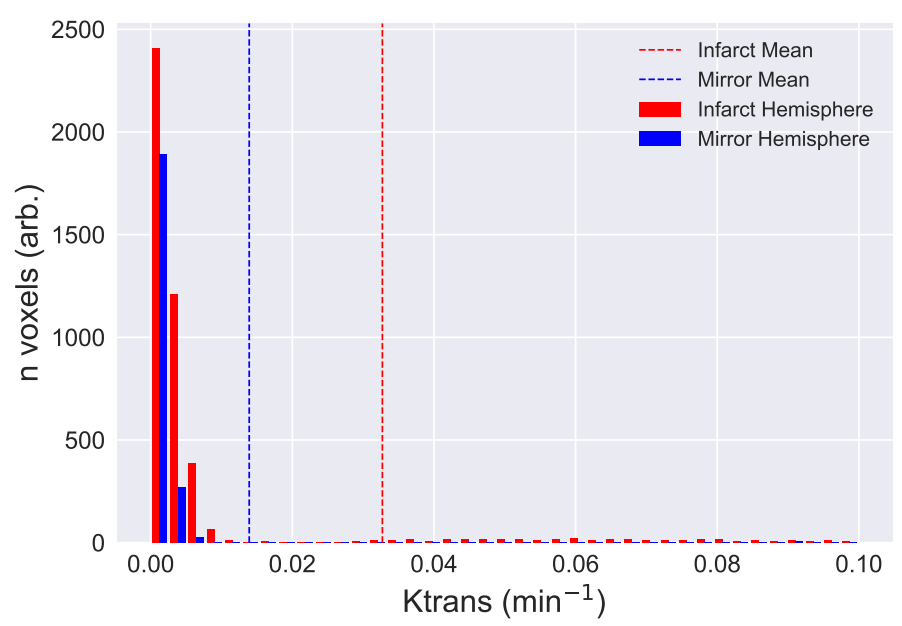

Figure 6.6: Histogram for a 3 day post-stroke animal (RT090). The $x$ axis has been truncated at $K_{\text {trans }}=0.1 \mathrm{~min}^{-1}$ for visualisation purposes.

\section{Consideration of Anatomical Structures}

Up until now, the two histogram segments under consideration have been distinguished only by their location in the histogram distribution, with no investigation into the anatomical origins of these two segments. Analysis of the anatomical origins of these segments is presented here.

Figure 6.7(a) provides an anatomical representation of the proposed split histogram segments. A clear distinction can be made between the cerebral cortex encompassed by segment 1, and the regions in blue.

In Figure 6.7(b) we see a third histogram segment in yellow, from a post-stroke animal, which represents a central segment between the original segment 1 and 2 , specified as $0.01<K_{\text {trans }} \leq 0.1$. This region represents the ischemic tissue, the area of greatest relevance to perfusion measurements, however in the two segment analysis model, all of these voxels were included in segment 2, along with the vasculature and outliers on the edge of the hemisphere mask. Here we have reached a limitation of the two segment 


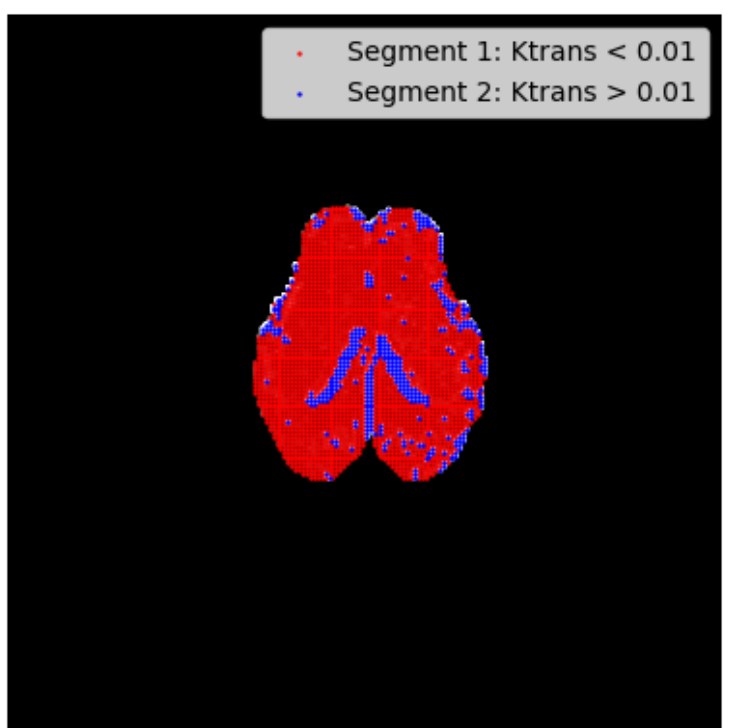

(a)

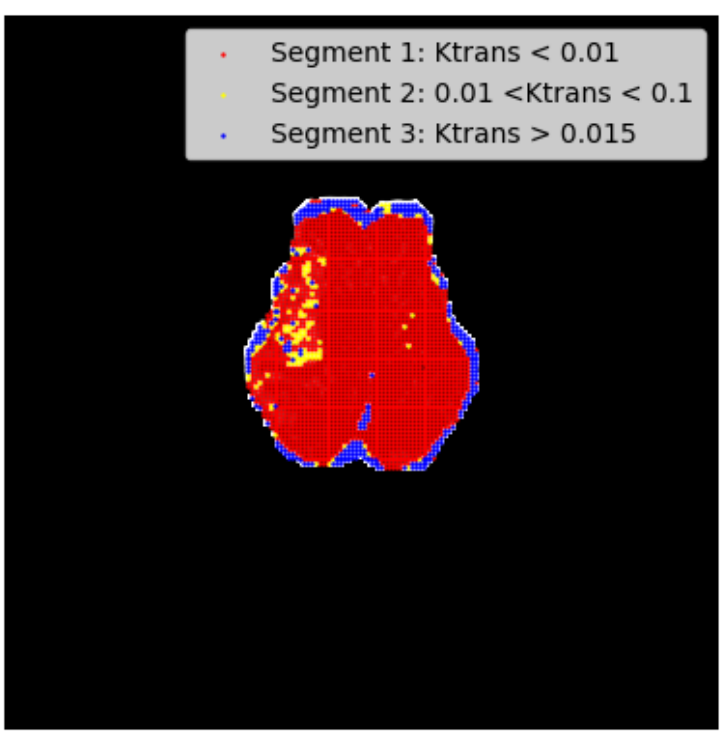

(b)

Figure 6.7: Visualisation of (a) segment 1 and 2 of histogram of animal RT090 prestroke (b) three segment visualisation from poststroke animal isolating infarct voxels. 
histogram analysis technique's capability. Introducing a third segment to this histogram level analysis could further isolate the changes in these relevant voxels, however this third segment would also include vasculature and may exclude the infarct penumbra, which would remain in segment 1.

\subsubsection{Summary of Hemisphere Level Analysis}

This concludes the analysis conducted at the hemisphere level. This broad level of analysis was useful for establishing hemisphere wide perfusion trends, and allowed for quantification of perfusion without further knowledge of the sub-regions within the ischemic tissue. However, at this level of analysis, any heterogeneity in the ischemic tissue is being masked by taking an average parameter value for the entire hemisphere. While various exclusion methods were attempted to remove the confounding influence of ventricle signal from the effects of the stroke, the clearest trend between stroke and contralateral hemisphere can be seen in Figure 6.3. At 6 days post-stroke we see a clear increase, $(\geq 60 \%)$, in stroke hemisphere $K_{\text {trans }}$ values.

While some global trends can be discerned visually in the perfusion parameter maps and Figure 6.3, analysis of the hemisphere histograms indicated that the subtle changes we wish to quantify may not be being accurately represented through this broad level of analysis. As such, further analysis presented in this Chapter is at the small region of interest (ROI) level, and aims to account for the heterogeneity of the ischemic tissue. 


\subsection{Small ROI Level Analysis}

Regions of interest used in this Section are significantly smaller than the whole hemisphere masks used in Section 6.2. While the use of these large masks allowed for investigation of broad trends within the hemisphere, the averaging applied to the volume meant that any subtle perfusion changes between time points were masked by ventricle and vasculature signal, and that any information on heterogeneous tissue regions within a hemisphere was lost.

The goal of analysis in this Section is to account for heterogeneous perfusion changes within the affected hemisphere. As such, it is important at this level of analysis that the ventricles and other areas of irrelevant signal are fully neglected in order to avoid the masking of the physiologically relevant areas. The simplest way of neglecting these voxels was exclusion through selection of small regions of interest placed in physiologically relevant areas derived from diffusion weighted images. Physiological motivation behind the analysis presented in this Section is given in Section 6.3.1, and subsequent sections present more detailed results with consideration of different stroke regions as described in Section 2.1.3

\subsubsection{Consideration of ischemic tissue heterogeneity}

At the hemisphere level of analysis we considered each hemisphere as one region of interest within the brain. However, inspection of the diffusion weighted images reveals that the ischemic tissue in the affected hemisphere is heterogeneous in nature, implying that there are different regions of the ischemic tissue undergoing different physiological processes. As such, averaging over an entire hemisphere may lead to the cancelling out of these physiological effects.

Figure 6.8 shows the distinct regions within the ischemic tissue, as well as clearly showing the ventricles, cerebrospinal fluid filled cavities in the brain that do not demonstrate any meaningful signal enhancement. The ischemic 


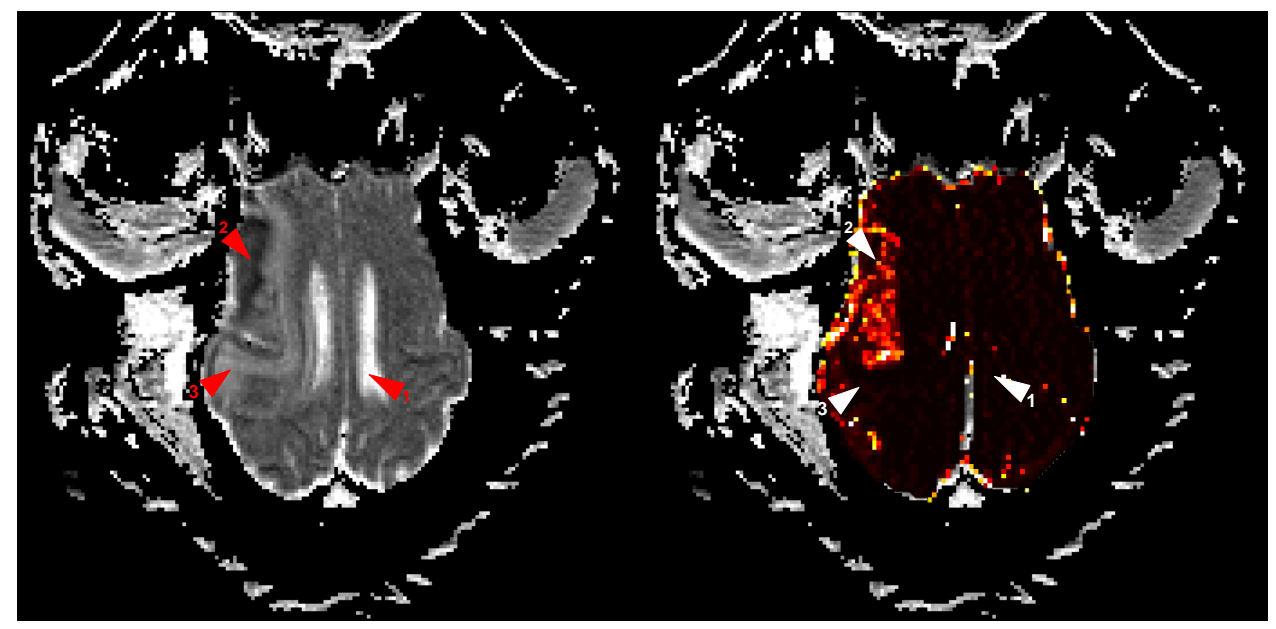

(a)

(b)

Figure 6.8: (a) Diffusion weighted image and (b) corresponding $K_{\text {trans }}$ map of animal RT090 at 6 days post-stroke with physiologically relevant areas labelled with arrows: (1) ventricle (2) cytotoxic edema forming infarct core and (3) vasogenic edema and infarct penumbra.

tissue is made up of two types of edema; cytotoxic edema and vasogenic edema. The cytotoxic edema, labelled with arrow 2 in Figure 6.8 makes up the area of the affected hemisphere in which intracellular swelling and cell membrane pumps have failed. As a result, this region appears dark in the DWI images [5]. The vasogenic edema is the area in which the vessel wall, i.e. the blood brain barrier, has broken down and as such fluid has filled this region and diffusion is less restricted. This area therefore appears bright in DWI images. When comparing the $K_{\text {trans }}$ map to the DWI images, we see the area corresponding to both types of edema is in fact heterogeneous also. In order to quantify the perfusion changes in the different areas of ischemic tissue, small regions of interest were required.

Of note is the relevant clinical time points of both of these types of edema. While the cytotoxic edema occurs from several minutes post-stroke and can endure up to 24 hours, the vasogenic edema forms over time as the blood 


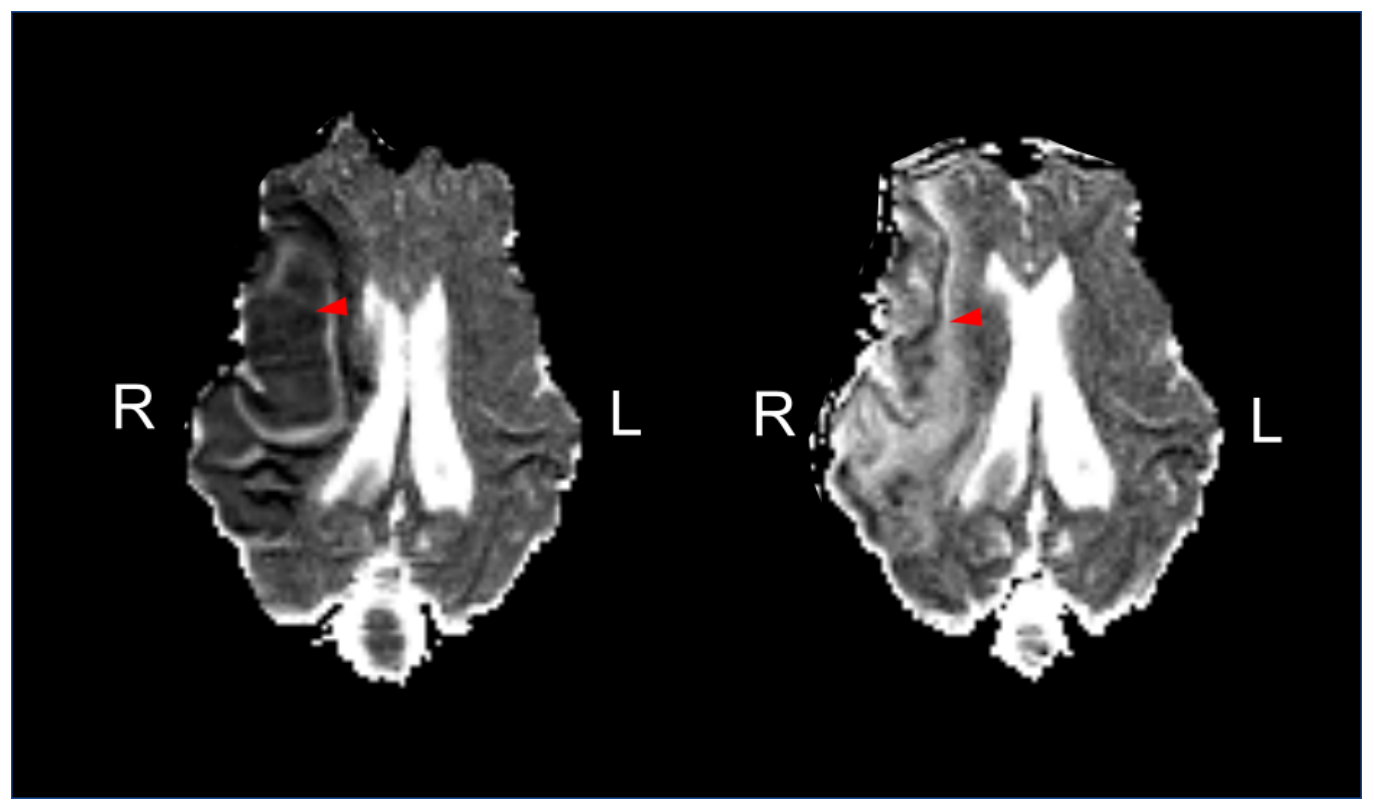

Figure 6.9: Ischemic tissue in the affected hemisphere of animal RT090 at $24 \mathrm{~h}$ (left) demonstrating predominantly cytotoxic edema and at $6 \mathrm{~d}$ (right) demonstrating predominantly vasogenic edema. 
brain barrier breaks down, with the peak vasogenic edema volume expected between 5 and 6 days post-stroke [5]. As such, the 24 hour post-stroke images were used for estimates of cytotoxic perfusion parameter values, and the 3 and 6 day images were used for estimates of vasogenic perfusion parameter values.

\subsubsection{Small ROI Generation}

The goal of this analysis was to quantify the perfusion in cytotoxic and vasogenic edema, and in healthy contralateral tissue, and see if these could be reliably distinguished from perfusion measurements.

These different tissue areas were identified using diffusion weighted images (DWI). The DWI and perfusion images were co-registered and regions of interest were manually placed in each tissue type using the programme 3DSlicer [64]. These regions of interest were then exported with the same geometry as the perfusion maps, and applied to the maps of each parameter.

Each small region of interest was 31.5 cubic millimetres, and six of these ROIs were placed in cytotoxic and healthy tissue in 24 hour images, and vasogenic and healthy tissue in the 6 day images, the average values of which are given in Figure 6.11. As only certain animals had discernible regions of cytotoxic and vasogenic edema, the $\mathrm{n}$ values of this analysis are considerably lower than in the whole hemisphere level analysis.

\subsubsection{Vasogenic, cytotoxic and healthy tissue results.}

Results of small ROI analysis of cytotoxic, vasogenic and healthy tissue showed an increase in $K_{\text {trans }}$ in areas of vasogenic edema compared to healthy tissue and cytotoxic edema. Cytotoxic edema shows $K_{\text {trans }}$ values comparable to those in the healthy tissue at 24 hours post-stroke, but elevated compared to $K_{\text {trans }}$ in healthy tissue at 6 days post-stroke.

The peak we see in hemisphere level $K_{\text {trans }}$ in Figure 6.3 , which is visually exemplified by animal RT090 in Figure 6.1, corresponds to the formation 


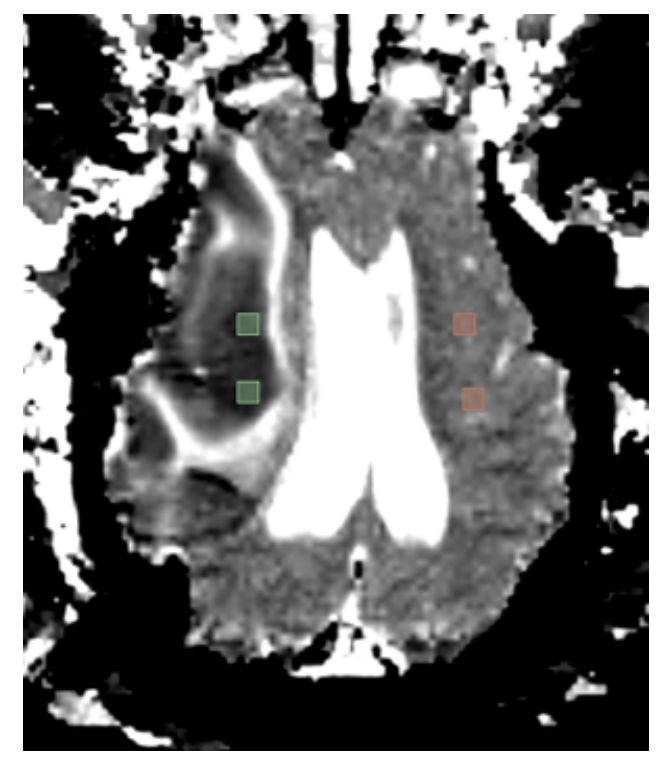

(a)

Figure 6.10: Example of small ROIs placed in region of cytotoxic edema and healthy contralateral tissue in animal RT109 at 24 hours post-stroke. 


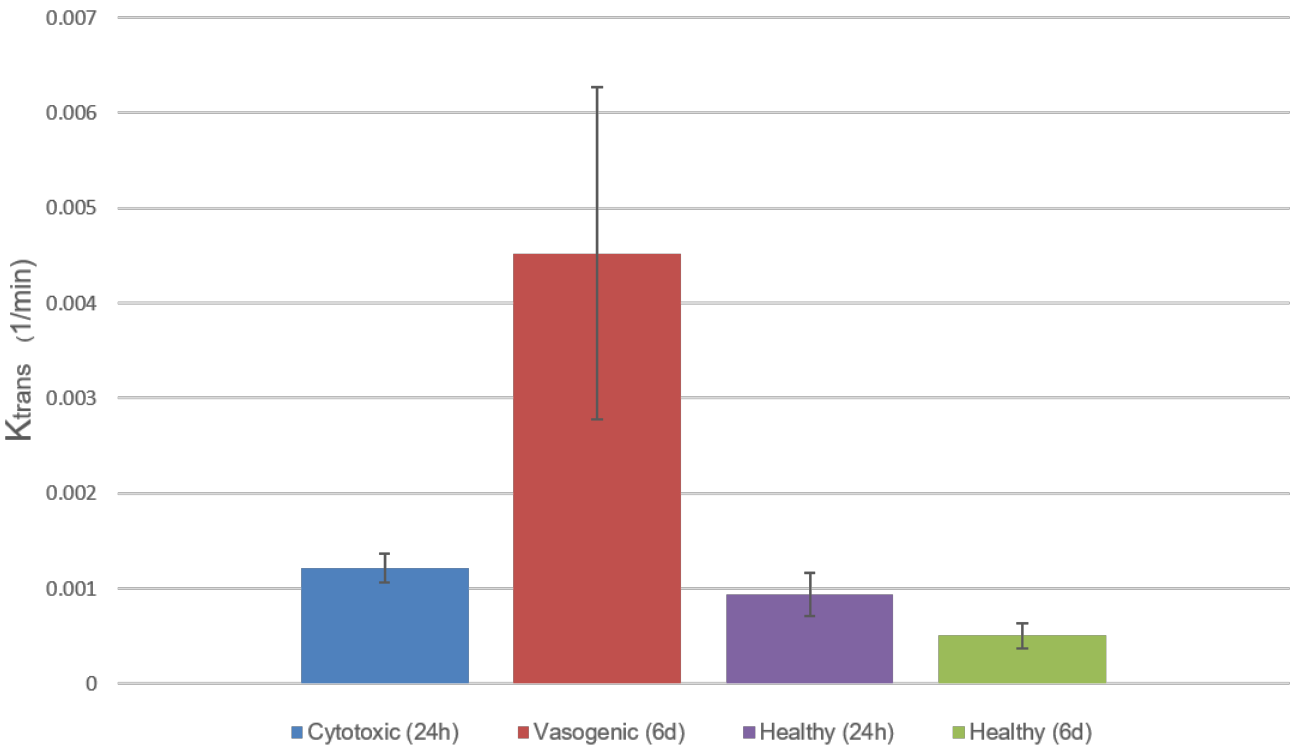

Figure 6.11: Average $K_{\text {trans }}$ values of cytotoxic edema at 24 hours $(n=7)$, vasogenic edema at 6 days $(n=8)$ and contralateral tissue at 24 hours and 6 days ( $n=7$ and 8 respectively) 
of vasogenic edema which is known to peak at 6 days post-stroke. By demonstrating that this novel model of MCA occlusion is able to reproduce the complex perfusion changes we see in humans post-stroke, we have shown this animal model is a good candidate for research into stroke treatments, as it is desirable for the animal model to respond to both ischemic stroke and treatment in a way that is as close to human patterns of disease as possible. As such, the findings presented in this Section lend significant support to the use of this animal model in future research.

Further details on possible avenues for future research, and a summary of the findings from this project are given in Chapter 7 . 


\section{Chapter 7}

\section{Conclusion and Future Research}

The analysis presented within this thesis is the first time the DCE-MRI datasets from this novel animal model of stroke have been analysed. As such, this project required critical thinking at each stage to determine how best to proceed, in terms of experimental methods, image processing techniques and analysis methods. While different options at each step were considered carefully, as is reported in this thesis, with no time constraints, and unbridled access to reference data, several methods used in this project could be improved.

The results reported in this project were concluded with estimates for $K_{\text {trans }}$ within vasogenic and cytotoxic edema at 24 hours and 6 day post-stroke time points, but there remains a wealth of data and options for going forward with analysis, with possible avenues for future research outlined in this section.

\subsection{Conclusions}

Through the experimental methods described in this thesis, the perfusion changes within the brains of these animals were quantified. Hemisphere level changes were detected through various analysis techniques, and more refined 
analysis techniques combining knowledge of the ADC time course in stroke [5] and physiology of the stroke disease mechanism revealed further distinctions in perfusion parameters within different types of edema.

It was found that the vasogenic $K_{\text {trans }}$ values were significantly higher than cytotoxic $K_{\text {trans }}$ values, reflecting the barrier permeability changes known to occur in these types of edema post-stroke. Crucially, the broader increase in $K_{\text {trans }}$ within the affected hemisphere also agrees with known changes in $K_{\text {trans }}$ in humans [4], providing support to the use of this animal model in future research into stroke therapies.

Key findings from this project were:

1. Perfusion from the DCE-MRI series obtained from the animal experiments under consideration in this project can be quantified.

2. There are many factors that can significantly impact the parameter values. In particular, PK model selection, curve fitting techniques and AIF selection.

3. The perfusion values calculated from this animal model reflect known human patterns of disease in terms of $K_{\text {trans }}$ changes in the affected hemisphere, and in regions of cytotoxic and vasogenic edema.

4. At each step there were often many different ways to proceed, but the best approaches were those that united knowledge from both physiology and physics.

Throughout the research reported in this thesis, insights from two areas of knowledge constantly informed decision making. Broadly, these were physics and physiology. For example, in AIF selection, knowledge of sheep anatomy and which artery would serve best as a global AIF was used alongside an automated search for the curve shape characteristic of an AIF. Or in determination of areas of cytotoxic and vasogenic edema, MRI physics in terms of the known ADC time course of ischemic stroke was invaluable in discerning these two types of edema. A key finding from this project is 
therefore that combining knowledge from MRI physics and from physiology results in the most sensible experimental methods, as opposed to considering each problem only a physiology problem, or a signal processing problem.

\subsection{Future Research}

There is a wealth of physiological and MRI data available for this animal cohort. Areas of future research specific to the results presented in this thesis are described in this Section.

\section{Acquisition Improvements}

In order to extract perfusion parameters such as separate estimation of $F_{\mathrm{p}}$ and PS, acquisition of DCE-MRI series with higher temporal resolution, perhaps on the order of 1.5-2 seconds would be ideal. This may, however, compromise spatial resolution, and so alternative methods should be explored for reducing the temporal resolution.

Throughout this project it was invaluable having a point of comparison for calculated values such as $T_{1}, R_{1}$ and synthetic DCE-MRI data. Having a similar point of comparison for the concentration maps would be very useful for verifying the range of expected concentration values following the injection of a known dose of CA.

\subsubsection{Animal characteristic based analysis}

Data relating to the sex of the animals, the infarct size of the animals at each time point, and whether or not the animal received an experimental stroke therapy is available for this cohort of animals. Comparing the perfusion changes between animals who received a therapeutic agent against those that did not, and analysis based on the volume of the ischemic tissue in each animal may yield more interesting results from this data. 


\section{Perfusion-Diffusion mismatch}

These results would serve as a useful starting point for research comparing perfusion-diffusion mismatch in this animal model, given that DWI data for all of these animals is available as part of the comprehensive MRI protocol obtained. Clinically, perfusion-diffusion mismatch is an important emerging method of determining the portion of ischemic tissue in the affected hemisphere that may be salvageable through therapeutic agent.

\section{Future perfusion measurement techniques}

The data presented in this thesis represents a perfusion measurement calculated from images. This dataset may serve as a point of comparison for future research utilising non-contrast perfusion measurement techniques such as arterial spin labelling.

\subsubsection{Summary}

The results of this project reflected both a replication of human pattern of disease, in the form of increased barrier permeability in the stroke hemisphere and regions of vasogenic edema, and a replication of the period of time in which we would expect to see such an increase in humans [5][4]. In summary, this thesis supports the statement that this ovine model of MCAO is a good animal model candidate for future research into stroke therapies. 


\section{Chapter 8}

\section{Appendices}

\subsection{Two compartment exchange model impulse response function}

The full mathematical depiction of the impulse response function used for the two compartment exchange model is provided here and taken from [42].

$$
\begin{gathered}
H_{2 \mathrm{CXM}}(t)=B e^{-m_{1} t}+(1-B) e^{-m_{2} t} \\
m_{1}=\frac{1}{2}\left(a+b+\sqrt{(a+b)^{2}-4 b c}\right) \\
m_{2}=\frac{1}{2}\left(a+b-\sqrt{(a+b)^{2}-4 b c}\right) \\
B=\frac{m_{2}-c}{m_{2}-m_{1}} \\
a=\frac{F_{\mathrm{p}}+\mathrm{PS}}{v_{\mathrm{p}}} \\
b=\frac{\mathrm{PS}}{v_{\mathrm{e}}} \\
c=\frac{F_{\mathrm{p}}}{v_{\mathrm{p}}}
\end{gathered}
$$




\subsection{Table of Abbreviations}

\begin{tabular}{|c|c|}
\hline Acronym & Definition \\
\hline $2 \mathrm{CXM}$ & Two Compartment Exchange Model \\
\hline $\mathrm{BBB}$ & Blood Brain Barrier \\
\hline $\mathrm{HPC}$ & High Performance Computing Cluster \\
\hline PS & Permeability surface area product \\
\hline $\mathrm{RS}$ & ROCKETSHIP Matlab software package \\
\hline TCC & Tissue Concentration Curve \\
\hline $\mathrm{ADC}$ & Apparent Diffusion Coefficient (of water molecules) \\
\hline $\mathrm{AIF}$ & Arterial Input Function \\
\hline BAT & Bolus Arrival Time \\
\hline $\mathrm{C}_{p}$ & Plasma concentration (of AIF) \\
\hline $\mathrm{C}_{\mathrm{t}}$ & Tissue concentration \\
\hline $\mathrm{CA}$ & Contrast Agent \\
\hline CSF & Cerebrospinal Fluid \\
\hline DCE-MRI & Dynamic Contrast Enhanced Magnetic Resonance Imaging \\
\hline DWI & Diffusion Weighted Images \\
\hline $\mathrm{F}_{p}$ & Cerebral blood flow \\
\hline Gd & Gadovist \\
\hline HT & Hemorrhagic Transformation \\
\hline $\mathrm{HPC}$ & High Performance Computing (Cluster) \\
\hline $\mathrm{ICP}$ & Intracranial Pressure \\
\hline$K_{\text {trans }}$ & Forward transfer constant \\
\hline $\mathrm{MCA}(\mathrm{O})$ & Middle Cerebral Artery (Occlusion) \\
\hline MRA & Magnetic Resonance Angiography \\
\hline MTT & Mean Transit Time \\
\hline PK & Pharmacokinetic (model) \\
\hline TK & Tracer Kinetics \\
\hline$R_{1}$ & Longitudinal relaxation rate \\
\hline$T_{1}$ & Longitudinal relaxation time \\
\hline TWIST & Time-resolved angiography With Interleaved Stochastic Trajectories \\
\hline$v_{e}$ & Interstitial volume fraction \\
\hline$v_{p}$ & Plasma volume fraction \\
\hline VIBE & Volumetric Interpolated Breath-hold Examination \\
\hline
\end{tabular}




\subsection{Infarct Size Table}

Table of infarct size volumes at 24 hours post-stroke for all animals in this cohort. Infarct sizes quantified using the program ITK-SNAP by students at the University of Nottingham [89].

\begin{tabular}{|c|c|}
\hline Sheep ID & Infarct size (n voxels) \\
\hline RT102 & 13705 \\
RT108 & 9921 \\
RT090 & 8714 \\
RT114 & 7780 \\
RT109 & 7243 \\
RT113 & 6168 \\
RT110 & 3047 \\
RT104 & 2455 \\
RT099 & 1232 \\
RT100 & 1129 \\
RT103 & 1056 \\
RT111 & 760 \\
RT098 & 731 \\
RT107 & 456 \\
RT106 & 436 \\
RT105 & 339 \\
RT101 & 209 \\
RT112 & 172 \\
RT095 & 135 \\
RT097 & - \\
RT115 & - \\
\hline
\end{tabular}




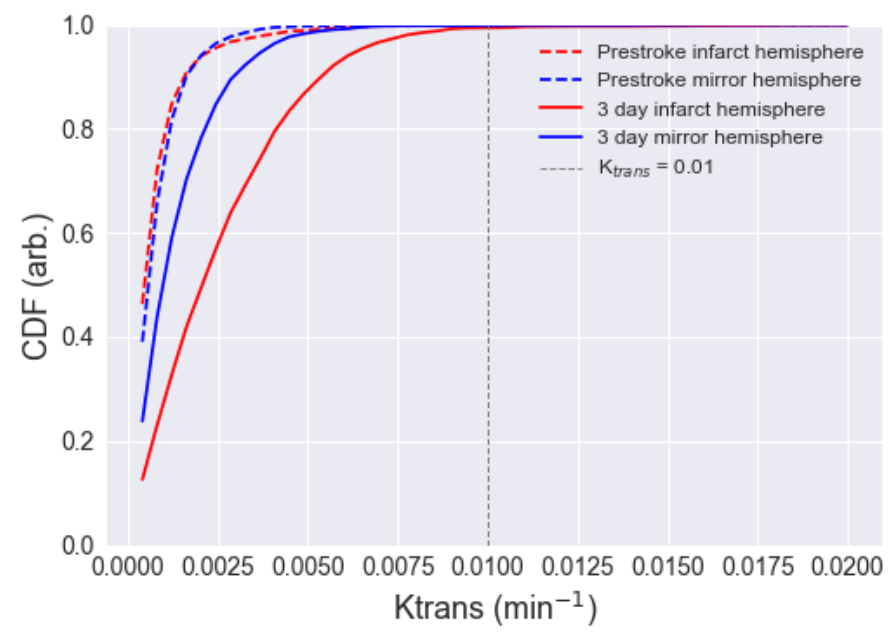

Figure 8.1: Cumulative distribution function for RT090 at 3 days post-stroke.

\subsection{CDF Analysis}

Another analysis technique attempted in this project was generation of cumulative distribution functions. Cumulative distribution functions (CDFs) were calculated for each animal on each acquisition day.

The motivation behind generation of the CDFs was the fact that, similarly to the histogram, all voxels could be represented, but the CDF allowed for better visualisation of regions of change. The y-axis of these functions represents the probability that a voxel value will be below the corresponding parameter value on the x-axis. As such, the maximum possible value is 1 , that is, there is a $100 \%$ probability that a voxel's $K_{\text {trans }}$ value is equal to or below the maximum $K_{\text {trans }}$ value set.

While the example provided in Figure 8.1 shows a clear difference in $K_{\text {trans }}$ value distribution between prestroke and 3 days post-stroke, and between affected and healthy hemispheres, not all animals produced CDFs with the same changes seen here. Analysis was conducted on all CDFs to return the $\mathrm{CDF}_{75}$ value in both hemispheres, however this did not reveal a significant 
difference except for the most affected animals which were already identified. The CDF level analysis therefore served only to demonstrate that more detailed analysis of heterogeneous changes in ischemic tissue was required. 


\section{Bibliography}

[1] E. J. Benjamin, M. J. Blaha, S. E. Chiuve, M. Cushman, S. R. Das, R. Deo, S. D. de Ferranti, J. Floyd, M. Fornage, C. Gillespie, C. R. Isasi, M. C. Jiménez, L. C. Jordan, S. E. Judd, D. Lackland, J. H. Lichtman, L. Lisabeth, S. Liu, C. T. Longenecker, R. H. Mackey, K. Matsushita, D. Mozaffarian, M. E. Mussolino, K. Nasir, R. W. Neumar, L. Palaniappan, D. K. Pandey, R. R. Thiagarajan, M. J. Reeves, M. Ritchey, C. J. Rodriguez, G. A. Roth, W. D. Rosamond, C. Sasson, A. Towfighi, C. W. Tsao, M. B. Turner, S. S. Virani, J. H. Voeks, J. Z. Willey, J. T. Wilkins, J. H. Wu, H. M. Alger, S. S. Wong, P. Muntner, and American Heart Association Statistics Committee and Stroke Statistics Subcommittee, "Heart Disease and Stroke Statistics-2017 Update: A Report From the American Heart Association," eng, Circulation, vol. 135, no. 10, e146e603, Mar. 2017.

[2] N. Ministry of Health, "Mortality 2017 data tables," Mortality and Demographic Data annual series., Dec. 2019.

[3] M. Fisher, G. Feuerstein, D. W. Howells, P. D. Hurn, T. A. Kent, S. I. Savitz, and E. H. Lo, "Update of the Stroke Therapy Academic Industry Roundtable Preclinical Recommendations," en, Stroke, vol. 40, no. 6, pp. 2244-2250, Jun. 2009.

[4] K. Villringer, B. E. S. Cuesta, A.-C. Ostwaldt, U. Grittner, P. Brunecker, A. A. Khalil, K. Schindler, O. Eisenblätter, H. Audebert, and J. B. 
Fiebach, "DCE-MRI blood-brain barrier assessment in acute ischemic stroke," en, Neurology, vol. 88, no. 5, pp. 433-440, Jan. 2017.

[5] Hsia Amie W., Luby Marie, Cullison Kaylie, Burton Shannon, Armonda Rocco, Liu Ai-Hsi, Leigh Richard, Nadareishvili Zurab, Benson Richard T., Lynch John K., and Latour Lawrence L., "Rapid Apparent Diffusion Coefficient Evolution After Early Revascularization," Stroke, vol. 50, no. 8, pp. 2086-2092, Aug. 2019.

[6] S. H. Kim, A. Kamaya, and J. K. Willmann, "CT Perfusion of the Liver: Principles and Applications in Oncology," Radiology, vol. 272, no. 2, pp. 322-344, Jul. 2014.

[7] M. Marenzana and T. R. Arnett, "The Key Role of the Blood Supply to Bone," en, Bone Research, vol. 1, no. 1, pp. 203-215, Dec. 2013.

[8] P. Johns, "Chapter 10 - stroke," in Clinical Neuroscience, P. Johns, Ed., Churchill Livingstone, 2014, pp. 115-128.

[9] D. Purves, G. J. Augustine, D. Fitzpatrick, L. C. Katz, A.-S. LaMantia, J. O. McNamara, and S. M. Williams, "The Blood Supply of the Brain and Spinal Cord," en, in Neuroscience. 2nd edition, 2001.

[10] S. F. N. Zealand and N. Z. I. of Economic Research, The social and economic costs of stroke in new zealand 2020, 2020.

[11] K. M. Barrett and J. F. Meschia, Stroke. Hoboken, UNITED KINGDOM: John Wiley \& Sons, Incorporated, 2013.

[12] P. Duncan, S. Studenski, L. Richards, S. Gollub, S. M. Lai, D. Reker, S. Perera, J. Yates, V. Koch, S. Rigler, and D. Johnson, "Randomized Clinical Trial of Therapeutic Exercise in Subacute Stroke," Stroke, vol. 34, no. 9, pp. 2173-2180, Sep. 2003.

[13] T. Truelsen, S. Begg, and C. Mathers, "The global burden of cerebrovascular disease," en, Cerebrovascular disease, p. 67, 2006. 
[14] M. H. Wilson, "Monro-Kellie 2.0: The dynamic vascular and venous pathophysiological components of intracranial pressure," eng, Journal of Cerebral Blood Flow and Metabolism: Official Journal of the International Society of Cerebral Blood Flow and Metabolism, vol. 36, no. 8, pp. 1338-1350, Aug. 2016.

[15] S. Sharma, M. F. Hashmi, and A. Kumar, "Intracranial Hypertension," eng, in StatPearls, Treasure Island (FL): StatPearls Publishing, 2021.

[16] D. Z. Wang, D. S. Nair, and A. V. Talkad, "Acute Decompressive Hemicraniectomy to Control High Intracranial Pressure in Patients with Malignant MCA Ischemic Strokes," en, Current Treatment Options in Cardiovascular Medicine, vol. 13, no. 3, pp. 225-232, Jun. 2011.

[17] Thorén Magnus, Azevedo Elsa, Dawson Jesse, Egido Jose A., Falcou Anne, Ford Gary A., Holmin Staffan, Mikulik Robert, Ollikainen Jyrki, Wahlgren Nils, and Ahmed Niaz, "Predictors for Cerebral Edema in Acute Ischemic Stroke Treated With Intravenous Thrombolysis," Stroke, vol. 48, no. 9, pp. 2464-2471, Sep. 2017.

[18] Z. Dostovic, E. Dostovic, D. Smajlovic, O. C. Ibrahimagic, and L. Avdic, "Brain Edema After Ischaemic Stroke," Medical Archives, vol. 70, no. 5, pp. 339-341, Oct. 2016.

[19] S. Michinaga and Y. Koyama, "Pathogenesis of Brain Edema and Investigation into Anti-Edema Drugs," International Journal of Molecular Sciences, vol. 16, no. 5, pp. 9949-9975, Apr. 2015.

[20] S. I. Savitz, J.-C. Baron, M. Fisher, N. Sanossian, S. Arbe-Barnes, J. Boltze, J. Broderick, K. O. Broschat, M. S. V. Elkind, D. En'Wezoh, A. J. Furlan, P. B. Gorelick, J. Grotta, A. M. Hancock, D. C. Hess, W. Holt, G. Houser, A. W. Hsia, W.-K. Kim, W. S. Korinek, N. Le Moan, M. Liberman, S. Lilienfeld, M. Luby, J. K. Lynch, C. Mansi, A. N. Simpkins, Z. Nadareishvili, R. G. Nogueira, K. E. Pryor, N. Sanossian, L. H. Schwamm, M. Selim, K. N. Sheth, J. Spilker, Y. Solberg, G. K. Steinberg, S. Stice, M. Tymianski, L. R. Wechsler, and A. J. Yoo, "Stroke 
Treatment Academic Industry Roundtable X: Brain Cytoprotection Therapies in the Reperfusion Era," en, Stroke, vol. 50, no. 4, pp. 1026 1031, Apr. 2019.

[21] V. E. O'Collins, "1,026 Experimental treatments in acute stroke O’Collins - 2006 - Annals of Neurology - Wiley Online Library," 2006.

[22] A. J. Sorby-Adams, R. Vink, and R. J. Turner, "Large animal models of stroke and traumatic brain injury as translational tools," American Journal of Physiology, vol. 315, no. 2, 2018.

[23] M. R. Macleod, T. O'Collins, D. W. Howells, and G. A. Donnan, "Pooling of Animal Experimental Data Reveals Influence of Study Design and Publication Bias \textbar Stroke," Stroke, vol. 35, no. 5, pp. 12031208, 2004.

[24] J. A. Zivin, M. Fisher, U. DeGirolami, C. C. Hemenway, and J. A. Stashak, "Tissue plasminogen activator reduces neurological damage after cerebral embolism," eng, Science (New York, N.Y.), vol. 230, no. 4731, pp. 1289-1292, Dec. 1985.

[25] R. J. Traystman, "Animal Models of Focal and Global Cerebral Ischemia," en, ILAR Journal, vol. 44, no. 2, pp. 85-95, Jan. 2003.

[26] I. M. Macrae, "Preclinical stroke research - advantages and disadvantages of the most common rodent models of focal ischaemia," en, British Journal of Pharmacology, vol. 164, no. 4, pp. 1062-1078, 2011.

[27] A. J. Wells, R. Vink, S. C. Helps, S. J. Knox, P. C. Blumbergs, and R. J. Turner, "Elevated Intracranial Pressure and Cerebral Edema following Permanent MCA Occlusion in an Ovine Model," PLoS ONE, vol. 10, no. 6, Jun. 2015.

[28] A. Durand, F. Chauveau, T.-H. Cho, R. Bolbos, J.-B. Langlois, L. Hermitte, M. Wiart, Y. Berthezène, and N. Nighoghossian, "Spontaneous Reperfusion after In Situ Thromboembolic Stroke in Mice," en, PLOS ONE, vol. 7, no. 11, e50083, Nov. 2012. 
[29] Z. Kotwica, H. G. Hårdemark, and L. Persson, "Intracranial pressure changes following middle cerebral artery occlusion in rats," en, Research in Experimental Medicine, vol. 191, no. 1, pp. 99-104, Dec. 1991.

[30] C. McCabe, M. M. Arroja, E. Reid, and I. M. Macrae, "Animal models of ischaemic stroke and characterisation of the ischaemic penumbra," en, Neuropharmacology, Cerebral Ischemia, vol. 134, pp. 169-177, May 2018 .

[31] D. W. McRobbie, E. A. Moore, M. J. Graves, and M. R. Prince, "Acronyms anonymous ii: Gradient echo," in MRI from Picture to Proton, 3rd ed. Cambridge University Press, 2017, pp. 207-224.

[32] N. Binesh, "Brain Perfusion; How \& Why," en, p. 10, 2012.

[33] F. Lersy, G. Boulouis, O. Clément, H. Desal, R. Anxionnat, J. Berge, C. Boutet, A. Kazémi, N. Pyatigorskaya, A. Lecler, S. Saleme, M. Edjlali-Goujon, B. Kerleroux, D. Ben Salem, S. Kremer, and F. Cotton, "Consensus Guidelines of the French Society of Neuroradiology (SFNR) on the use of Gadolinium-Based Contrast agents (GBCAs) and related MRI protocols in Neuroradiology," en, Journal of Neuroradiology, vol. 47, no. 6, pp. 441-449, Nov. 2020.

[34] N. Wake, H. Chandarana, H. Rusinek, K. Fujimoto, L. Moy, D. K. Sodickson, and S. G. Kim, "Accuracy and precision of quantitative DCE-MRI parameters: How should one estimate contrast concentration?" Magnetic resonance imaging, vol. 52, pp. 16-23, Oct. 2018.

[35] J. Kallehauge, DCE Post-Processing $\mathscr{G}$ Modeling, 2018.

[36] S. Sourbron, M. Ingrisch, A. Siefert, M. Reiser, and K. Herrmann, "Quantification of cerebral blood flow, cerebral blood volume, and blood-brainbarrier leakage with DCE-MRI," en, Magnetic Resonance in Medicine, vol. 62 , no. 1 , pp. 205-217, 2009. 
[37] M. Ingrisch and S. P. Sourbron, "Tracer-kinetic modeling of dynamic contrast-enhanced MRI and CT: A primer," en, Journal of Pharmacokinetics and Pharmacodynamics, vol. 40, no. 3, pp. 281-300, Jun. 2013.

[38] S. P. Sourbron, "Technical aspects of MR perfusion," en, European Journal of Radiology, Diffusion and Perfusion of the Body, vol. 76, no. 3, pp. 304-313, Dec. 2010.

[39] F. Khalifa, A. Soliman, A. El-Baz, M. Abou El-Ghar, T. El-Diasty, G. Gimel'farb, R. Ouseph, and A. C. Dwyer, "Models and methods for analyzing DCE-MRI: A review: Models and methods for analyzing DCE-MRI," en, Medical Physics, vol. 41, no. 12, p. 124301, Nov. 2014.

[40] S. L. Barnes, J. G. Whisenant, M. E. Loveless, and T. E. Yankeelov, "Practical dynamic contrast enhanced MRI in small animal models of cancer: Data acquisition, data analysis, and interpretation," eng, Pharmaceutics, vol. 4, no. 3, pp. 442-478, 2012.

[41] R. H. El Khouli, K. J. Macura, M. A. Jacobs, T. H. Khalil, I. R. Kamel, A. Dwyer, and D. A. Bluemke, "Dynamic Contrast-Enhanced MRI of the Breast: Quantitative Method for Kinetic Curve Type Assessment," en, American Journal of Roentgenology, vol. 193, no. 4, W295-W300, Oct. 2009.

[42] F. Khalifa, A. Soliman, A. El-Baz, M. Abou El-Ghar, T. El-Diasty, G. Gimel'farb, R. Ouseph, and A. C. Dwyer, "Models and methods for analyzing DCE-MRI: A review: Models and methods for analyzing DCE-MRI," en, Medical Physics, vol. 41, no. 12, p. 124301, Nov. 2014.

[43] K. J. Macura, R. Ouwerkerk, M. A. Jacobs, and D. A. Bluemke, "Patterns of Enhancement on Breast MR Images: Interpretation and Imaging Pitfalls," RadioGraphics, vol. 26, no. 6, pp. 1719-1734, Nov. 2006.

[44] T. S. Koh, S. Bisdas, D. M. Koh, and C. H. Thng, "Fundamentals of tracer kinetics for dynamic contrast-enhanced MRI," en, Journal of Magnetic Resonance Imaging, vol. 34, no. 6, pp. 1262-1276, 2011. 
[45] H. H. Billett, "Hemoglobin and Hematocrit," eng, in Clinical Methods: The History, Physical, and Laboratory Examinations, H. K. Walker, W. D. Hall, and J. W. Hurst, Eds., 3rd, Boston: Butterworths, 1990.

[46] S. P. Sourbron and D. L. Buckley, "Tracer kinetic modelling in MRI: Estimating perfusion and capillary permeability," en, Physics in Medicine and Biology, vol. 57, no. 2, R1-R33, Dec. 2011.

[47] P. S. Tofts, "Modeling tracer kinetics in dynamic Gd-DTPA MR imaging," en, Journal of Magnetic Resonance Imaging, vol. 7, no. 1, pp. 91101, 1997.

[48] O. Wu, DSC-MRI: Analysis.

[49] S. P. Sourbron and D. L. Buckley, "Classic models for dynamic contrastenhanced MRI: CLASSIC MODELS FOR DCE-MRI," en, NMR in Biomedicine, vol. 26, no. 8, pp. 1004-1027, Aug. 2013.

[50] H. A. Haroon, D. L. Buckley, T. A. Patankar, G. R. Dow, S. A. Rutherford, D. Balériaux, and A. Jackson, "A comparison of Ktrans measurements obtained with conventional and first pass pharmacokinetic models in human gliomas," en, Journal of Magnetic Resonance Imaging, vol. 19, no. 5, pp. 527-536, 2004.

[51] J. H. Naish, L. E. Kershaw, D. L. Buckley, A. Jackson, J. C. Waterton, and G. J. M. Parker, "Modeling of contrast agent kinetics in the lung using T1-weighted dynamic contrast-enhanced MRI," en, Magnetic Resonance in Medicine, vol. 61, no. 6, pp. 1507-1514, 2009.

[52] X. Tao, L. Wang, Z. Hui, L. Liu, F. Ye, Y. Song, Y. Tang, Y. Men, T. Lambrou, Z. Su, X. Xu, H. Ouyang, and N. Wu, "DCE-MRI Perfusion and Permeability Parameters as predictors of tumor response to CCRT in Patients with locally advanced NSCLC," en, Scientific Reports, vol. 6, no. 1, p. 35 569, Oct. 2016. 
[53] Z. Merali, T. Wong, J. Leung, M. M. Gao, D. Mikulis, and A. Kassner, "Dynamic contrast-enhanced MRI and CT provide comparable measurement of blood-brain barrier permeability in a rodent stroke model," en, Magnetic Resonance Imaging, vol. 33, no. 8, pp. 1007-1012, Oct. 2015.

[54] H. Chen, N. Liu, Y. Li, M. Wintermark, A. Jackson, B. Wu, Z. Su, F. Chen, J. Hu, Y. Zhang, and G. Zhu, "Mismatch of Low Perfusion and High Permeability Predicts Hemorrhagic Transformation Region in Acute Ischemic Stroke Patients Treated with Intra-arterial Thrombolysis," Scientific Reports, vol. 6, Jun. 2016.

[55] S. Bernardo-Castro, J. A. Sousa, A. Brás, C. Cecília, B. Rodrigues, L. Almendra, C. Machado, G. Santo, F. Silva, L. Ferreira, I. Santana, and J. Sargento-Freitas, "Pathophysiology of Blood-Brain Barrier Permeability Throughout the Different Stages of Ischemic Stroke and Its Implication on Hemorrhagic Transformation and Recovery," eng, Frontiers in Neurology, vol. 11, p. 594672, 2020.

[56] R. Veksler, I. Shelef, and A. Friedman, "Blood-Brain Barrier Imaging in Human Neuropathologies," Archives of medical research, vol. 45, no. 8, pp. 646-652, Nov. 2014.

[57] S. Saleem, P. D. Teal, W. B. Kleijn, T. O’Donnell, T. Witter, and Y.-C. Tzeng, "Non-Linear Characterisation of Cerebral Pressure-Flow Dynamics in Humans," PLOS ONE, vol. 10, no. 9, pp. 1-19, 2015.

[58] R. B. Panerai, N. E. Dineen, F. G. Brodie, and T. G. Robinson, "Spontaneous fluctuations in cerebral blood flow regulation: Contribution of PaCO2," Journal of Applied Physiology, vol. 109, no. 6, pp. 1860-1868, Sep. 2010.

[59] A. J. Sorby-Adams, A. V. Leonard, L. E. Elms, O. C. Marian, J. W. Hoving, N. Yassi, R. Vink, E. Thornton, and R. J. Turner, "Determining the Temporal Profile of Intracranial Pressure Changes Following Transient Stroke in an Ovine Model," eng, Frontiers in Neuroscience, vol. 13, p. 587, 2019. 
[60] S. R. Barnes, T. S. C. Ng, N. Santa-Maria, A. Montagne, B. V. Zlokovic, and R. E. Jacobs, "ROCKETSHIP: A flexible and modular software tool for the planning, processing and analysis of dynamic MRI studies," BMC Medical Imaging, vol. 15, Jun. 2015.

[61] D. S. Smith, X. Li, L. R. Arlinghaus, T. E. Yankeelov, and E. B. Welch, "DCEMRI.jl: A fast, validated, open source toolkit for dynamic contrast enhanced MRI analysis," eng, PeerJ, vol. 3, e909, 2015.

[62] C. T. Rueden, J. Schindelin, M. C. Hiner, B. E. DeZonia, A. E. Walter, E. T. Arena, and K. W. Eliceiri, "ImageJ2: ImageJ for the next generation of scientific image data," BMC Bioinformatics, vol. 18, no. 1, p. 529, Nov. 2017.

[63] J. Schindelin, I. Arganda-Carreras, E. Frise, V. Kaynig, M. Longair, T. Pietzsch, S. Preibisch, C. Rueden, S. Saalfeld, B. Schmid, J.-Y. Tinevez, D. J. White, V. Hartenstein, K. Eliceiri, P. Tomancak, and A. Cardona, "Fiji: An open-source platform for biological-image analysis," en, Nature Methods, vol. 9, no. 7, pp. 676-682, Jul. 2012.

[64] A. Fedorov, R. Beichel, J. Kalpathy-Cramer, J. Finet, J.-C. FillionRobin, S. Pujol, C. Bauer, D. Jennings, F. Fennessy, M. Sonka, J. Buatti, S. Aylward, J. V. Miller, S. Pieper, and R. Kikinis, "3D Slicer as an Image Computing Platform for the Quantitative Imaging Network," Magnetic resonance imaging, vol. 30, no. 9, pp. 1323-1341, Nov. 2012.

[65] Q. I. B. Alliance, DCE MRI Quantification Profile Version 1.0, Jul. 2012 .

[66] Y. Shen, F. L. Goerner, C. Snyder, J. N. Morelli, D. Hao, D. Hu, X. Li, and V. M. Runge, "T1 Relaxivities of Gadolinium-Based Magnetic Resonance Contrast Agents in Human Whole Blood at 1.5, 3, and 7 T:" en, Investigative Radiology, vol. 50, no. 5, pp. 330-338, May 2015. 
[67] M. Rohrer, H. Bauer, J. Mintorovitch, M. Requardt, and H.-J. Weinmann, "Comparison of magnetic properties of MRI contrast media solutions at different magnetic field strengths," eng, Investigative Radiology, vol. 40, no. 11, pp. 715-724, Nov. 2005.

[68] I. M. Noebauer-Huhmann, P. Szomolanyi, V. Juras, O. Kraff, M. E. Ladd, and S. Trattnig, "Gadolinium-based magnetic resonance contrast agents at 7 Tesla: In vitro T1 relaxivities in human blood plasma," eng, Investigative Radiology, vol. 45, no. 9, pp. 554-558, Sep. 2010.

[69] P. Szomolanyi, M. Rohrer, T. Frenzel, I. M. Noebauer-Huhmann, G. Jost, J. Endrikat, S. Trattnig, and H. Pietsch, "Comparison of the Relaxivities of Macrocyclic Gadolinium-Based Contrast Agents in Human Plasma at 1.5, 3, and 7 T, and Blood at 3 T," en-US, Investigative Radiology, vol. 54, no. 9, pp. 559-564, Sep. 2019.

[70] P. S. Tofts and A. G. Kermode, "Measurement of the blood-brain barrier permeability and leakage space using dynamic MR imaging. 1. Fundamental concepts," en, Magnetic Resonance in Medicine, vol. 17, no. 2, pp. 357-367, 1991.

[71] L. Østergaard, "Principles of cerebral perfusion imaging by bolus tracking," en, Journal of Magnetic Resonance Imaging, vol. 22, no. 6, pp. 710$717,2005$.

[72] M. Ibaraki, H. Ito, E. Shimosegawa, H. Toyoshima, K. Ishigame, K. Takahashi, I. Kanno, and S. Miura, "Cerebral vascular mean transit time in healthy humans: A comparative study with PET and dynamic susceptibility contrast-enhanced MRI," eng, Journal of Cerebral Blood Flow and Metabolism: Official Journal of the International Society of Cerebral Blood Flow and Metabolism, vol. 27, no. 2, pp. 404-413, Feb. 2007.

[73] N. Just, H. Adriaensen, P.-M. Chevillard, M. Batailler, J.-P. Dubois, and M. Migaud, "The sheep brain: An interesting translational model 
for functional MRI studies?" en, bioRxiv, p. 2020.09.02.280024, Sep. 2020 .

[74] H. B. Larsson, M. Stubgaard, J. L. Frederiksen, M. Jensen, O. Henriksen, and O. B. Paulson, "Quantitation of blood-brain barrier defect by magnetic resonance imaging and gadolinium-DTPA in patients with multiple sclerosis and brain tumors," eng, Magnetic Resonance in Medicine, vol. 16, no. 1, pp. 117-131, Oct. 1990.

[75] C. S. Patlak, R. G. Blasberg, and J. D. Fenstermacher, "Graphical evaluation of blood-to-brain transfer constants from multiple-time uptake data," eng, Journal of Cerebral Blood Flow and Metabolism: Official Journal of the International Society of Cerebral Blood Flow and Metabolism, vol. 3, no. 1, pp. 1-7, Mar. 1983.

[76] H. Chen, F. Li, X. Zhao, C. Yuan, B. Rutt, and W. S. Kerwin, "Extended graphical model for analysis of dynamic contrast-enhanced MRI," en, Magnetic Resonance in Medicine, vol. 66, no. 3, pp. 868-878, 2011.

[77] P. Virtanen, R. Gommers, T. E. Oliphant, M. Haberland, T. Reddy, D. Cournapeau, E. Burovski, P. Peterson, W. Weckesser, J. Bright, S. J. van der Walt, M. Brett, J. Wilson, K. J. Millman, N. Mayorov, A. R. J. Nelson, E. Jones, R. Kern, E. Larson, C. J. Carey, I. Polat, Y. Feng, E. W. Moore, J. VanderPlas, D. Laxalde, J. Perktold, R. Cimrman, I. Henriksen, E. A. Quintero, C. R. Harris, A. M. Archibald, A. H. Ribeiro, F. Pedregosa, P. van Mulbregt, and SciPy 1.0 Contributors, "SciPy 1.0: Fundamental Algorithms for Scientific Computing in Python," Nature Methods, vol. 17, pp. 261-272, 2020.

[78] R. H. Byrd, P. Lu, J. Nocedal, and C. Zhu, "A limited memory algorithm for bound constrained optimization," English, SIAM Journal on Scientific Computing, vol. 16, pp. 1190-1208, Sep. 1995.

[79] S. Butterworth, "On the Theory of Filter Amplifiers," Experimental Wireless and the Wireless Engineer, vol. 7, pp. 536-541, 1930.

[80] D. Barboriak, Quantitative Imaging Biomarkers Alliance (QIBA), 
[81] S. M. Kay, Fundamentals of Statistical Signal Processing. Prentice-Hall, 1993, vol. 1: Estimation Theory.

[82] G. J. M. Parker, C. Roberts, A. Macdonald, G. A. Buonaccorsi, S. Cheung, D. L. Buckley, A. Jackson, Y. Watson, K. Davies, and G. C. Jayson, "Experimentally-derived functional form for a populationaveraged high-temporal-resolution arterial input function for dynamic contrast-enhanced MRI," en, Magnetic Resonance in Medicine, vol. 56, no. 5, pp. 993-1000, 2006.

[83] J. U. Fluckiger, M. C. Schabel, and E. V. R. DiBella, "Model-based blind estimation of kinetic parameters in dynamic contrast enhanced (DCE)MRI," en, Magnetic Resonance in Medicine, vol. 62, no. 6, pp. 14771486, 2009.

[84] E. Butterworth, B. E. Jardine, G. M. Raymond, M. L. Neal, and J. B. Bassingthwaighte, "JSim, an open-source modeling system for data analysis," en, F1000Research, vol. 2, p. 288, Jul. 2014.

[85] C. Roberts, R. Little, Y. Watson, S. Zhao, D. L. Buckley, and G. J. M. Parker, "The effect of blood inflow and B1-field inhomogeneity on measurement of the arterial input function in axial 3D spoiled gradient echo dynamic contrast-enhanced MRI," en, Magnetic Resonance in Medicine, vol. 65, no. 1, pp. 108-119, 2011.

[86] M. H. Wilhelmi, A. Tiede, O. E. Teebken, T. Bisdas, A. Haverich, and R. Mischke, "Ovine Blood: Establishment of a List of Reference Values Relevant for Blood Coagulation in Sheep," en-US, ASAIO Journal, vol. 58, no. 1, pp. 79-82, Feb. 2012.

[87] M. C. Schabel and D. L. Parker, "Uncertainty and bias in contrast concentration measurements using spoiled gradient echo pulse sequences," Physics in medicine and biology, vol. 53, no. 9, pp. 2345-2373, May 2008 . 
[88] R. Tabbara, A. Connelly, and F. Calamante, "Multi-stage automated local arterial input function selection in perfusion MRI," en, Magnetic Resonance Materials in Physics, Biology and Medicine, vol. 33, no. 3, pp. 357-365, Jun. 2020.

[89] P. A. Yushkevich, J. Piven, H. Cody Hazlett, R. Gimpel Smith, S. Ho, J. C. Gee, and G. Gerig, "User-guided 3D active contour segmentation of anatomical structures: Significantly improved efficiency and reliability," Neuroimage, vol. 31, no. 3, pp. 1116-1128, 2006. 